\title{
A Goal Oriented Approach for Modeling and Analyzing Security Trade-Offs with Knowledge Support
}

\author{
By
}

\section{Golnaz Elahi}

A thesis submitted in conformity with the requirement for the degree of

Master of Science, 2007

Department of Computer Sciences

University of Toronto

CCopyright by Golnaz Elahi (2007) 


\begin{abstract}
A Goal Oriented Approach for Modeling and Analyzing Security Trade-Offs with Knowledge Support

Golnaz Elahi

A thesis submitted in conformity with the requirements for the degree of Master of Science, 2007

Department of Computer Science, University of Toronto
\end{abstract}

In designing software systems, security is typically only one design objective among many. It may compete with other objectives such as functionality, usability, and performance. Too often, security mechanisms such as firewalls, access control, or encryption are adopted without explicit recognition of competing design objectives and their origins in stakeholder interests. Recently, there is increasing acknowledgement that security is ultimately about trade-offs. One can only aim for "good enough" security, given the competing demands from many parties. Furthermore, one of the main challenges that software designers face is the lack of a common accessible body of security trade-offs knowledge.

This work proposes a goal oriented conceptual modeling technique for explicit and systematic modeling and analyzing security trade-offs, taking advantage of $i^{*}$ framework as the basis of the modeling notation. The technique is accompanied by a proposal for a software security trade-off knowledge base which catalogues common vulnerabilities and attacks, alternative security solutions for each one, impact of mechanisms on other goals and threats. The proposal is illustrated by several examples and case studies. 


\section{Acknowledgements}

I am in debt to my supervisor, Prof. Eric Yu, for introducing me to the research area of goal-oriented modeling, and for helping me to define and refine my ideas on topic of security trade-offs modeling and analyzing. I am grateful for knowledge and experience gained through the collaborations I had with him in the course of my Master's thesis and a joint publication which was the result of this work.

My special thanks go to John Mylopoulos for providing constructive feedbacks on preliminary ideas of this work during the "Conceptual Modeling" course project. I am grateful for knowledge gained in "Empirical Research Methods in Software Engineering" course taught by Prof. Steve Easterbrook which helped me to refine my ideas about required empirical evaluations on this work. Thank you to Jennifer Horkoff for helping me through understanding the $\mathrm{i}^{*}$ framework and modeling notation. I would like to thank Debby Repka for reading and correcting parts of my work during the "English Writing" course. And finally, in the course of this work I gratefully acknowledge the financial support received from Bell University Labs.

And on a personal note, I am in debt to my parents for their support of my education and migration to Canada. My special thanks to Masoud, my brother whose encourage and guides always help me in my personal and professional life. I am grateful for support and encourage of Amir, my best friend who during the last year I lived in Canada, called me from home hundreds times.

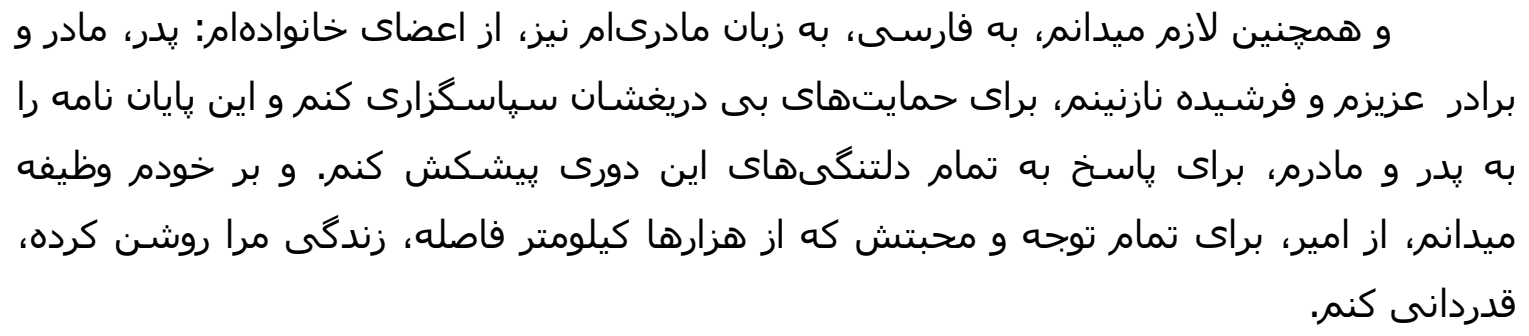




\section{Table of Contents}

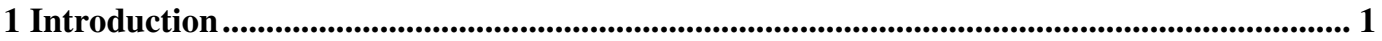

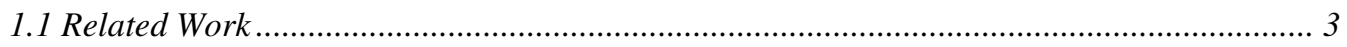

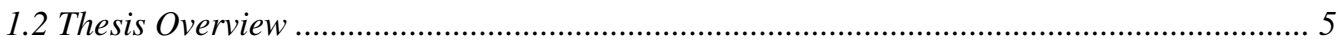

2 Existing Approaches to Modeling and Analyzing Security Trade-offs ....................................... 7

2.1 Conceptual Modeling Criteria for Security Trade-offs Analysis.......................................... 7

2.2 Existing Approaches to Security Trade-off Analysis ........................................................ 10

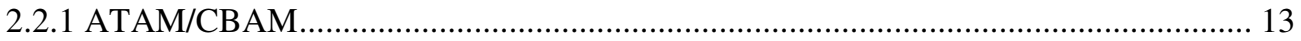

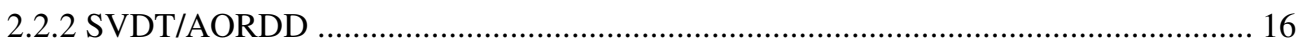

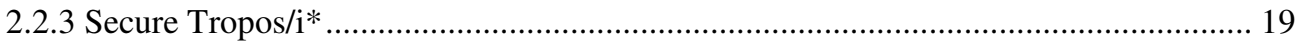

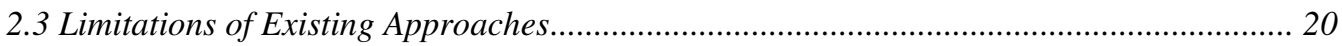

3 The Goal-Oriented Security Trade-offs Modeling and Analysis Approach........................... 25

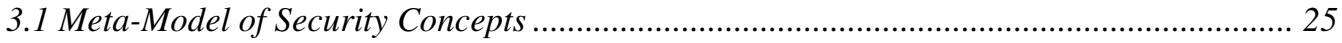

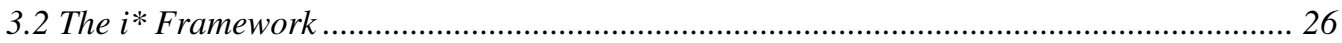

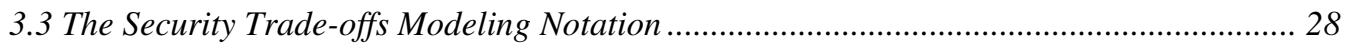

3.3.1 Malicious Actor, Goals and Tasks ........................................................................... 28

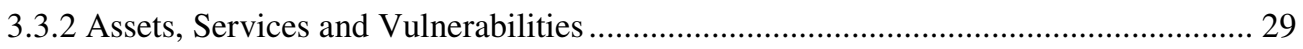

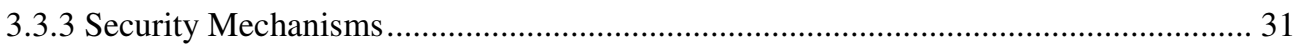

3.3.4 Relation between Attacks and Security Mechanisms .................................................. 31

3.3.5 Expressing Trade-offs by the Proposed Conceptual Structure..................................... 32

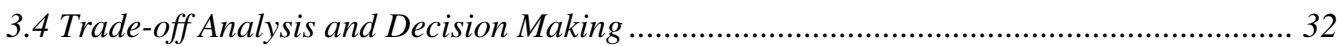

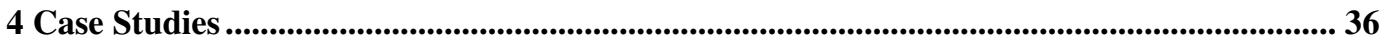

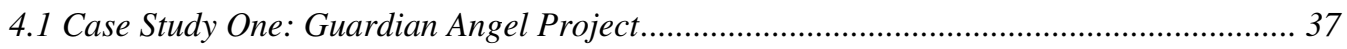

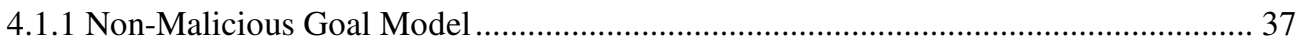




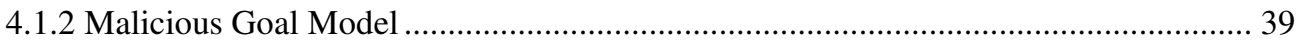

4.1.3 Security Goal Model and Trade-off Analysis ............................................................. 42

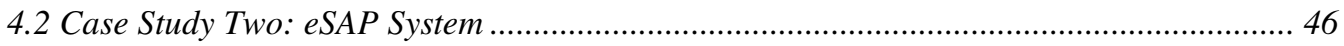

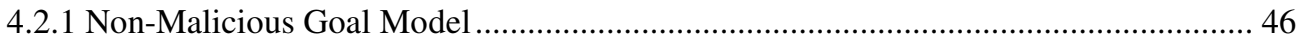

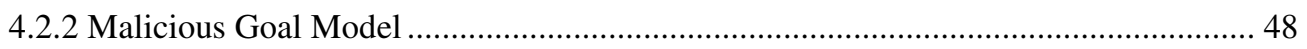

4.2.3 Security Goal Model and Trade-off Analysis ........................................................... 50

4.3 Case Study Three: Course Registration System ……………………………................ 54

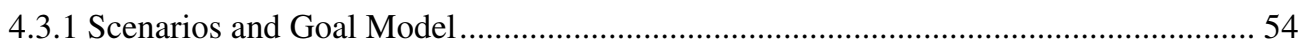

4.3.2 Tactics, Security Goals and Trade-off Analysis ........................................................ 58

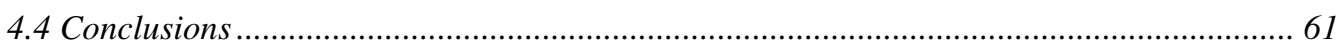

5 The Software Security Trade-offs Knowledge Base ................................................................63

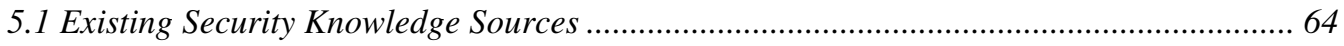

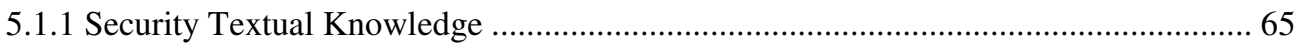

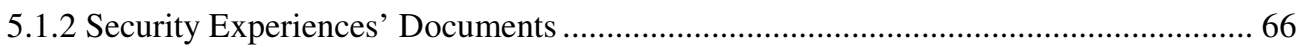

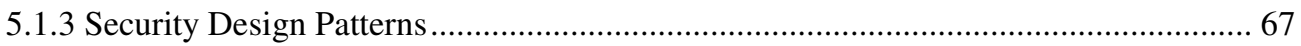

5.1.4 Structured Catalogues and Knowledge Bases........................................................... 69

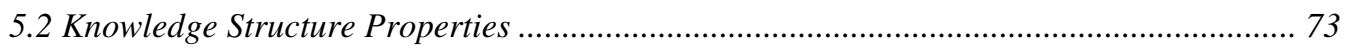

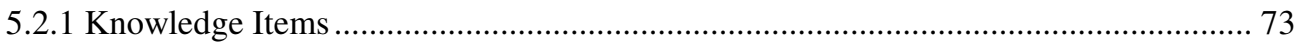

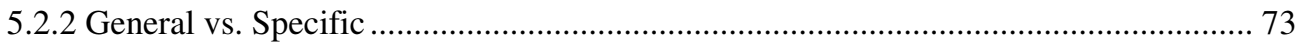

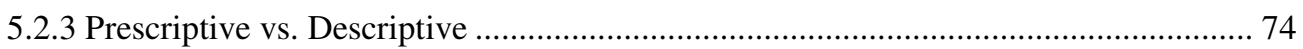

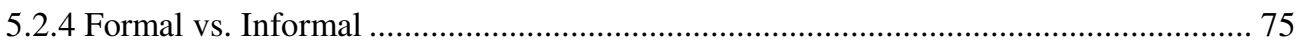

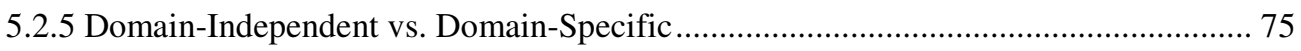

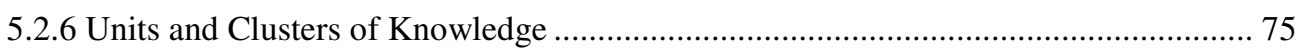

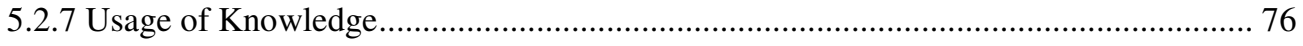

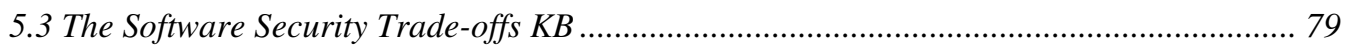

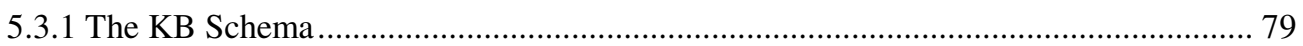




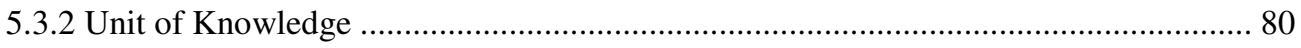

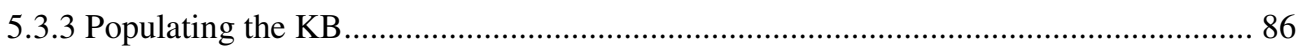

6 Example Cases of Populating the Knowledge Base........................................................................ 89

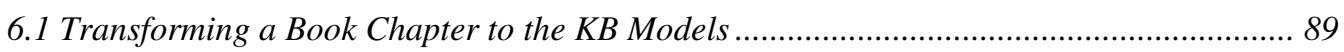

6.1.1 Extracting Knowledge Units from the Knowledge Chunk ....................................... 95

6.2 Mapping Security Design Patterns to the KB Models ........................................................ 96

6.2.1 Extracting Knowledge Units from the Knowledge Chunk ........................................ 101

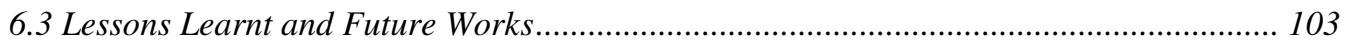

7 Contributions, Conclusions, Limitation, and Future Works....................................................... 105

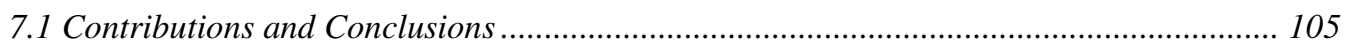

7.2 Limitation and Future Works .................................................................................. 110

7.2.1 Further Case Studies for Evaluating the Modeling Notation and Analysis Method .. 111

7.2.2 The Need for further Empirical Studies on the Proposed Trade-off Analysis

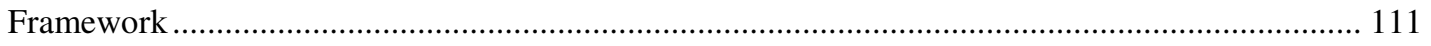

7.2.3 Further Studies on the Proposed KB.................................................................... 112

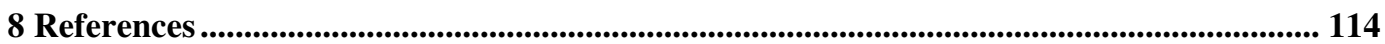




\section{List of Figures}

Figure 2-1. Meta-model of trade-off elements in ATAM. Quality Scenarios expresses the NFRs, and tactics are architectural solution.

Figure 2-2. CBAM takes business goals, cost, and quality attributes into account to calculate benefic

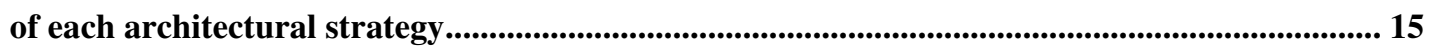

Figure 2-3. Top-level BBN topology of SVDT approach for trade-off analysis ....................................... 17

Figure 2-4. Main concepts and their relation in ARODD risk assessment [28] ....................................... 18

Figure 2-5. Overview of trade-off analysis in SVDT [6] .................................................................. 19

Figure 2-6. Part of Tropos meta-model for goals and related concepts [29] ........................................... 20

Figure 3-1. Meta-model of security concepts used in proposed modeling notation. The highlighted elements are taken from $i^{*}$ meta-model directly....................................................................... 26

Figure 3-2. Summary of $i^{*}$ modeling elements.................................................................................. 27

Figure 3-3. Example of a multi-actor system modeled using the proposed notation. Red lines (in electronic copy) do not bear different semantic, and are used to facilitate browsing the model 29

Figure 3-4. Security trade-off analysis procedure

Figure 4-1. A part of dependency relationships in the GA system, original model is obtained from 38

Figure 4-2. Detailed refinement of GA-PDA actor's goals. The model enables the designer to relate attackers and security goals to all refined GA-PDA goals and tasks. 38

Figure 4-3. A part of dependency vulnerability analysis of the GA system, original model from [5]. Each dependency is examined as a potential threat against the system. Each actor plays role of a potential attacker as well as its regular role

Figure 4-4. Attack and threats identification, original model from [22]. Malicious tasks and affected goals are located inside the boundary of the malicious actor. Malicious goals and tasks are not distinguished from regular goals 40 
Figure 4-5. Malicious actor behavior, modeled using the proposed notation. Malicious actor goals are refined

Figure 4-6. Countermeasure analysis, original model from [22]. The hypothetical threats are presented as beliefs, since their existence is based on the designer's assumption. Countermeasures contributions to the attacks are modeled, but it is not specified which actor performs the security mechanisms and what are the consequences

Figure 4-7. Security trade-off model, using the proposed extensions. Contribution of malicious goals and security goals are specified.

Figure 4-8. Security trade-offs analysis, using the proposed evaluation, using the proposed evaluation. The evaluation method steps are specified.

Figure 4-9. Security trade-offs analysis, using the proposed evaluation method 45

Figure 4-10. Stakeholders of the eSAP system, original model from [26]. Security constraints are placed between the dependencies to assign security requirements to the actors.

Figure 4-11. The professional actor goal model. The model includes Professional actor goals, and is similar to model in Figure 4-14. 48

Figure 4-12. A part of the "security diagram" for the eSAP system, original model from [26]. The model does not specify which actors have the privacy goal, how the threats work against the privacy, who threaten privacy and why

Figure 4-13. Malicious actor behavior and its relation to professional actor goals, modeled using the proposed notation. 50

Figure 4-14. Professional actor analysis, original model from [26]. The model lacks considering impact of security alternatives on other goals and threats. The impact of threats on goals (security goals and general goals) are not expressed in the models. 51

Figure 4-15. Interaction of Attacker, professional and ESAP actors. Security goals and trade-offs, modeled using the proposed notation. 52 
Figure 4-16. Security goal model trade-off analysis, using the proposed evaluation method. Evaluation method's step are tagged on the model elements ...................................................53

Figure 4-17. ATAM utility tree for the quality requirements of Course Registration System ............ 56

Figure 4-18. Goal model for the Course Registration System ........................................................... 57

Figure 4-19. Security goals and trade-offs, modeled using the proposed notation. The impact and trade-offs of each security alternative are modeled using the contribution links........................ 58

Figure 4-20. Security trade-off analysis, using the proposed evaluation method ...................................60

Figure 5-1. Elements of security design patterns of Open Group and correlation between the

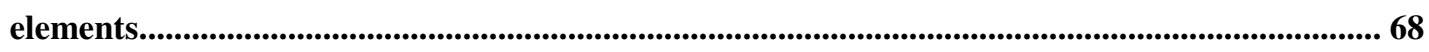

Figure 5-2. Relations between various knowledge web-portals...................................................... 71

Figure 5-3. Spectrum of generality and formality of various security knowledge sources .................. 77

Figure 5-4. The knowledge base schema. The shadowed elements are taken from $i^{*}$ notation.......... 80

Figure 5-5. Various knowledge units of the schema ....................................................................8 82

Figure 5-6. Elements of the schema which shapes the knowledge unit of Figure 5-5-A and Figure 5-5-

$\mathrm{C}$ are located in the highlighted area.. 83

Figure 5-7. A sample knowledge unit. 84

Figure 5-8. Elements of the schema which shapes the knowledge unit of Figure 5-5-B are located in the highlighted area. 85

Figure 5-9. A sample for knowledge unit of Figure 5-5-B 86

Figure 6-1. Resulting knowledge model from transforming a textbook chapter into the KB schema 92

Figure 6-2. Resulting knowledge models with highlights which indicate how textbook content are transformed to KB schema elements 94

Figure 6-3. A unit of knowledge from the whole knowledge chunk of Figure 6-2, which corresponds to the knowledge unit of Figure 5-5-C. The retrieved knowledge unit is shadowed in the knowledge chunk 95 
Figure 6-4. Mapping between elements of security design patterns and elements of the proposed KB

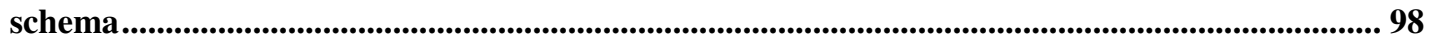

Figure 6-5. Resulting knowledge models from transforming "secure communication" design patterns to the proposed KB schema................................................................................................................................. 99

Figure 6-6. Resulting knowledge models with tags which indicate the map between security design patterns and KB schema elements. 101

Figure 6-7. A unit of knowledge from the whole knowledge chunk of Figure 6-5, which corresponds to the knowledge unit of Figure 5-5-B 102 


\section{List of Tables}

Table 2-1. A comparison of existing approaches based on the criteria of the conceptual modeling technique for security trade-off analysis............................................................................................ 24

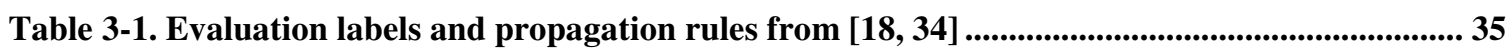

Table 4-1. Scenarios for Course Registration case study ................................................................................ 55

Table 4-2. Architectural approach analysis for Course Registration Case study ................................. 59

Table 5-1. A comparison of various knowledge sources............................................................................................. 78

Table 7-1. Summary of properties of proposed approach according to the criteria of conceptual modeling technique 107 


\section{Introduction}

"Security is about trade-offs, not absolutes."

Ravi Sandhu

Security is an increasingly significant concern for all software systems, since organizations rely on these systems, and professional by planned attacks against software systems increase every year. According to the report by the IBM Internet Security Systems X-Force research and development team [1], 7,247 new vulnerabilities were identified and recorded by the X-Force in 2006, and this number will continue to rise in 2007. Without a good enough security, organizations are not willing to share information and technology. In addition, security defects cost huge amount of time and money for many organizations.

In designing software systems, security is typically only one design objective among many. Usually, even with very good and careful design, security is known as an element of intrusiveness [2]. Security safeguards may conflict with usability, performance, and functionality. For example, there is agreement among researchers and practitioners that all efforts and security mechanisms that look secure on paper, may fail in real world because of human factors [3, 4]. If usability concerns are not addressed in the design of a secure system, users respond by circumventing security mechanisms [4, 5]. Achieving a balance between the intrusiveness of security mechanisms [2] and usability goals is an important consideration in designing successful secure software systems. 
Security goals can have their own contradictions because confidentiality, integrity, accountability, availability, and recovery from security attacks often conflict fundamentally. For example, on the one hand, the authentication process helps to maintain integrity and confidentiality. On the other hand, authentication methods such as passwords for log-in are usually barriers for users and reduce the usability and performance.

Security trade-offs are needed because of conflicts among design objectives that originate from multiple sources and stakeholders such as system's users, administrators, top managers, project managers, and customers. When some goals are not sufficiently satisfied, designers need to explore further alternatives that can better achieve those goals without detrimentally hurting others. Each potential solution can have positive effects on some goals but negative on some others. While selecting a solution among security alternatives is difficult, the more fundamental problem is that designers need to decide about alternative security mechanisms subject to multiple factors such as cost, time-tomarket, non-functional requirements (NFRs), system's functionalities, existing vulnerabilities, potential threats, security policies and standards, and individual goals of multiple stakeholders.

Ultimately, security is about balancing the trade-offs among the competing goals of multiple actors. However, typically, software designers and Information Systems (IS) professionals lack software security knowledge about common vulnerabilities and attacks for different platforms, domains and applications. In current practice, security designers often do not consider alternative solutions, or adopt security mechanisms such as firewalls, access control, or encryption without explicit recognition and systematic 
treatment of competing design objectives originating from various stakeholders. Security requirements are usually considered after the design decisions are made, and practitioners analyze security trade-offs implicitly and in their minds. This motivates questions: what techniques can help designers to express and analyze security trade-off explicitly and systematically? What conceptual modeling techniques can be used to help designers analyze security trade-offs to achieve "good enough" security?

Another challenge that designers face is the lack of a shared searchable body of security trade-offs knowledge to assist in making design trade-offs. Although there exist numerous security knowledge sources, they rarely point to security trade-offs explicitly and directly. Designers need to extract and elicit required information by browsing and interpreting the whole chunk of knowledge. For example, one may need to read an entire book or standard. Most of the security knowledge sources are unstructured text, which burdens the interpretation the knowledge. It may lead to implicit trade-off analysis because the knowledge source does not clearly indicate what trade-offs are included.

In this context, what would be a good way to organize knowledge to assist the designers for modeling and analyzing security trade-offs? What are desirable characteristics of such software security knowledge structure? And finally, what knowledge modeling technique may facilitate representing trade-offs explicitly?

\subsection{Related Work}

Trade-off analysis in general, and security trade-offs in particular have been addressed in several research works. The Architecture Tradeoff Analysis Method (ATAM) and the Cost Benefit Analysis Method (CBAM) [27] are examples of general purpose trade-off analysis frameworks which help the designer evaluate if an 
architectural decision satisfies quality scenarios and stakeholders' requirements. ATAM approach suggests a qualitative trade-off analysis, while CBAM provides a framework for calculating return of investment and quantitative trade-off analysis based on a cost benefit analysis.

Aspect-Oriented Risk-Driven Development (AORDD) [14] and Security Verification and security solution Design Trade-off analysis (SVDT) [6] provide frameworks for analyzing security trade-offs by calculating return of security investment for quantitative cost benefit analysis, by taking advantage of Bayesian Belief Networks.

Alexander suggests employing the notion of misuse cases for modeling and analyzing hostile goals and conflicts and trade-offs they impose on other use cases [49]. Johnson et al. suggest using influence diagram accompanied with Bayesian Belief Networks probabilistic analysis for analyzing the information security solutions [15].

Several goal oriented approaches propose methods for modeling and analyzing malicious and security goals, trust, conflicts and competition issues among agents. However, most of the proposed methods lack an explicit approach for modeling and analyzing security trade-offs.

This thesis proposes a goal oriented approach for modeling and analyzing malicious and security goals in addition to the ordinary goals of actors, and provides a security trade-off analysis procedure. The modeling and analyzing framework is accompanied by a security trade-off knowledge base which provides the designer with the required knowledge for trade-off modeling. The KB takes advantage of the proposed goal oriented notation for codifying the trade-offs knowledge. 


\subsection{Thesis Overview}

This work proposes a conceptual modeling technique for modeling and analyzing security trade-offs. To answer the raised questions, the criteria for a suitable conceptual modeling technique for dealing with security trade-offs are discussed. Chapter 2 provides a survey of the existing approaches to modeling and analyzing security trade-offs. This chapter discusses criteria of a conceptual modeling technique which enables the designer to model and analyze security trade-offs. A number of existing approaches in modeling and analyzing security requirements and trade-offs are overviewed, and three selected approaches for trade-off analysis are studies in detail, and are compared based on the criteria.

In Chapter 3, from analyzing the limitations of existing methods and discussed criteria, a goal-oriented notation for modeling and analyzing security trade-offs is proposed. The proposed notation is an extension to the $i^{*}$ notation, a goal oriented requirements modeling language. In this section, the $i^{*}$ framework is reviewed briefly. A set of security extensions to the $\mathrm{i}^{*}$ modeling notation is introduced using examples. A meta-model of security concepts which conations extended elements to $i^{*}$ meta-model is provided. A trade-off analysis procedure which takes advantage of goal model evaluation techniques is then presented.

In Chapter 4, we apply the notation to three example cases originally used to illustrate other approaches to security trade-offs. The resulting models are compared with the models provided in the original case studies. The main objective of the case studies is to evaluate and compare existing approaches and the proposed method in terms the 
expressiveness of the resulting models and comprehensiveness of the trade-off analysis results.

Chapter 5 gives a survey of various security knowledge sources considering and comparing properties and limitations of each one to extract a set of desired properties for a software security trade-offs Knowledge Base (KB). Based on the desired properties and the strong points of existing knowledge sources, we propose a schema for gathering and cataloguing security trade-offs knowledge in the form of a KB. The chapter concludes with a discussion on the unit of knowledge and process of populating the KB.

Chapter 6 gives two example cases of populating the KB from two different knowledge sources, a textbook chapter and a security design pattern. The aim is to examine how various knowledge formats can be transformed to the proposed KB from both a semi-structured and an unstructured body of knowledge. We conclude in Chapter 7, with a summary of contributions, limitations of the approach, and future works. 


\section{Existing Approaches to Modeling and Analyzing Security Trade-offs}

This chapter discusses criteria of a conceptual modeling technique which enables the designer to model and analyze security trade-offs. A number of existing approaches in modeling and analyzing security requirements and trade-offs are overviewed, and three selected approached of trade-off analysis are studied and compared based on the criteria.

\subsection{Conceptual Modeling Criteria for Security Trade-offs Analysis}

Trade-off analysis is a systematic examination of the advantages and disadvantages of each proposed requirements and/or design approach for a system [49] to achieve the right balance among several competing goals. When some goals are not sufficiently satisfied, designers need to explore further alternatives that can better achieve those goals without detrimentally hurting others. Each potential solution can have positive effects on some goals but negative on some others.

A careful and systematic process for security trade-off analysis can be very challenging, because a wide range of security mechanisms, solutions and frameworks need to be considered. In addition, designers need to make trade-offs between various goals of multiple stakeholders. In this context, we examine what can conceptual modeling techniques offer for analyzing security trade-offs. What are the criteria of a proper conceptual modeling technique for dealing with security trade-offs? What items need to be modeled, and how they would be used in the trade-off analysis? 
A conceptual modeling technique that expresses the security trade-offs and enables security trade-off analysis should model three kinds of concepts: i) Goals, ii) Actors and iii) Security specific concepts.

i) Goals: Security trade-offs are about conflicts among design objectives that originate from goals of various stakeholders. While selecting a solution among security alternatives is difficult, the more fundamental problem is that designers need to decide about alternatives security mechanisms subject to multiple factors such as cost, time-tomarket, existing vulnerabilities, potential threats, non-functional requirements (NFRs), system's functionalities, security policies, standards, and individual goals of various stakeholders. The stakeholders include system's users, administrators, maintainers, top managers, project managers, and customers. Therefore, the "goal" concept is a basic modeling construct required in the conceptual modeling technique for dealing with tradeoffs.

The conceptual technique should be able to model the relations among goals such as competition and contribution. One required feature of the conceptual modeling technique is to provide means for structuring the contributions of goals, expressing relations among goals, impacts of goals on each other, and the extents and measures of goals satisfaction, contribution and competition. The measures could be quantitative or qualitative. Quantitative measures can greatly simplify decision making, but the general problems with the quantitative measures are lack of agreed metrics for the measurement or unavailability of accurate measures, especially in earlier stages of development. Thus, the modeling technique should be able to support trade-off analysis despite inaccurate or incomplete knowledge about goals. 
ii) Actors: Design objectives typically come from multiple sources. The appropriate conceptual modeling technique should be able to model multiple actors that impose competing goals to the designer. It should provide means to express the relation such as dependency between actors, and means to trace back goals to the actors. Tradeoffs could occur within a single actor or across many, and the modeling technique should be able to model both cases.

iii) Security Specific Concepts: The conceptual modeling technique that enables security trade-off analysis should model security specific concepts such as threats, vulnerabilities, and safeguards. Threats can be viewed as malicious actors' goals which typically have conflicts with goals of ordinary actors. Conflicts among stakeholders' goals are usually unavoidable, and the designer needs to balance the trade-offs among conflicting goals. In contrast, sources of threats and attacks are not stakeholders, and the conflict they have with other goals must be removed or mitigated.

To decide among alternative solutions for potential threats, decision makers need a measurable expression of the security level of solutions [6]; therefore the modeling technique should provide means to model to what extent threats are successful, how attacks influence on goals, whether countermeasures control the threats, and whether the goals are at risk.

Trade-off analysis needs to be accompanied by a procedure for evaluating security alternatives. The proper trade-off analysis method should evaluate the impact of each alternative solution on goals and potential threats. It should answer to what extent the goals are satisfied or denied, threats are controlled, and vulnerabilities are patched. 
The procedure should be able to analyze the trade-offs in case of incomplete or inaccurate knowledge about goals' contributions and measures of security aspects.

The technique should satisfy general requirements for conceptual modeling as well. The purpose of conceptual modeling in software engineering is to make real world concepts and relations understandable [7]. Conceptual models reflect parts of reality that are important for performing tasks. They should facilitate communication among various stakeholders and prevent misinterpretations of requirements [8]. Therefore, in selecting or developing a conceptual modeling technique, scalability, expressiveness, complexity, and possibility for automation should be considered.

\subsection{Existing Approaches to Security Trade-off Analysis}

Several approaches have been proposed for modeling security aspects of the software systems. Some approaches employ qualitative trade-off analysis, while some techniques propose using quantitative trade-off modeling and analysis methods. A number of techniques employ goal and agent oriented approaches for expressing and analyzing security trade-offs, while some methods use object oriented notations such as UML as the modeling language. This section overviews existing approaches in this domain briefly.

The notion of "abuse case", proposed by McDermott [9], helps to model a type of interaction between a system and one or more actors where the results of the interaction are harmful to the system. Another similar approach is the notion of "misuse case" which was proposed by Sindre [50]. A misuse case is a use case from the point of view of an actor hostile which poses a threat to the system. Sindre claim that looking at systems from a misuser perspective increases the chance of discovering threats that would 
otherwise be ignored. The misuse case approach is adopted by Ian Alexander in [49] for trade-off analysis. Alexander asserts that use/misuse case representation may make tradeoff analysis and human judgments more informed and systematic by visualizing the structure of the situation accurately in a way that emphasizes the essential points of conflicts and trade-offs.

The UMLsec modeling language [10] is an example of a security-specific conceptual modeling approach for modeling security requirements and aspects of the system. The approach in [11] proposes a framework of core security requirements artefacts to describe the security requirements. The meta-model of the core artefacts includes concepts such as assets, threats, security goals, functional requirements, and security requirements. In [12], the authors identify asset of core security artefacts in a framework for security requirements elicitation and analysis.

SecureUML is a modeling language for the model-driven development of secure, distributed systems based on the Unified Modeling Language (UML). SecureUML takes advantage of Role-Based Access Control (RBAC) for specifying authorization constraints by defining a vocabulary for annotating UML-based models with information relevant to access control [13]. The SecureUML meta-model is defined as an extension of the UML meta-model, and the concepts of RBAC are represented directly as meta-model types.

The CORAS framework [14] provides another UML profile for risk assessment. The proposed profile defines UML stereotypes and rules for specialized UML diagrams for support of model-based risk assessment. Asset diagram is proposed to identify and value assets; threat diagram captures unwanted incidents causing loss in asset value. State 
analysis diagram is proposed to estimate and document the frequency and consequence of the unwanted incidents, and treatment diagrams are models extended with specialized use cases representing treatments.

In [15], probabilistic inference on security influence diagrams is used to support trade-off analysis using Bayesian networks. In this work, authors discuss the criteria of the language for security requirements modeling and analyzing. They assert that the language should be able to represent decision's maker goals, domain of control, and causal relation between goals (indirect and controlled). For managing the definitional uncertainty the language must represent what mean by concepts, and represent the causal and empirical uncertainty.

[16] proposes a risk analysis framework integrated with Requirements Engineering and Security Engineering to align other business goals and architectural constraints with security requirements where risks are at the center of the alignment of business and architectural goals.

In recent years, agent and goal oriented approaches in Requirements Engineering have emerged as new approaches to analysis and design of complex software systems. Examples of such approaches and frameworks are KAOS [17], the NFR framework [18], the $i^{*}$ framework [19], and Tropos [20]. In such approaches, systems are modeled as intentional agents collaborating or competing with each other to achieve their goals. Security issues arise when some actors, while striving to achieve their own goals, intentionally or unintentionally threaten other actors' goals. Therefore, agent and goal oriented approaches provide a suitable basis for dealing with competing goals of multiple actors. Several approaches such as $[21,22,23,24,25,26]$ propose frameworks for 
modeling and analyzing security concepts and trade-offs by taking advantage of agent and goal oriented techniques. The majority of these approaches employ qualitative tradeoff analysis, while [24] suggests a quantitative approach for analyzing security requirements.

In the remaining part of this chapter, we selected three methods of modeling and analyzing security trade-offs are reviewed. We study how well these modeling notations and trade-off analysis methods are matched with the criteria discussed in the previous section. Architecture Tradeoff Analysis Method (ATAM) and Cost Benefit Analysis Method (CBAM) [27] are studied because they provide a general purpose and widelyused trade-off analysis method which consider security as a quality property. We study the approaches in $[21,22,23,24,25,26]$ that employ agent and goals oriented modeling approaches for dealing with security trade-offs. Security Verification and security solution Design Trade-off analysis (SVDT) method employs UMLsec as the modeling language and Bayesian Belief Nets $(\mathrm{BBN})$ for quantitative analysis [6]. We study AORDD and SVDT as representative of quantitative analysis methods.

\subsubsection{ATAM/CBAM}

Bass et al. [27] introduces a framework to model quality attributes and architectural decisions by notion of scenarios and tactics respectively. A quality attribute scenario is a quality-attribute-specific requirement, and consists of six parts: Source of stimulus, Stimulus, Environment, Artifact, Response, and Response measure. Achievement of quality scenarios relies on tactics. ATAM [27] is an evaluation method to analyze whether an architecture decision satisfies particular quality goals. ATAM helps designers to evaluate alternative tactics for quality scenarios. The evaluator creates 
a "Quality Attribute Utility Tree" where utility is the expression of the overall goodness of the system. Quality requirements are children of utility, and can be refined to more detailed requirements. Scenarios form the leaves of the tree. The difficulty to achieve and importance of each scenario is ranked by a high/medium/low scheme. Then, scenarios that have at least one high priority of importance or difficulty are chosen for a detail analysis to examine if the selected tactics satisfy the scenario.

The result of analysis is an "Architectural Approach Analysis Table" for each quality scenario. In this table, evaluators identify and record sensitivity, tradeoff, risks and non-risks points for alternative tactics. Sensitivity and tradeoff points are architectural decisions that have effect on one or more quality attributes, the former positively and the latter negatively. In ATAM, a risk is defined as an architectural decision that may lead to undesirable consequences, and non risk points are defined in the opposite way. The conceptual elements related to trade-offs in ATAM may be captured in a meta-model as in Figure 2-1.

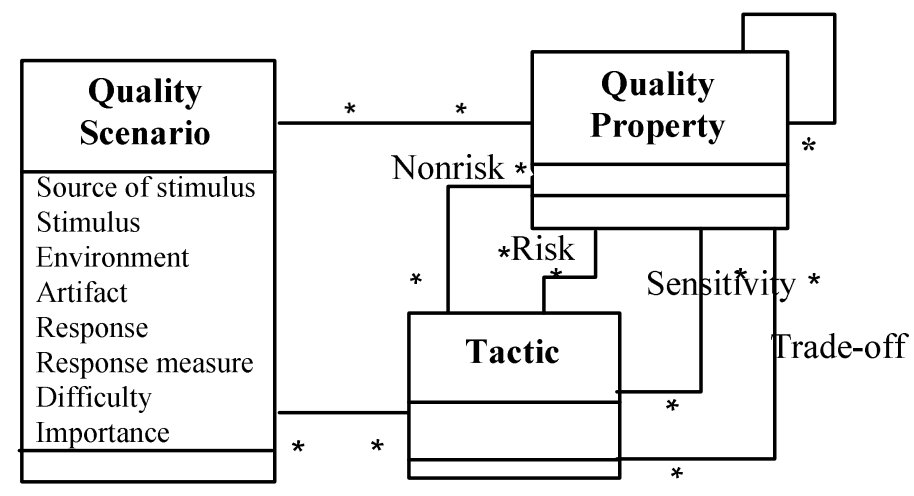

Figure 2-1. Meta-model of trade-off elements in ATAM. Quality Scenarios expresses the NFRs, and tactics are architectural solution 
However, the ATAM is missing an important consideration which is the cost factor. The CBAM begins where the ATAM concludes, and depends upon the artifacts that the ATAM produces as output. The Figure 2-2 depicts the context for the CBAM.

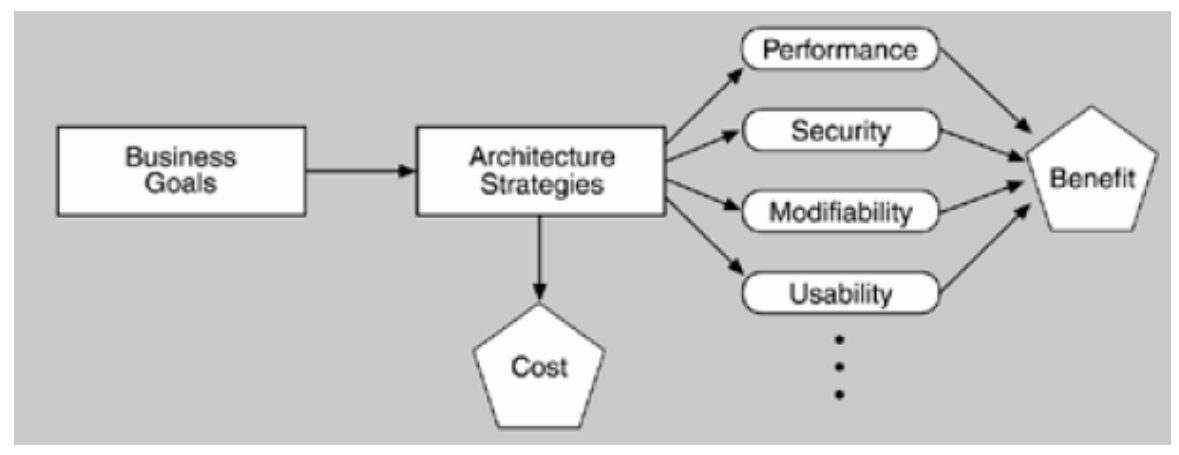

Figure 2-2. CBAM takes business goals, cost, and quality attributes into account to calculate benefic of each architectural strategy

The CBAM uses scenarios as a way to concretely express and represent specific quality attributes. The utility is based on the importance of each scenario being considered with respect to its response value. Stakeholders vote for scenarios to select the top priority scenarios for further analysis. Stakeholders assign value of stimulus-response utility for each scenario, which helps to create the utility-response curves. The utilityresponse curve depicts how the utility derived from a particular response varies as the response varies.

Designers develop architectural strategies for scenarios and determine the corresponding expected quality attribute response levels. Each architectural strategy has a cost, and affects multiple quality attributes which poses trade-offs on other quality attributes. It is important to determine the utility of these additional side effect attribute responses that arise as a result of applying the architectural strategy. CBAM takes the utility of these "side effects" into account when considering a strategy's overall utility. 
CBAM calculates overall utility of an architectural strategy across scenarios from the utility-responses by summing the utility associated with each architectural strategy.

The overall utility is combined with the project cost of an architectural strategy to calculate a final Return On Investment (RIO) measure. The ROI value for each architectural strategy is the ratio of the total benefit to the cost. Using this ROI value, the architectural strategies can be rank-ordered and compared, and final decision is made.

\subsubsection{SVDT/AORDD}

Houmb et al. [6] propose SVDT approach using UMLsec for modeling security solutions and $\mathrm{BBN}$ as the basis of trade-off analysis implementation. UMLsec is used to specify security requirements, and UMLsec tools verify if the design solutions satisfy the security requirements. Design solutions that pass the verification are then evaluated using security solution design trade-off analysis. This approach is a complementary work on Aspect-Oriented Risk-Driven Development (AORDD) framework [28]. AORDD framework provides risk assessment process and cost-benefic trade-off analysis. Both SVDT and AORDD trade-off analysis methods compute Return on Security Investment (RoSI) using BBN.

Figure 2-3 gives the top-level BBN topology for trade-off analysis. In the BBN graph, nodes correspond to events or concepts. The potential states of a node are expressed using Probability Density Functions (PDF). Pieces of information or evidence

are inserted into the leaf nodes and propagated through the network using the PDFs. A PDF describes one's confidence in the various outcomes of the node and depends conditionally on the status of the connected nodes. 


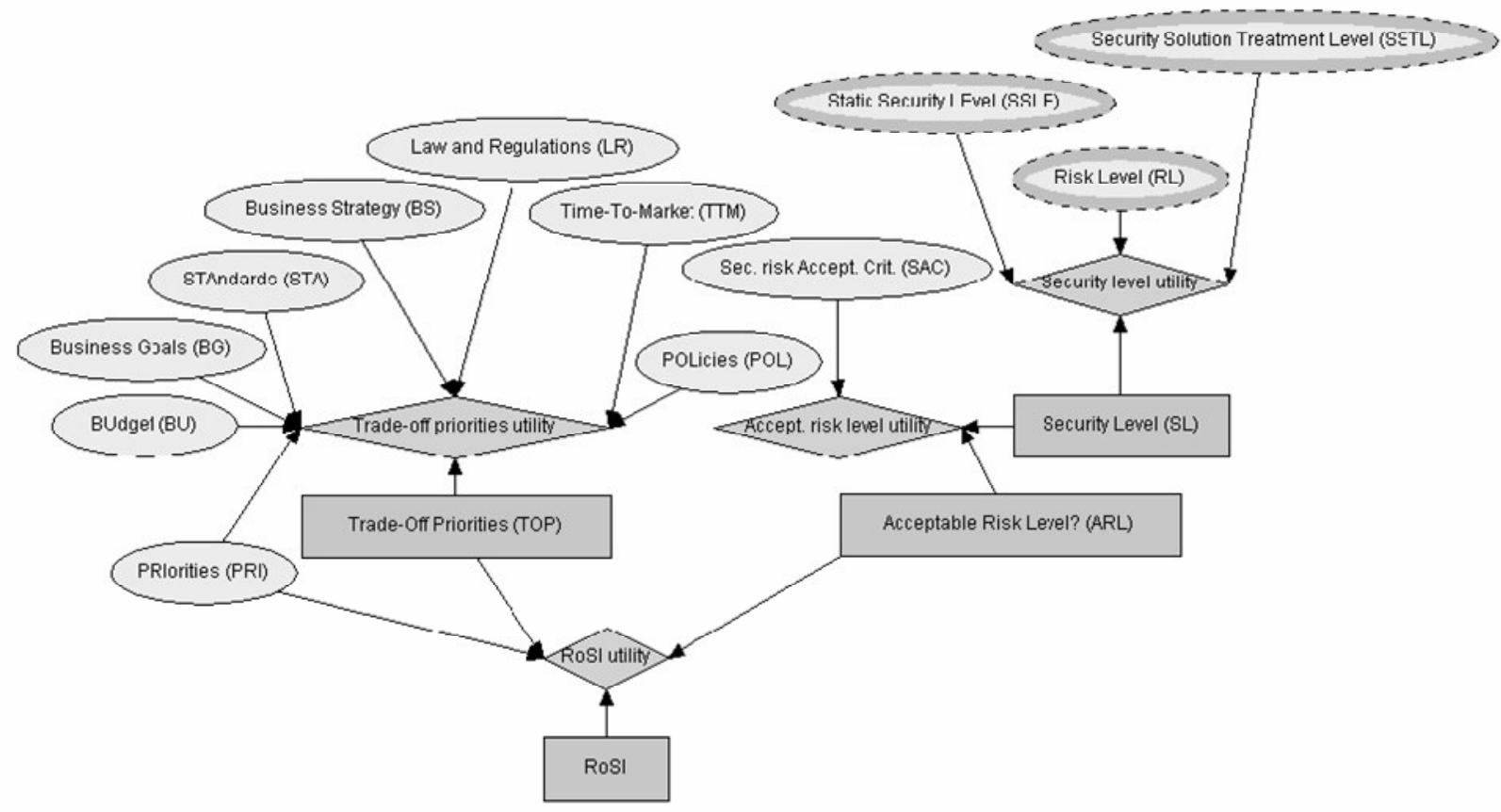

Figure 2-3. Top-level BBN topology of SVDT approach for trade-off analysis

The proposed BBN in [6] consists of four main parts: (1) trade-off variables, which are combined by the trade-off priorities utility. The trade-off variables each represent different issues that influence which security solution is best for the problem under consideration; (2) security level variables, which are combined by the security level utility; (3) security risk acceptance variables, which are combined with the security level variables by the accept risk level utility; and (4) RoSI variables, which are combined with the variables from the other parts, using the RoSI utility.

Figure 2-4 illustrates the relationship between the main concepts involved in AORDD risk assessment, which specifies the structure of the inputs to the AORDD costbenefit trade-off analysis. The result of risk assessment is a list of misuses which need security treatments. This list and a list of alternative security treatments are fed into the BBN to compute the RoSI. 


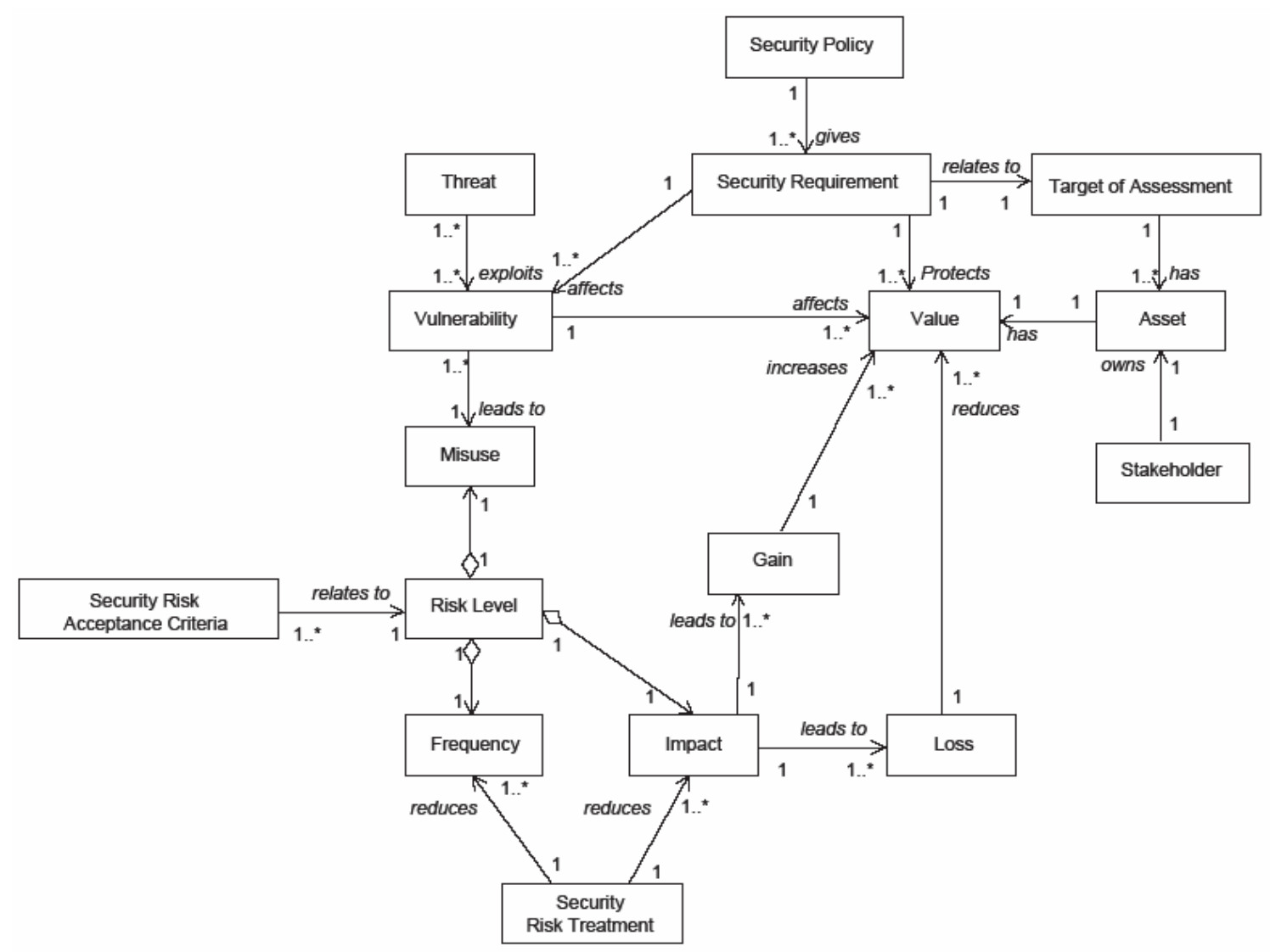

Figure 2-4. Main concepts and their relation in ARODD risk assessment [28]

RoSI is computed using a set of trade-off parameters, such as priorities, security risk acceptance criteria, standards, laws and regulations, and in particular, business goals, budget, TTM and policies. RoSI for a particular solution is derived by evaluating the effect and cost of the solution against the security requirements, or the misuse impact and frequency, if the solution is intended to treat a misuse [6].

Figure 2-5 gives an overview of the trade-off analysis parameters in SVDT. The parameters on the left side of the figure are "input parameters, and are the information that is traded off". The parameters on the right side are "trade-off parameters, meaning the information that is used to trade-off the input parameters." For each misuse, designers 
suggest a number of alternative verified security solutions. Generally, trade-off parameters are fixed constraints which have no semantic relation to security solutions and misuses.

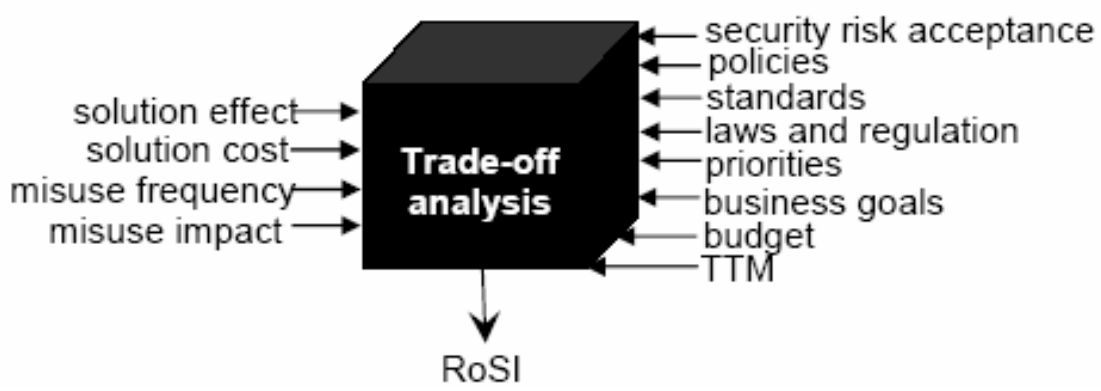

Figure 2-5. Overview of trade-off analysis in SVDT [6]

\subsubsection{Secure Tropos/i*}

The proposed approaches in $[21,22,23,25,26]$ take advantage of the $i^{*}$ and Tropos frameworks. In [21, 22], security is treated as an issue arising among social agents. The approach in [21] suggests using relationships among strategic actors for analyzing security requirements. In [21], potential attackers of the systems are distinguished from other actors of the system. [22] proposes a methodological framework for dealing with security requirements based on the $i^{*}$ notation. In [23], a framework known as Secure Tropos for modeling and analyzing security requirements based on notions of trust, ownership, and permission delegation is developed.

In $[25,26]$, the "threat" and "security constraint" modeling elements are added to the $i^{*}$ meta-model. They suggest employing threat elements in the "security diagram" to express potential violation against the security goals. The security constraints are used to impose security requirements on actors' dependencies. The meta-model of related 
concepts to the Tropos goal model, which is the core of all these approaches, is depicted in Figure 2-6.

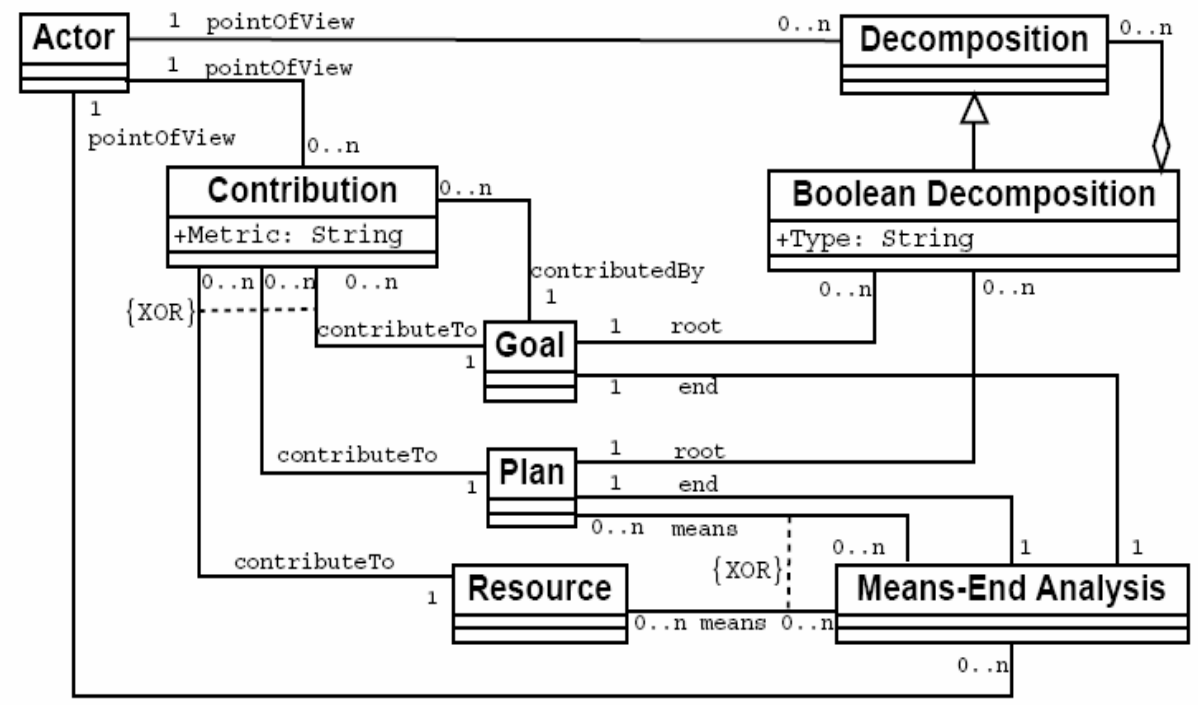

Figure 2-6. Part of Tropos meta-model for goals and related concepts [29]

\subsection{Limitations of Existing Approaches}

In ATAM approach, trade-offs among quality scenarios and tactics in the "Architectural Approach Analysis" table are expressed indirectly and implicitly, because trade-off and risk points, instead of referring to quality scenarios, refer to the quality properties which are affected by the tactic. Hence, the resulting tables do not capture the trade-offs among various tactics and design objectives. Moreover, ATAM does not incorporate the degree of sensitivity or trade-off between tactics and qualities in the analysis procedure, because "Architectural Approach Analysis" table does not provide means for expressing the impact of tactics on stimuli (attacks), and does not specify the consequences of applying a solution on objective of other quality scenarios.

ATAM lacks considering the impact of each tactic on stimuli of security scenarios (attacks). The impact of tactics on quality attributes are not captured qualitatively or 
quantitatively. Finally, the framework of scenarios, tactics and ATAM method does not provide means to model and analyze security concepts such as threat, vulnerability, and asset specifically. Besides, ATAM does not consider cost as a trade-off factor that usually has the highest priority over the other factors. CBAM is proposed to address this weakness.

However, the CBAM approach has limitations in expressing and analyzing security trade-offs. First of all, the CBAM approach to trade-off analysis requires the designer obtain accurate quantitative measures of current response and expected response of architectural strategies for each affected scenarios. The major issues are unavailability and inaccuracy of such measures in the early stages of development, and lack of agreement on metrics and measurement methods.

Once the impact of each strategy is recognized, the utility of each strategy is determined. CBAM relies on the utility values gathered from votes of various stakeholders through consensus. In this way, CBAM resolves the conflict of goals among stakeholders by computing the ROI of each strategy based on their votes for utility of each strategy. However, the accuracy and reliability of the utility values and stakeholders' confidence in their votes is an issue in validity of CBAM analysis approach. There are group of stakeholder that may not have the expertise to evaluate the utility of various architectural strategies based on the current and expected responses of strategies, and CBAM does not distinguish non-expert stakeholders' votes in the trade-off analysis process. 
SVDT and AORDD rely on quantitative computation and probabilistic inference for trade-off analysis. This requires the software designers obtain quantitative measures of the impact of misuses and solutions. The major limitation is the inaccuracy or unavailability of quantitative data on the impact of misuses and solutions especially in the early stages of the development lifecycle.

Generally, the suggested BBN topologies in SVDT and AORDD do not consider a more general source of trade-off inputs such as NFRs and functionalities, and the tradeoff inputs to the designed BBN are limited to factors such as budget, laws and regulation. Besides, the AORDD meta-model of risk assessment concepts (Figure 2-5) does not consider the relation between "security risk treatment" and other entities such as "security requirement", "threat", and "vulnerability". The AORDD meta-model would be lacks considering more general concepts such as goals, other quality requirements, and actors. Moreover, the trade-off inputs and information are given to a $\mathrm{BBN}$, and the final RoSI is computed automatically, which makes the analysis efficient. Since, the relationships between the various states of the variables are specified in terms of the node probability matrix in $\mathrm{BBN}$, this automatic trade-off analysis process can be traced by the designer. However, it may be difficult for the designer to follow what aspects of the design caused the difference in the final results.

The result of the trade-off analysis is finally a number indicating the return of investment for each security solution, and making the decision among security mechanisms is based on the cost-benefit analysis of each security solution. However, the RoSI value lacks considering some trade-off factors during decision makings, because impact of the security mechanisms on other design objectives such as functionality and 
performance is not considered in RoSI. The single value of RoSI does not indicate what trade-offs designers are making. Finally, SVDT and AORDD employ UML-based notations such as UMLSec for specifying security requirements and solutions. UMLsec is a rich notation for modeling security solutions, but it inherits weaknesses of UML in modeling design objectives from multiple actors' viewpoints.

Agent and goal oriented approaches provide a proper conceptual structure for modeling multiple actors, goals, softgoals and goals' refinements and dependencies. Although approaches in $[21,22,23,25,26]$ have the conceptual basis for security tradeoff modeling and analysis, a mechanism for security trade-offs analysis is not elaborated in these frameworks. The method in [22] lacks a direct and explicit way to model the competition among malicious and non-malicious actors' goals. Moreover, trade-off modeling among goals is limited to the non-malicious actors. The proposed framework in [23] does not support modeling security concepts such as malicious behavior.

In $[25,26]$, threats are modeled in the security diagram, but the model does not trace security goals or threats to the source actors. This approach does not consider modeling the relation between countermeasures and attacks either. Generally, the existing goal oriented approaches do not model security trade-offs explicitly; therefore, security trade-off analysis is not systematically organized.

Table 1 summarizes a comparison of the studied approaches based on the evaluation criteria. 
Table 2-1. A comparison of existing approaches based on the criteria of the conceptual modeling technique for security trade-off analysis

\begin{tabular}{|c|c|c|c|}
\hline $\begin{array}{c}\text { Method } \\
\text { Requirement }\end{array}$ & ATAM/CBAM & SVDT/AORDD & i*/Tropos \\
\hline Goals & Expressed in terms of scenarios & $\begin{array}{c}\text { Limited to security } \\
\text { requirements and } \\
\text { fixed BBN parameters }\end{array}$ & Explicit goals \\
\hline Relations of goals & Not model explicitly & $\begin{array}{c}\text { Limited to UMLsec } \\
\text { models }\end{array}$ & $\begin{array}{c}\text { Modeled using } \\
\text { contribution links }\end{array}$ \\
\hline $\begin{array}{c}\text { Extents of goal } \\
\text { satisfaction }\end{array}$ & $\begin{array}{c}\text { Not expressed in ATAM/ } \\
\text { Expressed in terms of Utility- } \\
\text { Response Curves in CBAM }\end{array}$ & Quantitatively & Qualitatively \\
\hline $\begin{array}{c}\text { Inaccurate or } \\
\text { incomplete } \\
\text { knowledge }\end{array}$ & Not modeled & $\begin{array}{c}\text { In terms of probability } \\
\text { density functions (pdf) } \\
\text { in BBN }\end{array}$ & $\begin{array}{c}\text { In terms of } \\
\text { unknown } \\
\text { contribution }\end{array}$ \\
\hline $\begin{array}{c}\text { Goals contribution } \\
\text { structure }\end{array}$ & $\begin{array}{c}\text { Utility tree in ATAM doesn't } \\
\text { capture the contributions of } \\
\text { scenarios/ CBAM does not model }\end{array}$ & $\begin{array}{c}\text { Not modeled } \\
\text { Expressed implicitly by multiple } \\
\text { stimuli sources in ATAM/ in } \\
\text { CBAM multiple stakeholders } \\
\text { votes are taken into account }\end{array}$ & Not modeled \\
\hline $\begin{array}{c}\text { Multiple actors } \\
\text { contribution links }\end{array}$ \\
\hline $\begin{array}{c}\text { Trade-off within a } \\
\text { single actor or } \\
\text { across actors }\end{array}$ & $\begin{array}{c}\text { Single actor in ATAM/ multiple } \\
\text { actors in CBAM }\end{array}$ & $\begin{array}{c}\text { Modeled in terms of } \\
\text { agents/actors/ roles/ } \\
\text { positions }\end{array}$ \\
\hline $\begin{array}{c}\text { Security Specific } \\
\text { Trade-off Concepts }\end{array}$ & Not modeled & Some concepts are \\
modeled & $\begin{array}{c}\text { Some concepts are } \\
\text { modeled }\end{array}$ \\
\hline $\begin{array}{c}\text { Trade-off analysis } \\
\text { method }\end{array}$ & $\begin{array}{c}\text { Qualitative analysis in ATAM/ } \\
\text { Quantitative analysis in CBAM }\end{array}$ & Quantitative analysis & $\begin{array}{c}\text { Qualitative and } \\
\text { quantitative analysis }\end{array}$ \\
\hline
\end{tabular}




\section{The Goal-Oriented Security Trade-offs Modeling and Analysis Approach}

In this chapter, a goal-oriented notation for modeling and analyzing security trade-offs is proposed. The proposed notation is an extension on the $\mathrm{i}^{*}$ notation, a goaloriented requirements modeling language. In this part, the $i^{*}$ framework is introduced briefly, and security extensions to the $\mathrm{i}^{*}$ modeling notation are described using examples. The notation is accompanied with a trade-off analysis procedure. (Portion of an earlier version of this chapter appears in [40])

\subsection{Meta-Model of Security Concepts}

We propose a meta-model of security concepts for systematically addressing security trade-offs (Figure 3-1). The core of the meta-model is the concepts of goals and actors derived from the criteria of the conceptual modeling technique that enables security trade-offs analysis. The meta-model is developed by considering the limitations of existing approaches to security trade-off modeling, and reviewing well known security knowledge sources such as NIST's guidelines and standards [30], CERT [31], and widely used textbooks [32, 33].

The core of this schema is goals and actors. Security mechanisms and security goal are specialization of mechanisms and goals. Security mechanisms prevent or detect an attack, or recover the system from attacks and threats. Target of security threats and attacks is an $\operatorname{asset}(s)$. Actors are interested in assets, or they own assets, or the actor has delegated the usage permission of the assets to the other actors [23]. The attacks usually occur through a weakness or a backdoor to the system which is known as vulnerability. 
Attacks might be unintentional, or result of an accident or a human error. Natural disasters are another type of threats against the systems.

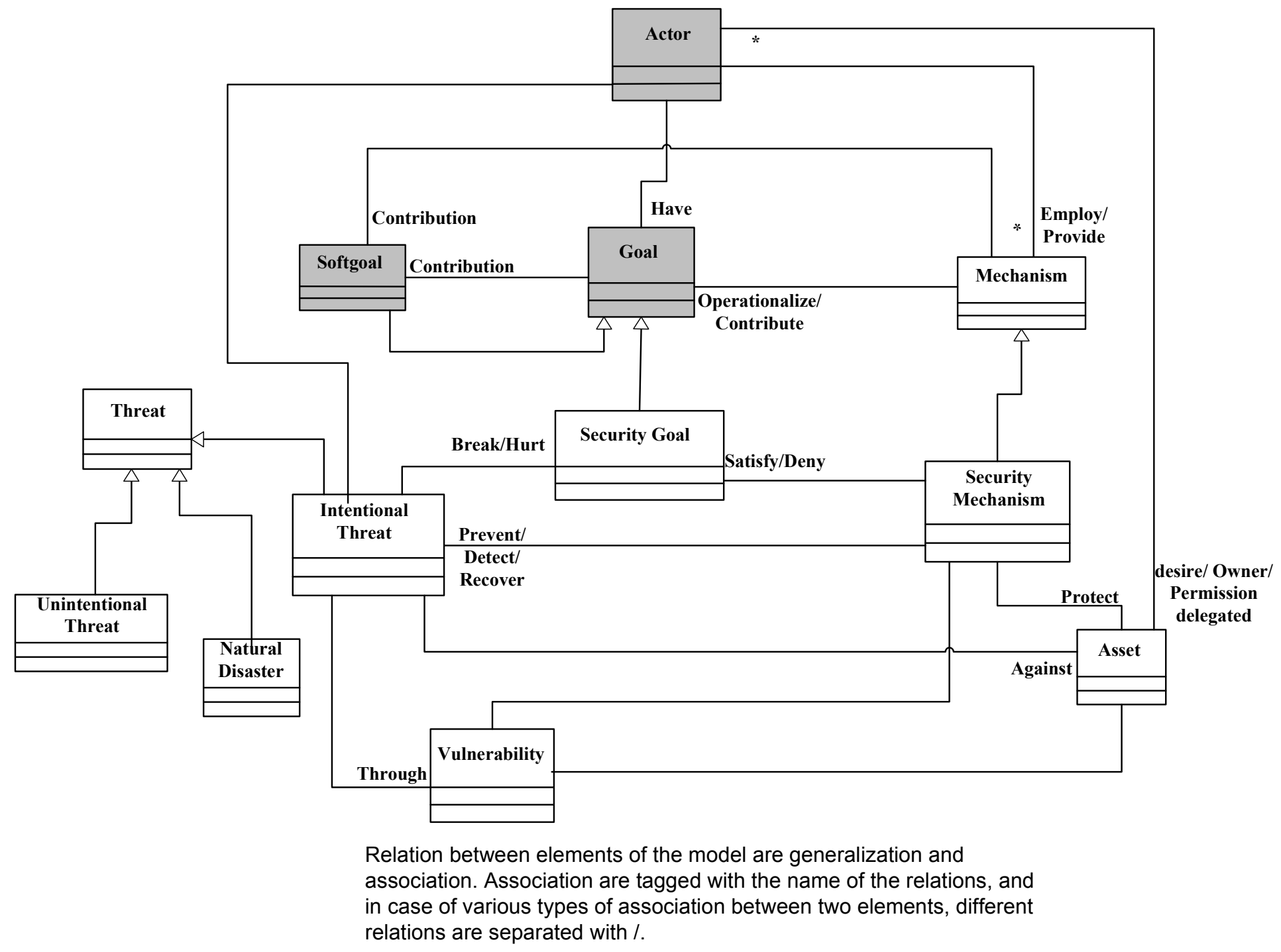

Figure 3-1. Meta-model of security concepts used in proposed modeling notation. The highlighted elements are taken from $i^{*}$ meta-model directly.

\subsection{The i* Framework}

In recent years, agent and goal oriented approaches in Requirements Engineering have emerged as new approaches to analyzing and designing of complex software systems. Examples of agent and goal oriented approaches and frameworks are the $i^{*}$ 
framework [19], and Tropos [20], and goal oriented ones are KAOS [17], the NFR framework [18]. In $\mathrm{i}^{*}$ and Tropos approaches, systems are modeled as intentional agents collaborating or competing with each other to achieve their goals. Security issues arise when some actors, while striving to achieve their own goals, intentionally or unintentionally threaten other actors' goals. Therefore, agent and goal oriented approaches provide a suitable basis for dealing with competing goals of multiple actors. Figure 3-2 summarizes the modeling constructs of the $i^{*}$ notation.

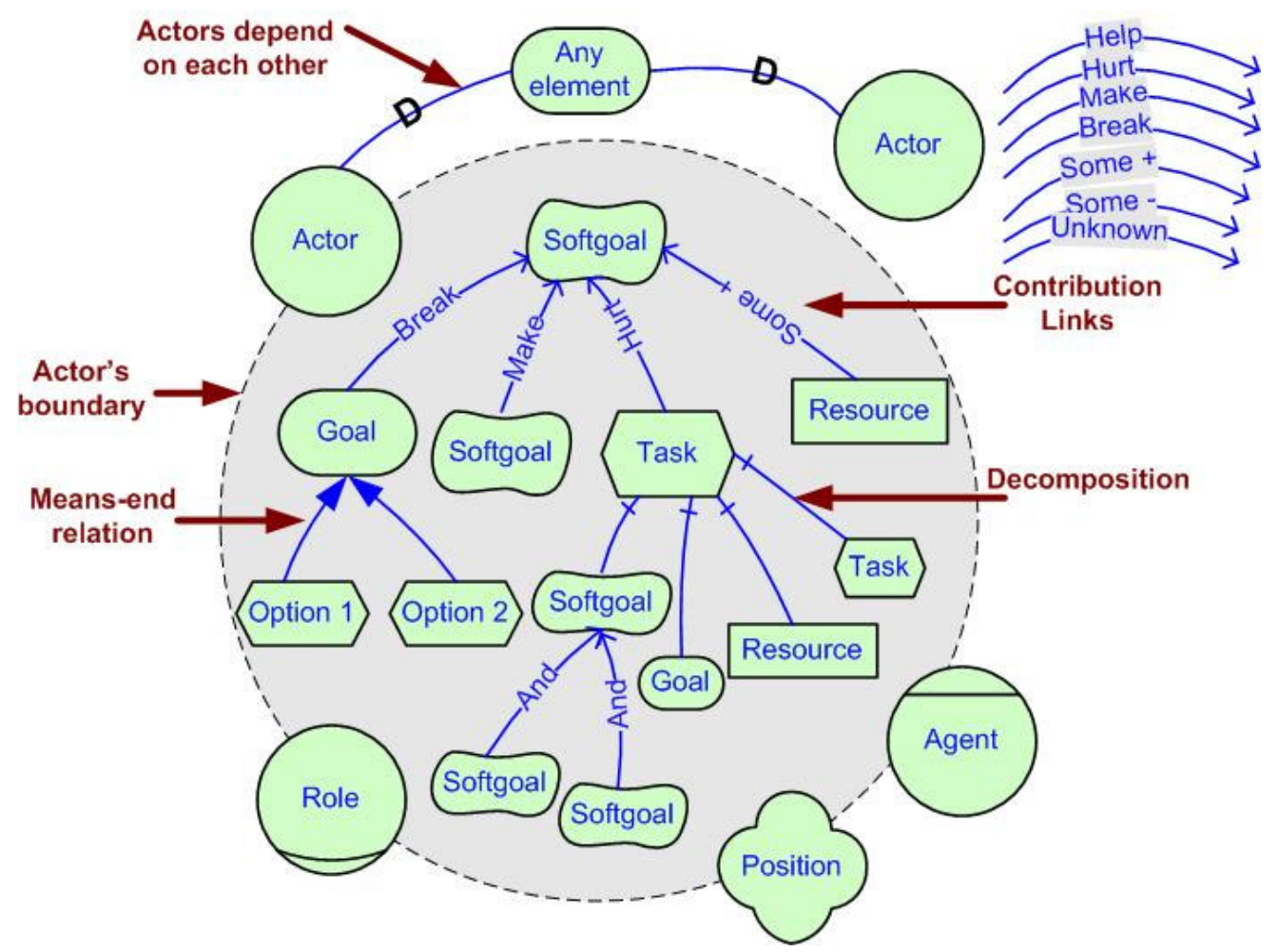

Figure 3-2. Summary of $i *$ modeling elements

The ability of $\mathrm{i}^{*}$ to model agents makes it ideal for understanding of trust, privacy and security, as this type of analysis involves the conflicting intentions of different social entities [34]. Such issues are addressed using $i^{*}$ in $[21,22,23,24,25,26]$. In addition, the $\mathrm{i}^{*}$ provides means to express the trade-offs using the contribution links. The $\mathrm{i}^{*}$ 
notation has the main modeling constructs to express the concepts in the proposed metamodel. However, the $i^{*}$ notation lacks explicit modeling constructs for concepts such as threats and vulnerabilities. In this section, we propose some extensions to the $\mathrm{i}^{*}$ notation which provide conceptual structure for modeling and analyzing security trade-offs.

\subsection{The Security Trade-offs Modeling Notation}

In this section, we propose some extensions to the $i^{*}$ notation. The extended notation provides conceptual structure for modeling and analyzing security trade-offs.

\subsubsection{Malicious Actor, Goals and Tasks}

Actors depend on or compete with each other to achieve their goals. Meanwhile, malicious actors try to achieve their own goals. Representing a malicious actor with a different modeling construct in $i^{*}$ was first employed in [21], by highlighting them from other actors with a black shadow rectangle behind the malicious actors' graphical element. This notation was used to model malicious goals in [22] as well. We make use of this notation in which malicious goals, softgoals, tasks, and actors are highlighted by a black shadow rectangle. By distinguishing malicious modeling elements from nonmalicious ones, we emphasize studying the attackers' goals and tasks. Although attacker's behavior might be partially unknown and generic, an important aspect of tradeoff analysis depends on studying attackers' options and the risks they pose to other actors' goals.

A security threat is any malicious behavior that interferes with the achievement of other actors' goals. For example, in Figure 3-3, Malicious Employee is the malicious actor whose goal is to Commit a fraud, either through the local network or over the Internet. Threats 
might be unintentional or caused by natural disasters. In this paper, we mainly focus on the security threats caused by actors with malicious intent.

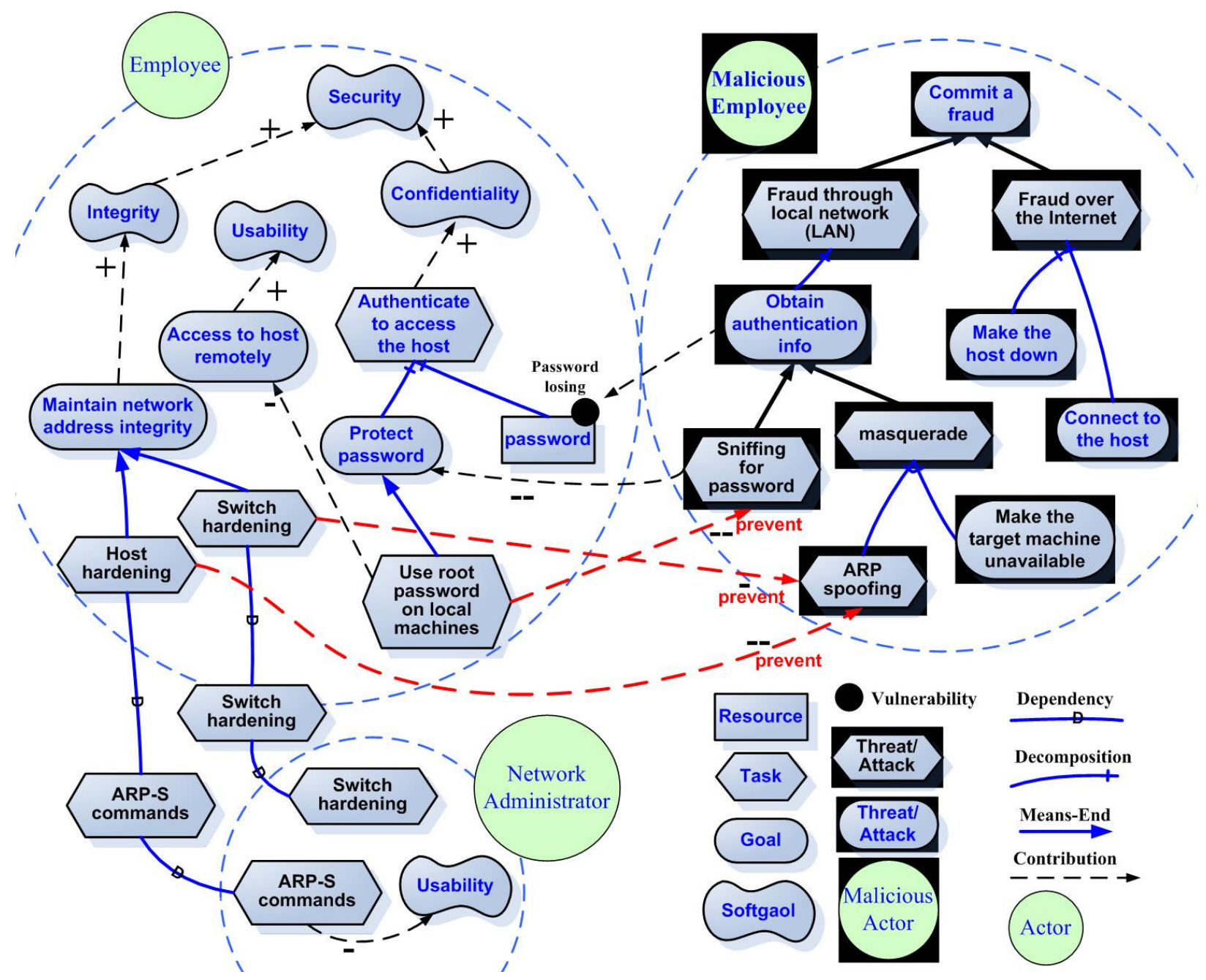

Figure 3-3. Example of a multi-actor system modeled using the proposed notation. Red lines (in electronic copy) do not bear different semantic, and are used to facilitate browsing the model

\subsubsection{Assets, Services and Vulnerabilities}

An asset is any thing that has a value for the organization [33]. Physical resources, information, and people can be counted as assets. In this way, the asset concept is well matched with the "resource" modeling element in $i$ *. Assets can be the services an 
organization offer or receive, and in this case, can be represented by tasks or goals that actors offer to the "depender" actors.

In security analysis, a vulnerability point is any weakness in, or back door to the system [33]. For example, it is said that buffer overflow and password cracking are the most common vulnerability points of many computer systems [32]. Generally, a vulnerability point corresponds to an asset or service, and attackers usually try to achieve malicious goals through a vulnerability to reach an asset. In the $i^{*}$ notation, tasks are usually decomposed to goals, softgoals, other tasks, and resources. In this way, harm of an attack can be indicated by the cost of the failed task that relies on the compromised assets. In a similar approach in $[12,11]$, threats are described in terms of assets, the action that exploits the assets, and the subsequent harm.

Although vulnerability that arises from dependencies among actors is a fundamental concept in $i^{*}$ in [22], there is no explicit modeling construct in $i^{*}$ to represent vulnerability points. We add the vulnerability point modeling element to $i^{*}$, accompanied with a graphical notation to connect a vulnerability point to the corresponding attacks, and to attach it to a resource. For example, in Figure 3-3, to protect confidentially employees are authenticated by the host. Hence, Password is one of the employees' assets they need to protect. On the other hand, Password losing is one of the most important vulnerability points in computer systems. Sniffing for password is an attack against the goal of Protect password. Through this attack and Password losing vulnerability point, the goal of Fraud under someone else's name can be satisfied, and the attacker gains a valuable asset: the Password. 


\subsubsection{Security Mechanisms}

In a number of security requirements modeling frameworks such as $[25,26]$, security mechanisms are distinguished from other tasks and goals. In this work, security goals are not modeled in a different way from other goals, because the trade-off analysis method does not treat security goals differently. The only difference of security mechanisms is the type of relation they have with attacks which is discussed in the following sub-section.

\subsubsection{Relation between Attacks and Security Mechanisms}

In the $\mathrm{i}^{*}$ notation, relation between softgoals and other elements is modeled by contribution links [19]. If an element hurts a softgoal, yet is not enough to prevent it, the contribution link type is -. If the element is sufficient to prevent a softgoal, the contribution link type is --. This qualitative approach is used to model the impact of attacks on softgoals and the impact of security mechanisms on malicious tasks and goals. In security engineering, various mechanisms have different effects on attacks. Contribution of mechanisms to attacks are categorized as 1) Prevent 2) Detect 3) Recover [33].

These categories are added as attributes on the contribution links. "Detect" and "Recover" contribution links may partially mitigate the effect of attacks. Mechanisms which are related to the attacks with "Detect" contribution links cannot control any attack. Similarly, "Recover" contribution links indicate that the mechanisms can not control the attack either, but the mechanism would be used to recover the system after the attack. This link would be useful to express availability and integrity goals that rely on 
recovering the system after the failure. To sufficiently counteract an attack, security mechanisms must be related to the attack with a "Prevent" contribution link.

\subsubsection{Expressing Trade-offs by the Proposed Conceptual Structure}

The proposed approach provides the means to model goals, and trace them back to the source actors. In this approach, trade-offs among goals are modeled by contribution links. Through contribution link types of,,---+ and $++[18]$, the qualitative effect of alternative solutions are propagated to the other goals. The $i^{*}$ notation offers the conceptual structure to model trade-offs between refined sub-goals of high level goals as well. For example, in Figure 3-3, the employee can Use root password on local machines to completely prevent the attack of Sniffing for password [32]. However, this security solution contributes negatively to the Access to host remotely goal, and it has negative influence on the Usability softgoal consequently. In this way, the trade-off among usability and security is modeled through relationships among their refined sub-goals.

\subsection{Trade-off Analysis and Decision Making}

In the previous section, we proposed a conceptual modeling technique for modeling security trade-offs. In this part, we propose a trade-off analysis method for use with the trade-off model. Designers need to balance the trade-offs to mitigate the security risks and yet satisfy the goals of multiple actors. A goal is at risk when it may be denied (partially or fully) by the successful behavior of malicious actors. Partial or full denial of goals is expressed through contribution links of type - and --. Hence, for trade-off analysis, designers need to examine available alternative security solutions, and verify the 
impacts of each one on attacks and goals to finally select the one which fits with the goals of multiple actors.

Goal model evaluation is the procedure to ensure that actors' top level goals are satisfied by the choices they have made [34]. Goal model evaluation is a means to decide among existing alternative solutions by assessing the goal achievement for each alternative. The security goal model evaluation, consisting of interactive qualitative reasoning, is based on the method proposed in [18] and refined in [34]. The evaluation examines if goals and softgoals are partially or fully satisfied (denied). Figure 3-4 depicts the proposed security trade-off analysis procedure.

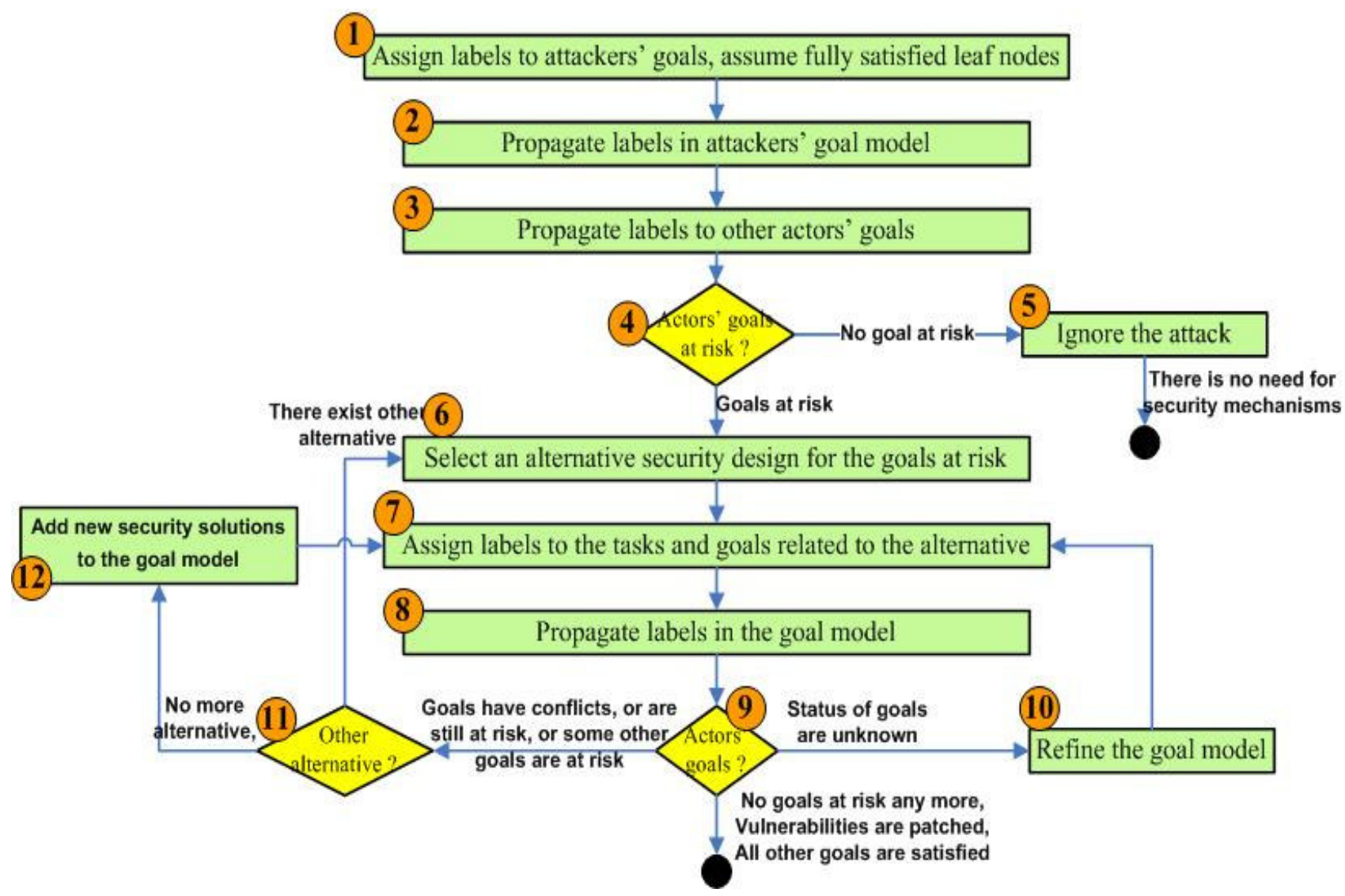

Figure 3-4. Security trade-off analysis procedure 
In the first step, the evaluator assumes that attackers are successful in performing tasks and satisfying their goals, since attackers are usually external actors that designer has no sure knowledge of their abilities and skills. Therefore, the leaf nodes in attackers' goal model are labeled fully satisfied. This assumption does not imply that the risk of attacks is definite, as it is possible that evaluation of attackers' goal model yields to denial of higher goals of attacker. The leaf labels are propagated to upper goals. Once the impact of malicious actors' behavior is propagated to the entire goal model, the evaluator assigns labels to the tasks and goals that operationalize security mechanism (step 7). This label indicates the evaluator's judgment about the success of the actor in performing a security task or achieving a security goal. This judgment could be based on knowledge of previous experiences, empirical studies, or subjective knowledge [6].

In step 9, the goal model indicates which goals are fully or partially satisfied or denied for the examined security solution. The procedure iterates until a security design solution is found that, based of the evaluator's perception, satisfies an acceptable configuration of goals. However, the evaluator may prefer to examine further alternatives to select the security design solution that satisfies more goals. After evaluating an alternative, the status of some goals may be unknown, prompting the designer to elaborate on the models (step 10). In case of conflict among goals, other alternatives should be examined to resolve the conflicts (step 11).

Propagation of the labels is based on the contribution types and rules summarized in Table 2. [34] provides details about aggregation rules for multiple contributions. The rules provided in Table 3-1 are valid only for the "Prevent" contribution type, as we 
discussed earlier that recovering from, or detecting an attack do not lead to controlling the attack.

Table 3-1. Evaluation labels and propagation rules from [18, 34]

\begin{tabular}{|c|c|c|c|c|c|c|}
\hline \multicolumn{2}{|c|}{ Child Node } & \multicolumn{5}{|c|}{ Contribution Type (Prevent) } \\
\hline Label Name & Symbol & ++ & + & - & -- & $?$ \\
\hline Satisfied & $\sqrt{ }$ & $\checkmark$ & $\sqrt{ } \cdot$ & $\underline{x}$ & $x$ & $?$ \\
\hline Weakly Satisfied & $\sqrt{ }$ & $\sqrt{ } \cdot$ & $\sqrt{ }$ & $x$ & $x$ & $\dot{?}$ \\
\hline Conflict & 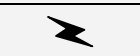 & $>$ & 2 & 2 & 2 & ? \\
\hline Unknown & ? & ? & ? & $?$ & $?$ & ? \\
\hline Weakly Denied & $x$ & $x$ & $x$ & $\sqrt{ }$ & $\sqrt{ }$ & ? \\
\hline Denied & $x$ & $x$ & $x$ & $\sqrt{ }$ & $\sqrt{ }$ & $?$ \\
\hline
\end{tabular}




\section{Case Studies}

In developing the proposed extended $i^{*}$ notation, we worked out a number of examples using NIST guidelines [35] and security engineering knowledge in [32]. In addition to those example cases, we applied the notation to three example cases originally used by other authors to illustrate their techniques for doing security trade-off modeling and analysis. In the first example case, we modeled and analyzed the GA system, a patient and physician supporting system, which was modeled and analyzed in [22]. The second case study is eSAP system, an agent-based health and social care system, which was used as the case study system in $[24,25,26]$. In the third example case system, we modeled and analyzed a simple Course Registration system, using the proposed extensions to the $i^{*}$ and the framework proposed in [27].

The main objective of the case studies is to evaluate and compare existing approaches with the proposed method in terms the expressiveness of trade-offs models and comprehensiveness of the trade-off analysis results. The detailed case studies and comparison of resulting models would reveal the strong and weak points of the modeling techniques and analysis methods. Each case study is organized into three subparts: nonmalicious goal model, malicious goal model, and security goal model accompanied with trade-off analysis results. Each part presents the original models of case study system from the original source, models of the case system developed using our proposed method, and a comparison between the two modeling and analysis approaches. The third case study which compares the ATAM approach with the proposed technique is not broken down into the above mentioned three subparts, since the framework of scenarios and tactics [27] do not provide means to model malicious goals. 


\subsection{Case Study One: Guardian Angel Project}

GA is a patient and physician supporting system using software agents. This information system is centered on the individual patient instead of the provider, in which a set of "guardian angel" (GA) software agents integrates all health-related concerns, including medically-relevant legal and financial information, about an individual. This personal system will help track, manage, and interpret the subject's health history, and offer advice to both patient (in the case study Abby plays patient role) and provider. Patients provide the medical information to a PDA which is connected to other systems such as the GA-Home, GA-hospital and peer GA-PDA.

The methodological framework for dealing with security requirements in [22] is illustrated with the examples of designing GA software agents. The requirements elicitation process in [22] starts from identifying and modeling actors and their goals and tasks. In parallel, vulnerabilities, malicious actors and intents are identified and integrated into the goal models. Finally, countermeasures for attacks are chosen and added to the models and process ends with countermeasure analysis.

\subsubsection{Non-Malicious Goal Model}

The approach in [22] takes advantage of network of Strategic Dependency (SD) to model the dependency among actors and their goals which provides the basis to analyze the opportunities and vulnerabilities. Figure 4-1 gives a part of the dependency relationships (SD model) in [22]. In this model, the goal refinement inside the GA actors' boundaries such as GA-PDA actor is not considered, and the focus is on studying Aebby's goals which depends on GA-PDA agent. However, to analyze security trade-offs for alternative solutions, the designer needs to study the impact of each alternative security 
mechanism on goals of all actors; therefore, developing a refined goal model for each actor is essential. Figure 4-2 gives a detailed refinement of GA-PDA actor's goals.

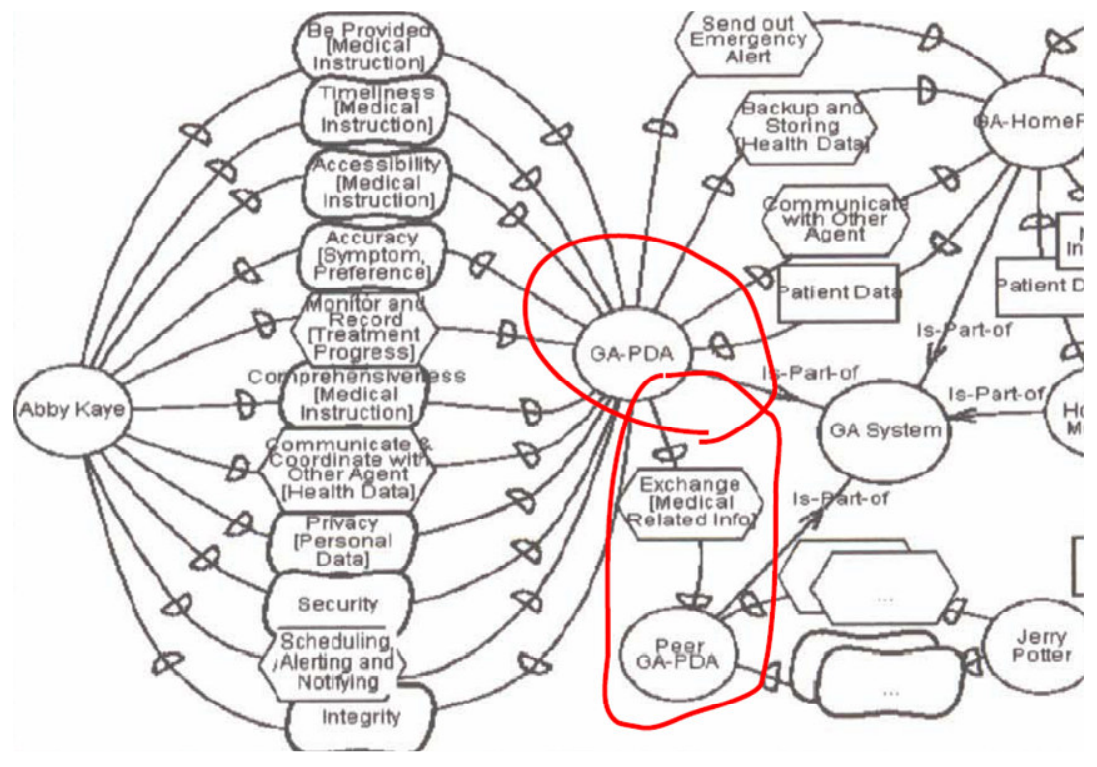

Figure 4-1. A part of dependency relationships in the GA system, original model is obtained from [22]

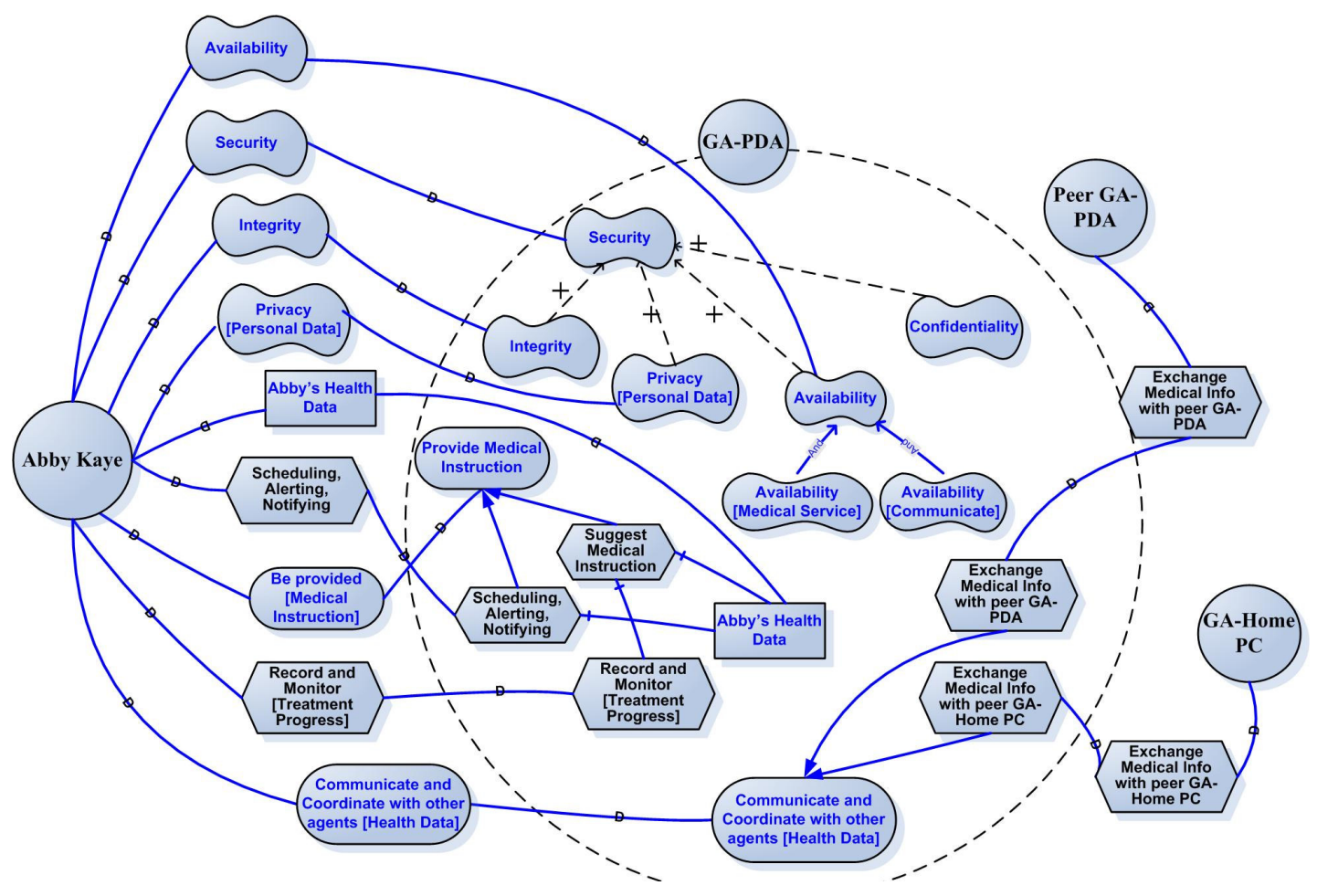

Figure 4-2. Detailed refinement of GA-PDA actor's goals. The model enables the designer to relate attackers and security goals to all refined GA-PDA goals and tasks 


\subsubsection{Malicious Goal Model}

In [22], the basic premise for attacker analysis is that all the actors are assumed guilty until proven innocent. In this way, each actor of the goal model can be a potential attacker, and each one is studied in two roles: its regular role, and its potential malicious role. In vulnerability analysis, each dependency is analyzed as a potential threat against the system. One of the actors in the dependency relation is substituted by its corresponding attacker, and vulnerable dependencies are highlighted with a black shadow rectangle. Figure 4-3, depicts the dependency vulnerability analysis in [22].

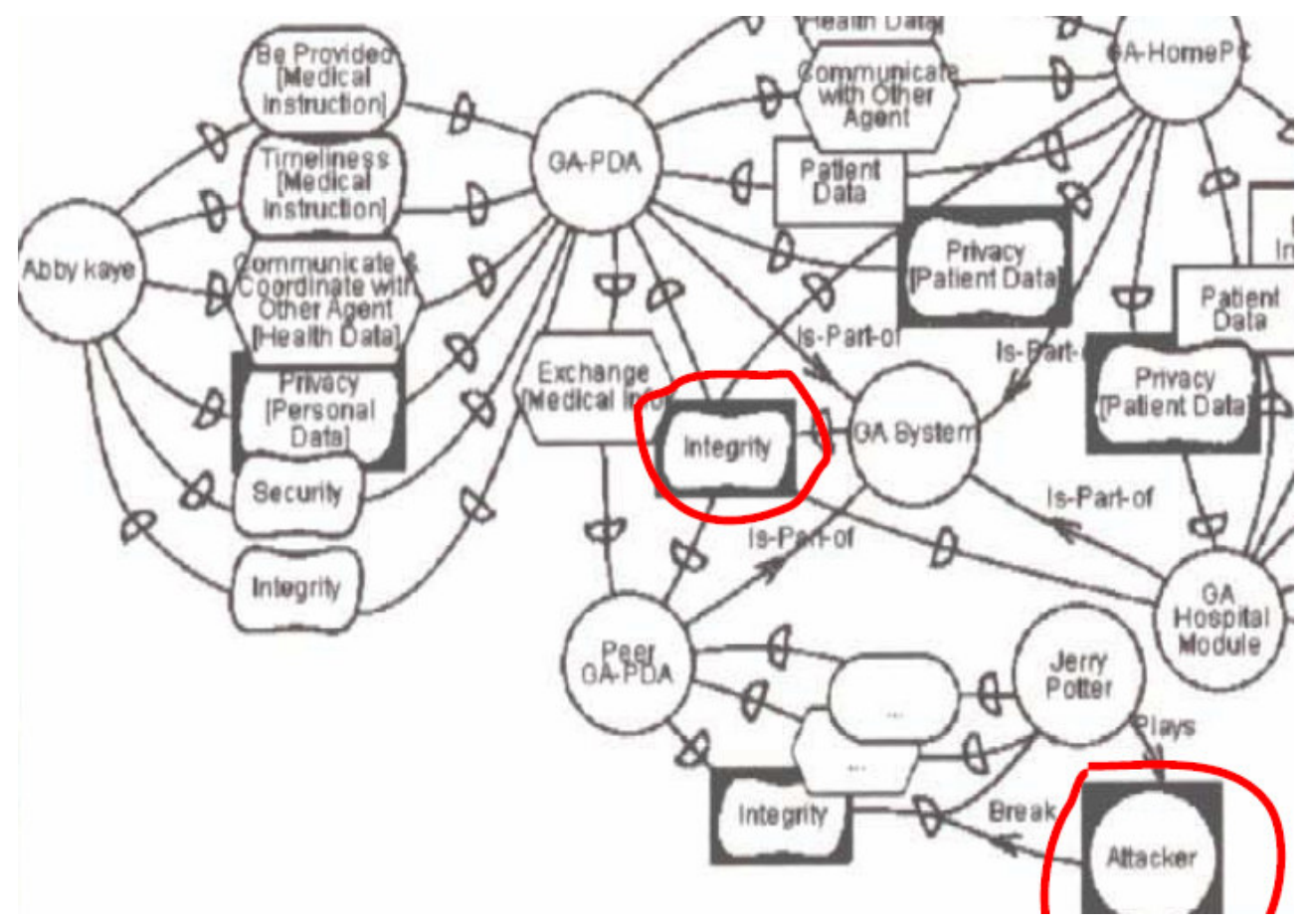

Figure 4-3. A part of dependency vulnerability analysis of the GA system, original model from [5]. Each dependency is examined as a potential threat against the system. Each actor plays role of a potential attacker as well as its regular role 
For each vulnerable dependency, alternative attacks are identified and modeled as tasks that contribute to the malicious goal. Figure 4-4 illustrates the original attack model of GA system in [22].

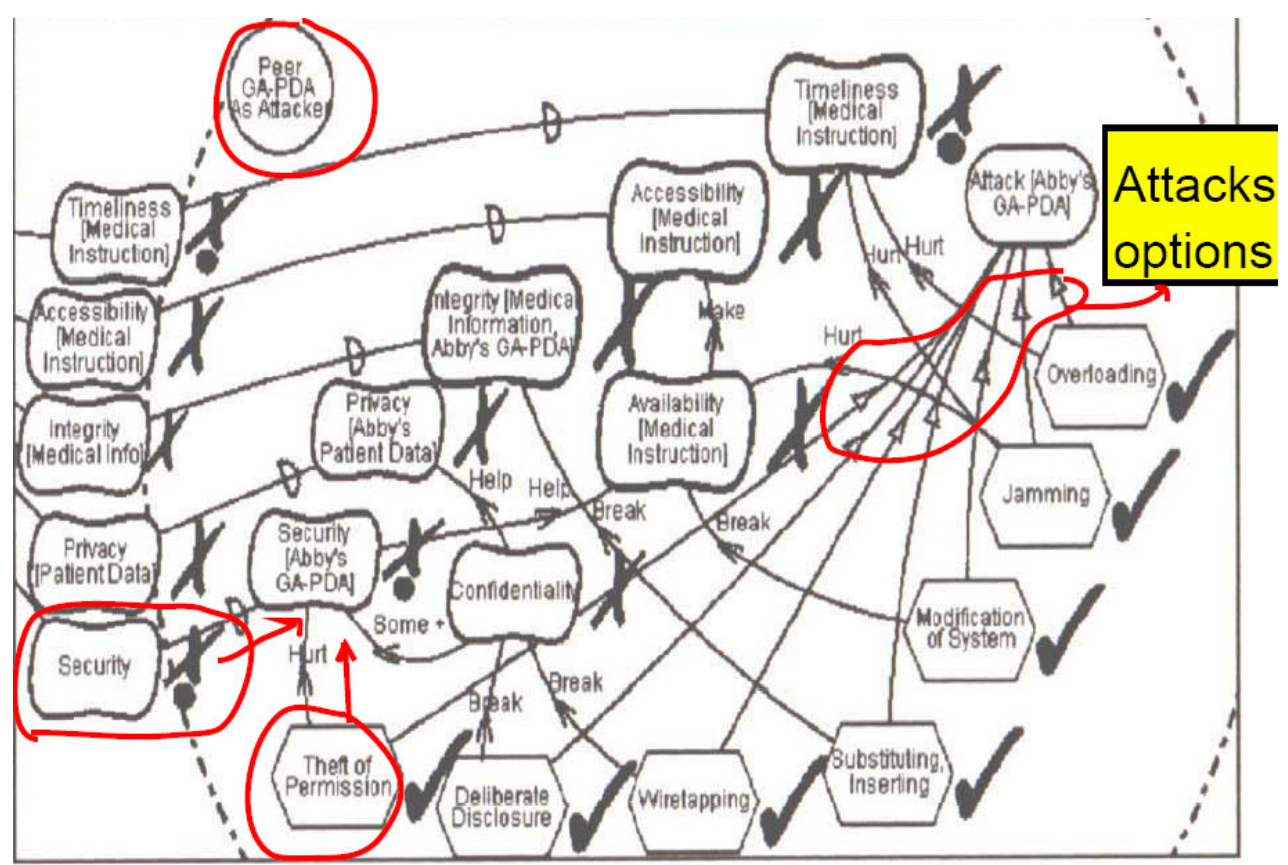

Figure 4-4. Attack and threats identification, original model from [22]. Malicious tasks and affected goals are located inside the boundary of the malicious actor. Malicious goals and tasks are not distinguished from regular goals

We now consider the weaknesses in the approach taken in [22] and compare it with the proposed approach. Analyzing the dependencies as the potential vulnerability points in [22] is useful, but it is not enough to uncover the potential threats imposed by external entities. The resulting models do not capture goals and intentions of the attacker. However, the designer needs to know how the attacker may use the vulnerabilities as a backdoor to the system, since deciding among different countermeasures depends on the attacker's goals. For example, the designer needs to differentiate between goals of a professional hacker and intentions of a curious kid to 
select proper security mechanisms. The approach in [22] neglects analyzing the alternative attacks that each one requires specific security safeguard. A major problem with the modeling approach in Figure 4-4 is that both security safeguards and attacks are located inside the boundary of the attacker. Besides, the contributions of security mechanisms on goals of other actors are not modeled, and it is not specified which actor(s) performs the security tasks.

Figure 4-5 presents the attack model using the proposed extensions to $i^{*}$. In this view, potential attacks, malicious goals, and sub-goals are taken into account. The impact of each malicious goal and task is modeled on all goals of the attacker. The resulting goal model captures the effects of each alternative attack on attacker's goals and softgoals.

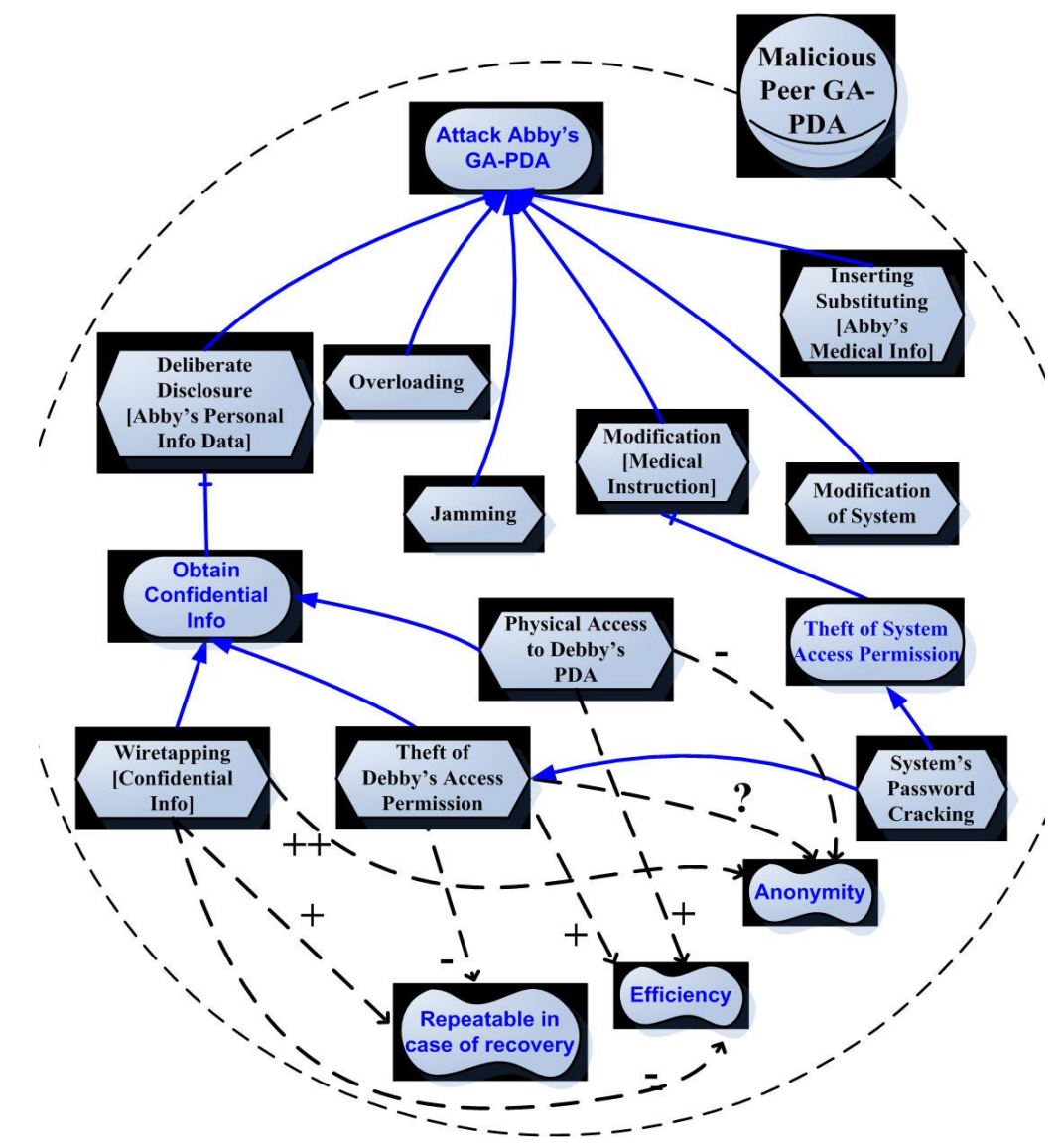

Figure 4-5. Malicious actor behavior, modeled using the proposed notation. Malicious actor goals are refined 


\subsubsection{Security Goal Model and Trade-off Analysis}

In [22], countermeasures are chosen for each attack. Countermeasures are added to the attacks model, and are related to attacks with a "break" or "hurt" contribution link. Figure 4-6 gives the countermeasure analysis model of GA in [22]. The goal model evaluation in original GA system models is limited to the evaluating threats and their corresponding safeguards. The safeguards affect other goals such as performance and usability, but generally, the resulting models in [22] do not capture these trade-offs that security mechanisms impose on other goals.

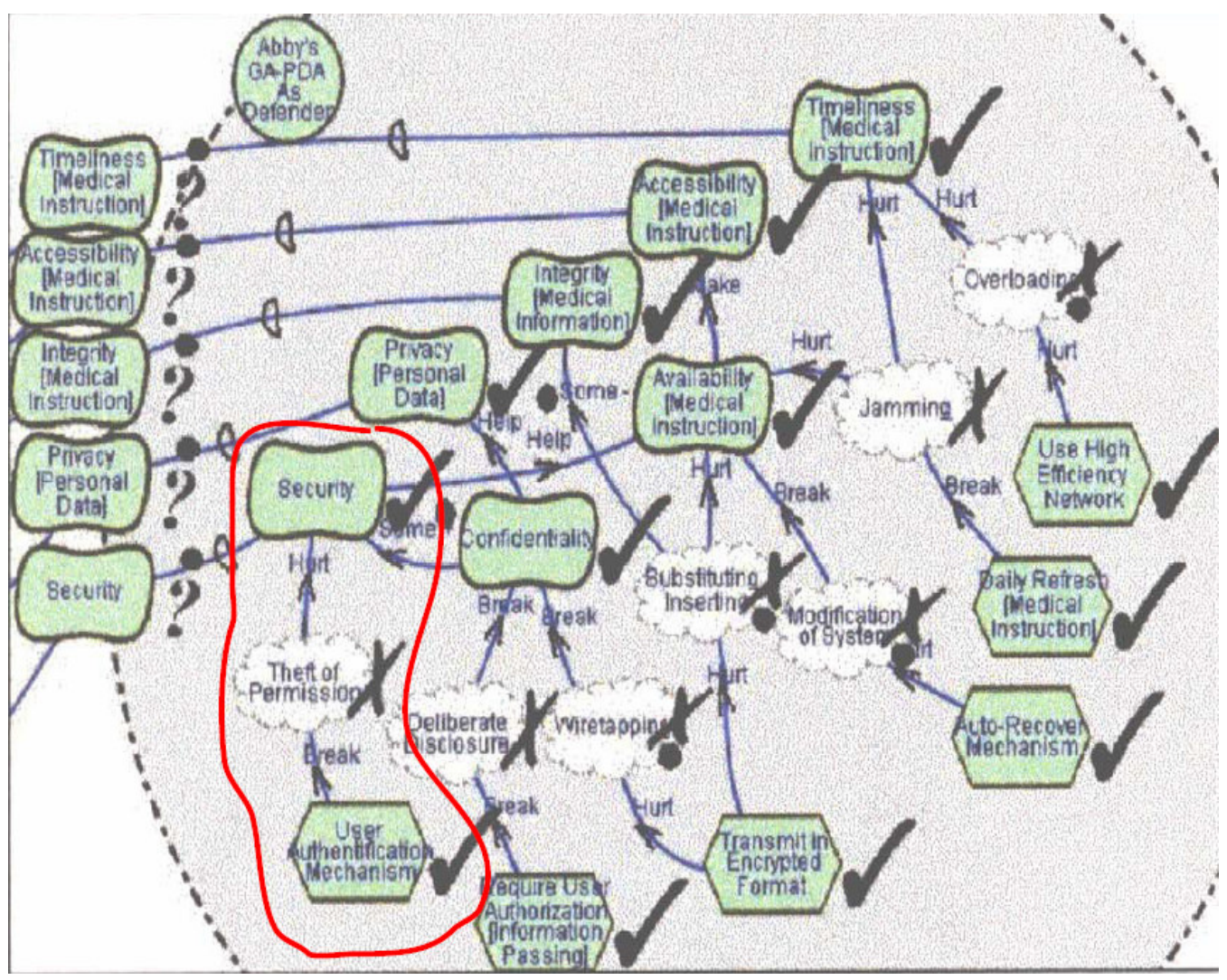

Figure 4-6. Countermeasure analysis, original model from [22]. The hypothetical threats are presented as beliefs, since their existence is based on the designer's assumption. Countermeasures contributions to the attacks are modeled, but it is not specified which actor performs the security mechanisms and what are the consequences 
In the proposed extensions to $i^{*}$, countermeasures are adopted by the actors that are at risk or by the actors which are responsible to provide security for the actor at risk. The impact of each alternative on the goals of malicious and non-malicious actors is modeled. In both approaches, alternative countermeasures are compared through goal model evaluation.

Figure 4-7 depicts resulting security trade-off model using the proposed extensions. The same security mechanisms of the goal model of Figure 4-6 are used to structure the GA-PDA goals. In Figure 4-7, trade-off of alternative mechanisms and potential attacks are modeled explicitly. It provides the designer with one picture of potential malicious behavior, alternative mechanisms, their trade-offs and consequences.

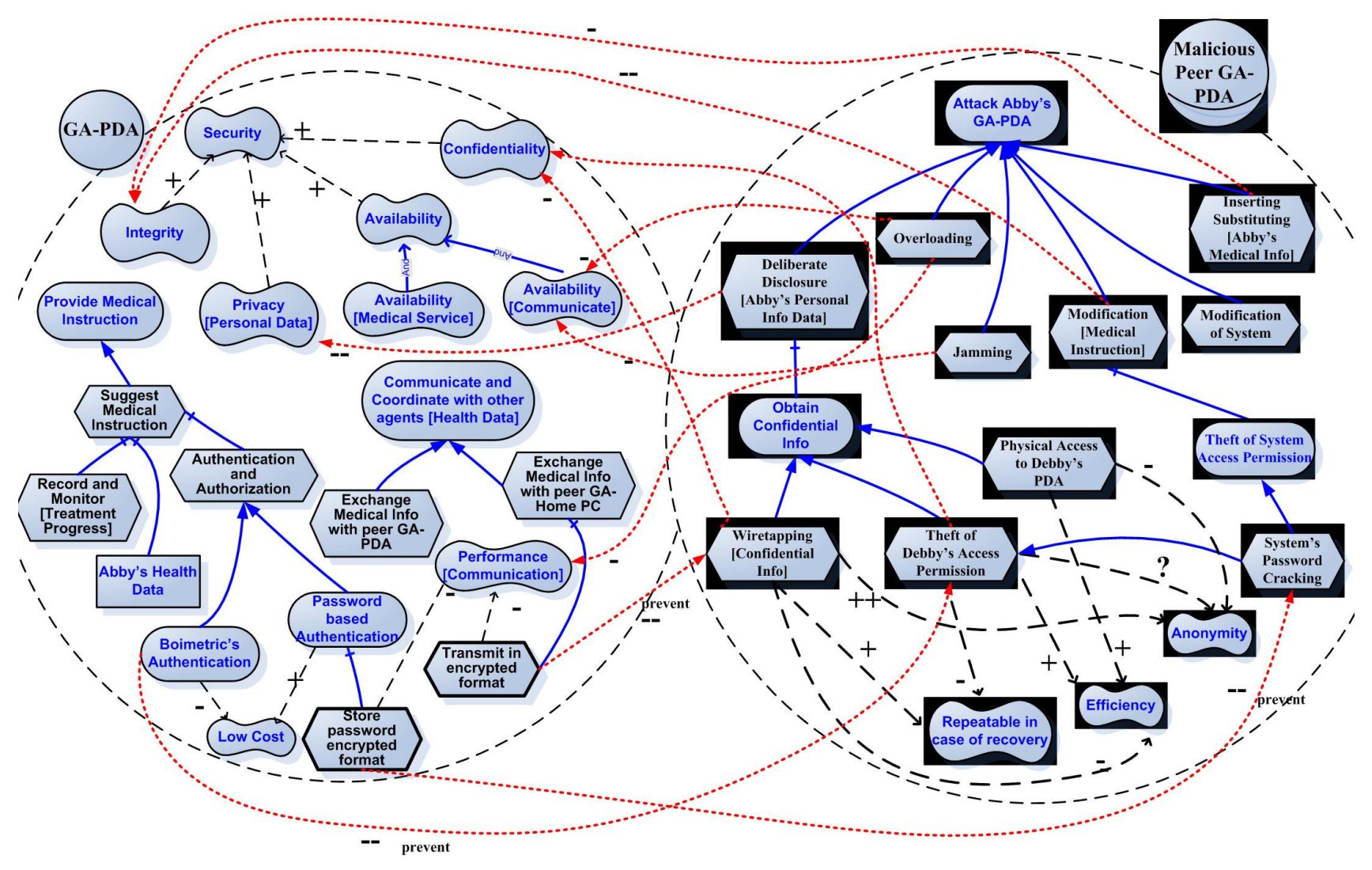

Figure 4-7. Security trade-off model, using the proposed extensions. Contribution of malicious goals and security goals are specified 
Figure 4-8 and Figure 4-9 give the security goal model evaluation results using the proposed extensions to the $\mathrm{i}^{*}$ notation. The resulting goal model captures the effects of each alternative attack on malicious and non-malicious actors' goals and softgoals. As a result, designer can evaluate risk of threats, and select a more appropriate solution for attackers' behavior based on the consequence of malicious actors' behavior.

In Figure 4-8, the evaluation steps are highlighted by number tags. This procedure starts with assigning satisfaction labels to the malicious tasks and goals. For example, in the first step, the evaluator assigns a satisfied label to System's Password Cracking. In the next step this label is propagated through the goal model.

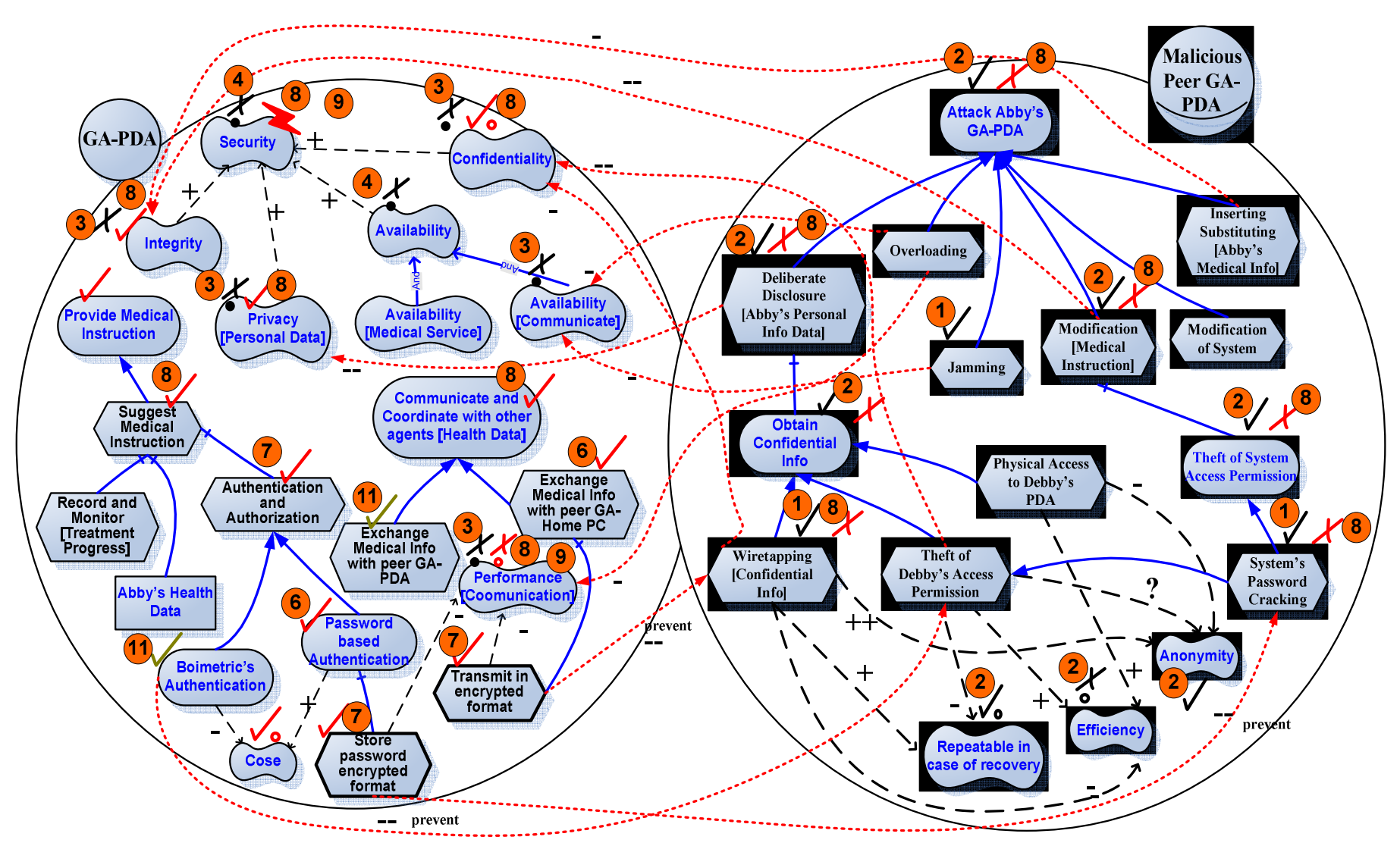

Figure 4-8. Security trade-offs analysis, using the proposed evaluation, using the proposed evaluation. The evaluation method steps are specified. 
The model in Figure 4-8 expresses that the designer decides to employ Authentication and Authorization with Password based Authentication. It yields to fully satisfy privacy and partially satisfy confidentiality, while it does not help the partially denial of performance and availability. Figure 4-9 illustrates examining the other alternative solution which is Biometric Authentication. The evaluation procedure is used to propagate values throughout the graph. Although Biometrics is a more secure authentication mechanism, and it results in fully satisfaction of confidentiality, performance is still partially denied, and in addition, low cost goal is partially denied. Hence, the designer requires examine more alternative security mechanisms to control jamming attack, which have negative impact on performance.

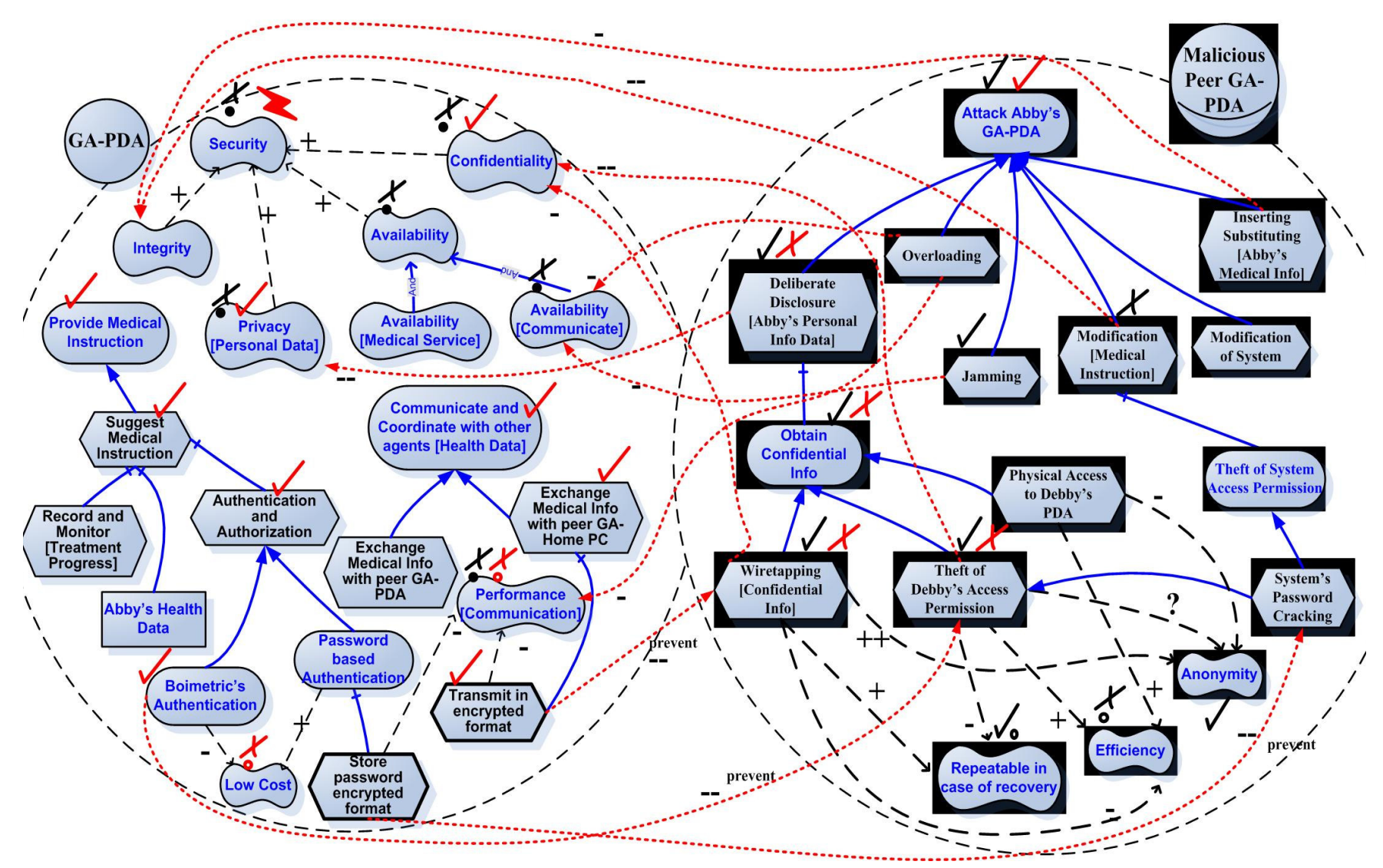

Figure 4-9. Security trade-offs analysis, using the proposed evaluation method 


\subsection{Case Study Two: eSAP System}

The second example case is the eSAP system used as the case study system in $[24,25,26]$. The electronic Single Assessment Process (eSAP) system is an agent-based health and social care system for the effective care of older people. Taking into account a substantial part of the eSAP in the original case study, the following stakeholders are defined for the case study in $[24,25,26]$ : The Older Person actor is the old person (patient) that wishes to receive appropriate health and social care. The Professional actor represents health and/or social care professionals involved in the care of the Older Person. The DoH actor represents the English Department of Health, which is responsible for the effective care of the Older Person. The Benefits Agency actor is an agency that helps the Older Person. Financially, and the R\&D Agency actor represents a research and development agency that is interested in obtaining medical information.

\subsubsection{Non-Malicious Goal Model}

The idea in $[25,26]$ is to integrate security concerns into the Tropos framework. Security constraints are modeled in a diagram called the actor diagram (Figure 4-10). Security requirements and goals are expressed by "constraints", which are represented using a cloud placed between the dependencies, at the side of the actor who has to satisfy the constraint. This approach is useful, since it enables the designer to relate the security requirements to goals and functionalities. 


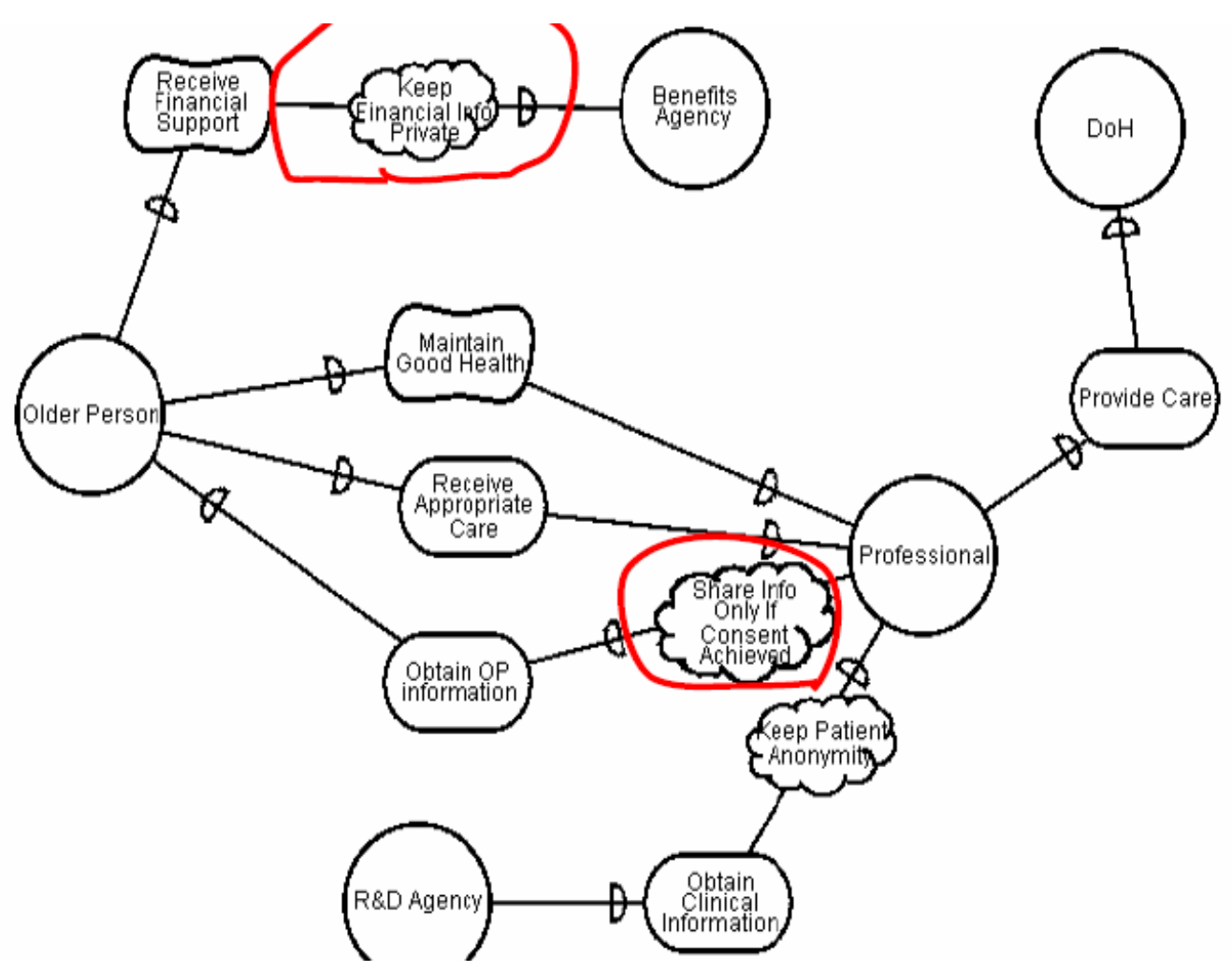

Figure 4-10. Stakeholders of the eSAP system, original model from [26]. Security constraints are placed between the dependencies to assign security requirements to the actors

Figure 4-11 gives a part of eSAP goal model which focuses on professional actor goals. It models security requirements which are expressed as constraints in Figure 4-10, in terms of softgoals. What is modeled as constraints in $[25,26]$, can be modeled as goals as well. For example, in Figure 4-10, Older Person imposes a security constraint to Professional actor to Share info Only if Consent Achieved. The model in Figure 4-11 expresses this requirement by the Privacy OP Information softgoal of Older Person who depends on Professional actor to satisfice. 


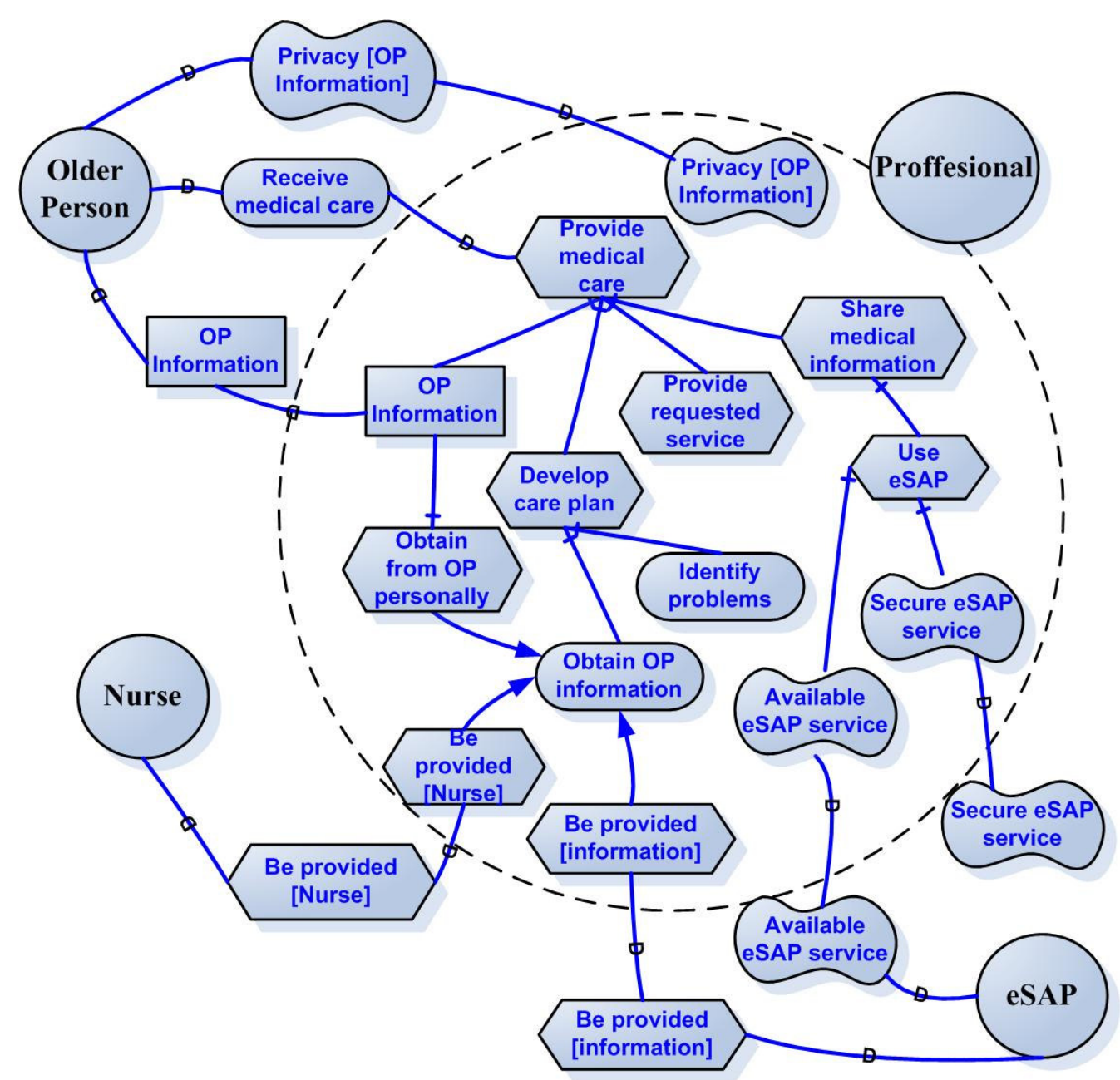

Figure 4-11. The professional actor goal model. The model includes Professional actor goals, and is similar to model in Figure 4-14

\subsubsection{Malicious Goal Model}

The approaches in $[25,26]$ introduce "security diagram" which uses $i *$ notation to model security requirements and potential threats. Figure 4-12 gives the original security diagram of eSAP, which contains the security goals, threats, and safeguards. Threats are modeled explicitly as a distinct construct in the "security diagram" using a pentagon. The impact of threats on softgoals such as privacy and availability is modeled using contribution links. 


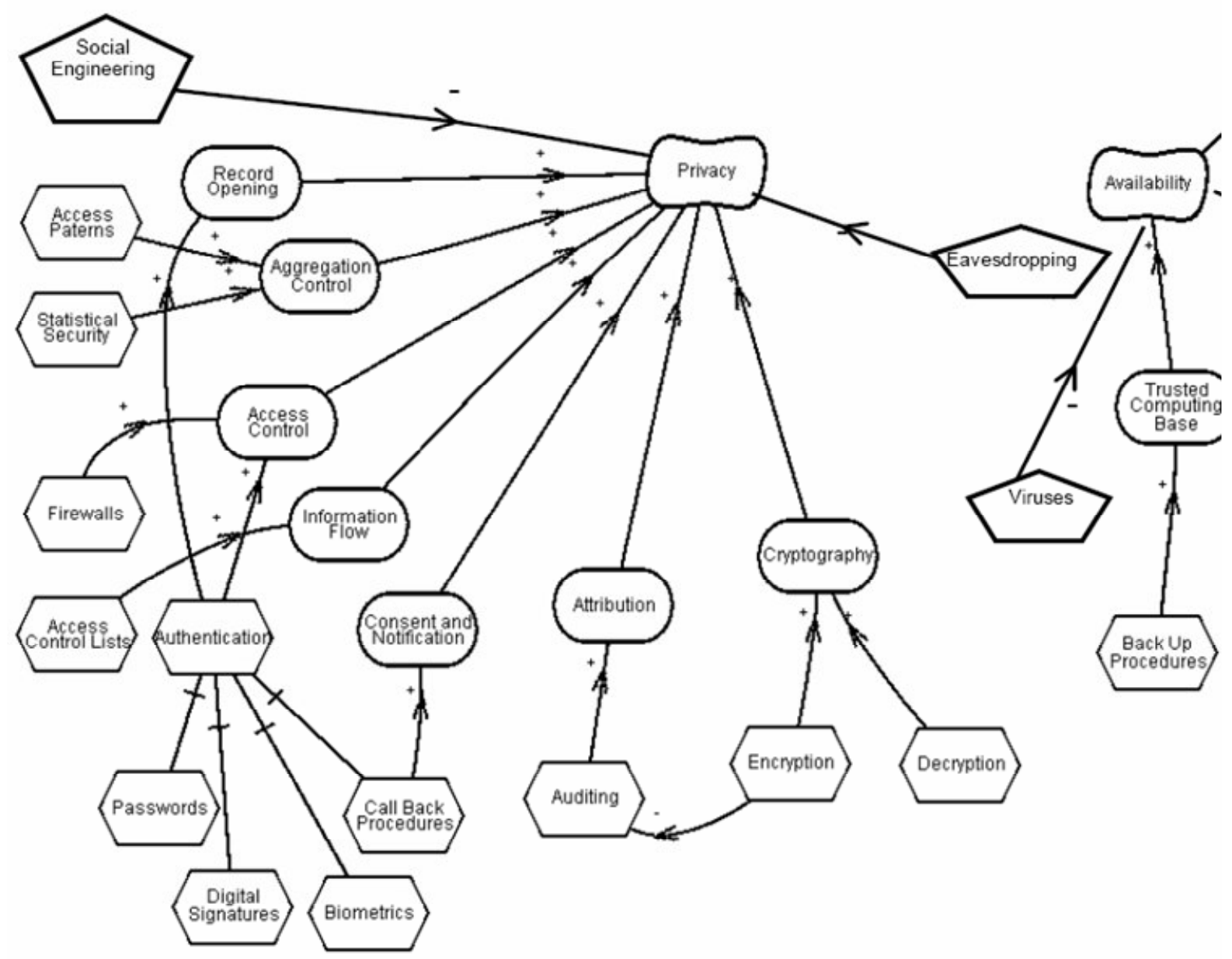

Figure 4-12. A part of the "security diagram" for the eSAP system, original model from [26]. The model does not specify which actors have the privacy goal, how the threats work against the privacy, who threaten privacy and why

However, the model does not trace the threats to the source of threats' actors, and the relation between countermeasures and threats are not elaborated in the resulting models. It is not described in $[25,26]$ how security constraints are related to the security goals and mechanisms in the "security diagram" (Figure 4-12). The main problem of the "security diagram" in the original case study is that the resulting models do not include actors; therefore, it is not clear who has the goals, who threaten them and why, who 
employs the mechanisms, and what is the impact of the mechanisms on other goals of actors.

Figure 4-13 gives the goal model of threats and affected goals, using the proposed extensions to $i^{*}$. This model includes the malicious tasks and goals and the corresponding impact on goals of professional actor.

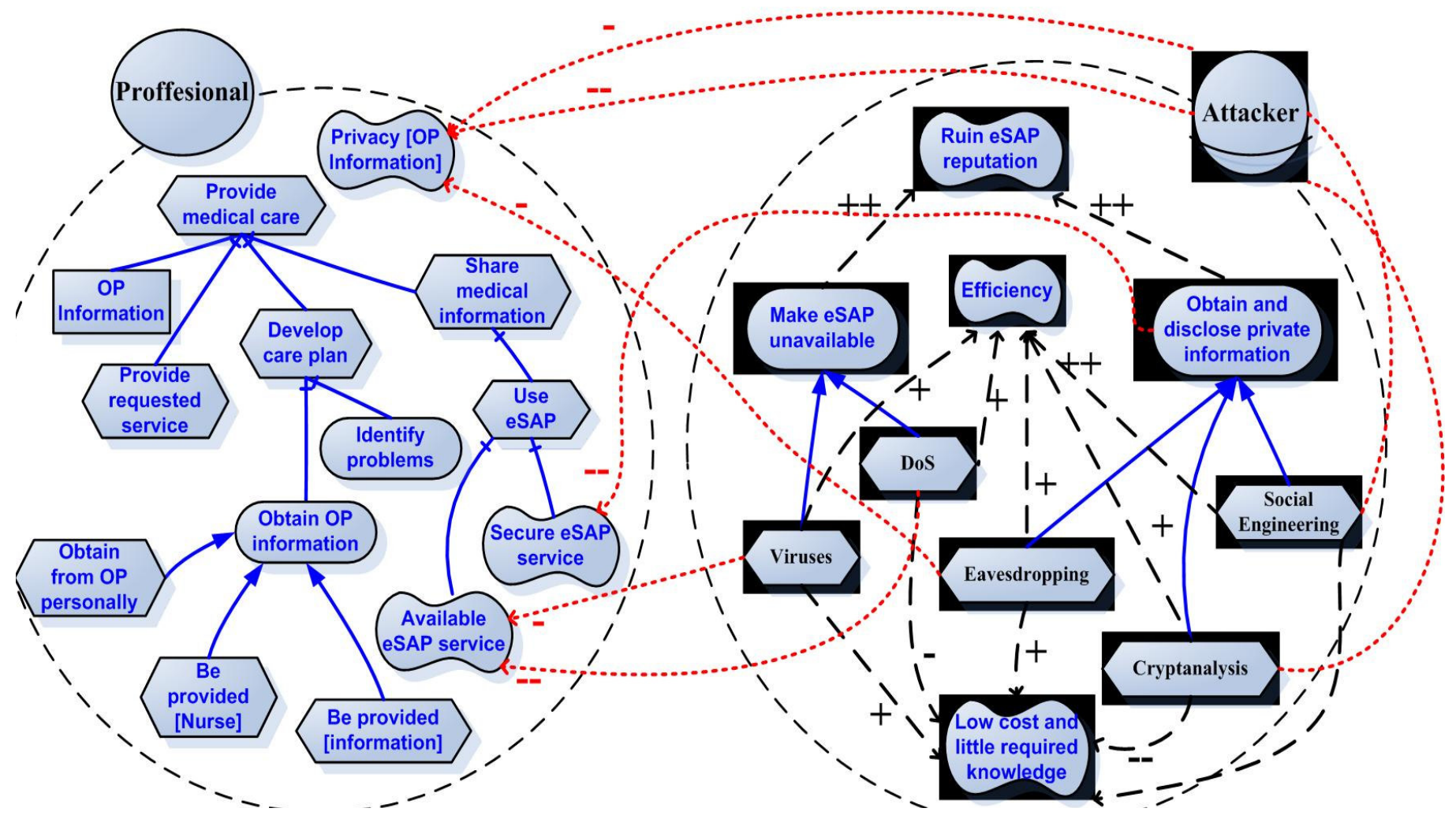

Figure 4-13. Malicious actor behavior and its relation to professional actor goals, modeled using the proposed notation

\subsubsection{Security Goal Model and Trade-off Analysis}

In $[25,26]$ approaches, once the stakeholders' goals and security constraints are identified, each actor is studied in more depth, and alternative security solutions are examined considering the constraints imposed to the actor. In [25, 26], secure entities are 
introduced to help towards the satisfaction of the imposed security constraints. However, the papers do not argue why security requirements and constraint should be expressed in a different way than other softgoals.

Figure 4-14 gives one of the actor analysis models of [26]. The actor analysis model lacks considering the impact of security goals on attacks and threats. Although the security alternatives are modeled through means-end relations, the trade-offs that they pose to other goals and softgoals are not captured in the model.

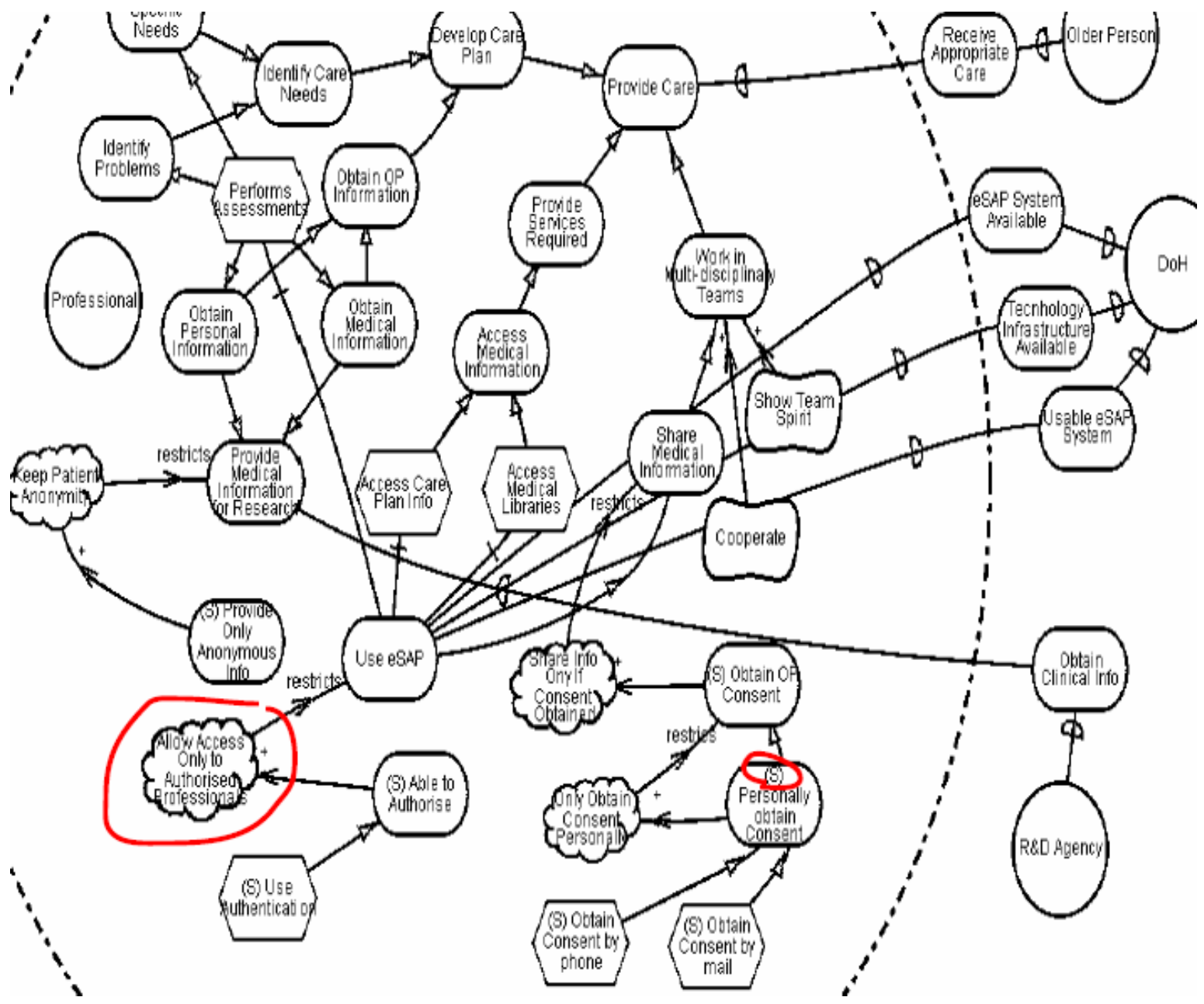

Figure 4-14. Professional actor analysis, original model from [26]. The model lacks considering impact of security alternatives on other goals and threats. The impact of threats on goals (security goals and general goals) are not expressed in the models. 
Figure 4-15 illustrates the complete goal model of professional actor, using the proposed extensions to $\mathrm{i}^{*}$. This model includes threats, security mechanisms and contribution of goals and attacks.

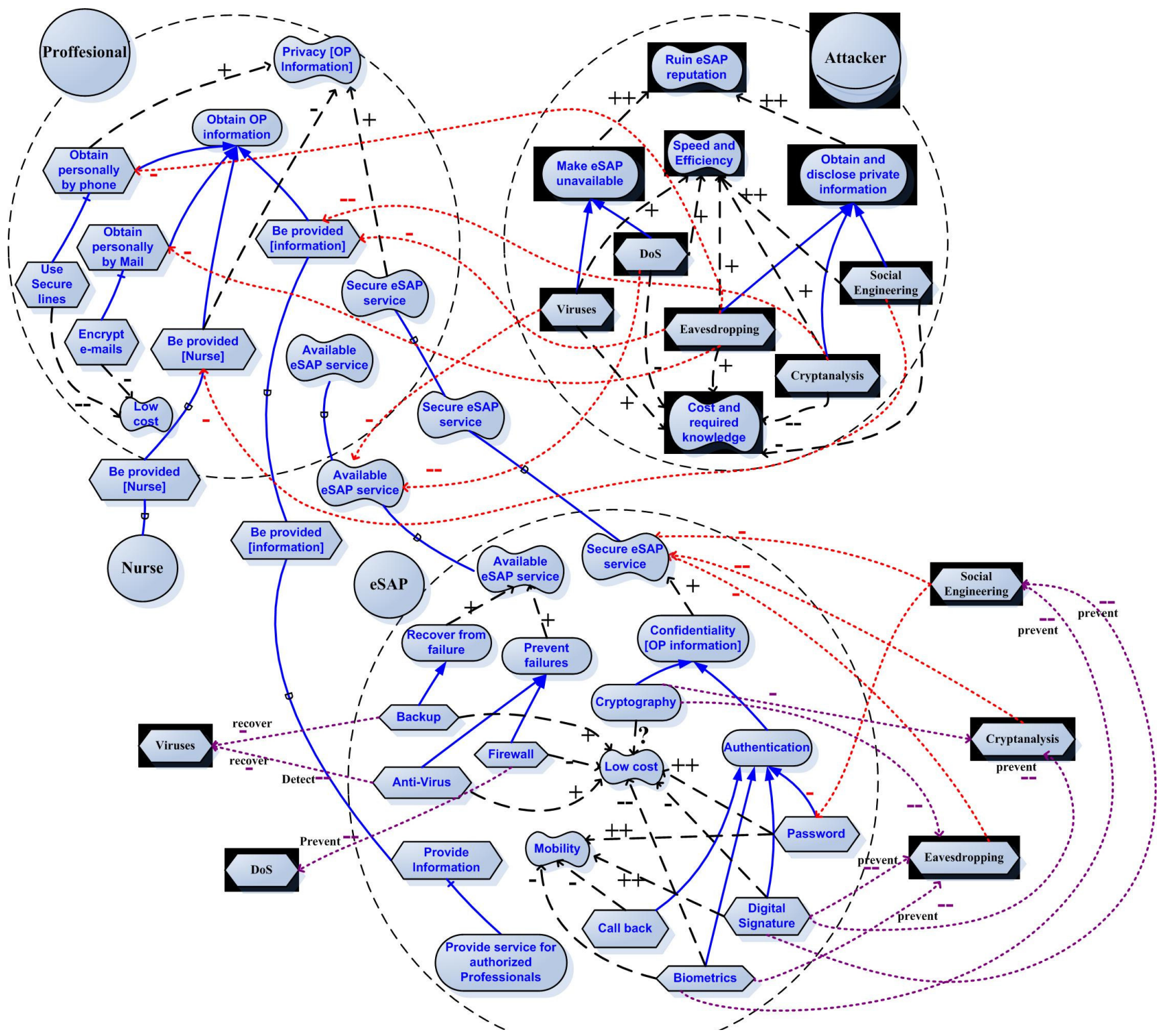

Figure 4-15. Interaction of Attacker, professional and eSAP actors. Security goals and trade-offs, modeled using the proposed notation 
The eSAP case study in $[25,26]$ doesn't provide results of goal model evaluation, since it does not capture the trade-offs in the goal models. Figure 4-16 illustrates a part of security trade-off analysis for eSAP system using the proposed approach. The steps of goal model evaluation are indicated by the tags.

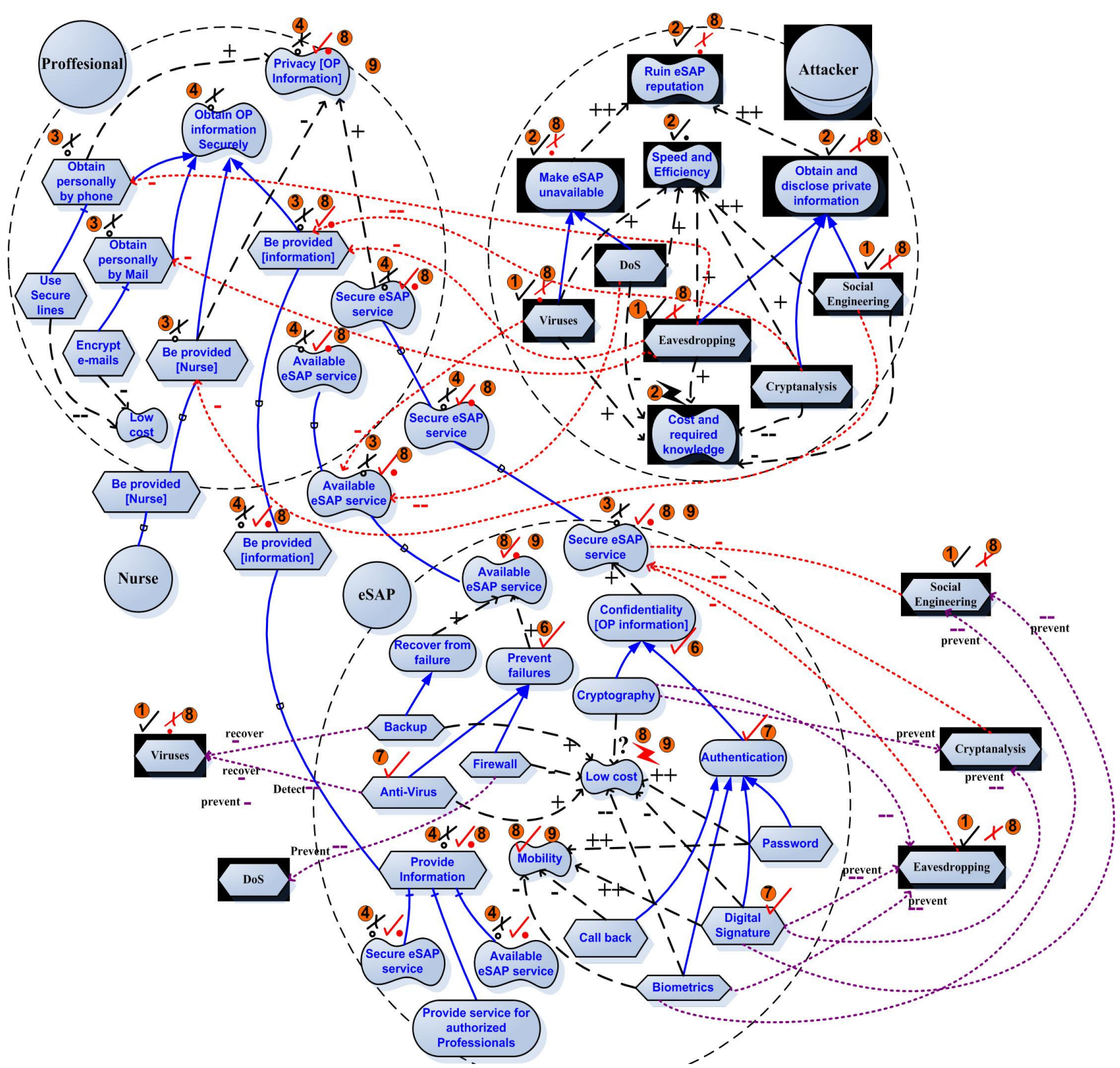

Figure 4-16. Security goal model trade-off analysis, using the proposed evaluation method. Evaluation method's step are tagged on the model elements 


\subsection{Case Study Three: Course Registration System}

The notion of scenarios and tactics, and ATAM provide a general framework for modeling and analyzing NFRs, which is not applied in a security-specific case study by the authors or other researchers and practitioners. In this part, we apply the scenario and ATAM approach to an example system using the framework proposed in [27]. The example system is a simple Course Registration System. Students take or drop courses by choosing them from a course catalogue. Professors post final marks to the system and the system's admin edit courses. The privacy policies do not let the system's admin to view academic history of students, but professors have access to the marks of their own courses. Academic history of students is personal information and should not be disclosed to the other students.

The following sections provides the resulting artifacts and models using the framework in [27] and our proposed approach. The results are organized in two subsections. The first section gives the models and artifacts which express the security requirements and goals. Since the framework in [27] does not provide explicit means to model malicious actors and goals, the malicious goal model is presented in the first part. The second section provides the security solution models and trade-off analysis results.

\subsubsection{Scenarios and Goal Model}

In [27], quality requirements are expressed as scenarios. Table 4-1 summarizes security and performance scenarios for the example system. The approach of scenarios provides the means to express requirements by specifying a circumstance and expected behavior system in that context. The circumstance is described by a stimulus and its source. To express malicious actors' behavior, threat can be expressed by the stimulus 
whose source is the malicious actor. However, this approach does not provide means to express the impact and consequences of the attacks on other requirements.

Table 4-1. Scenarios for Course Registration case study

\begin{tabular}{|l|l|l|l|}
\hline & Scenario 1 & Scenario 1 & $\begin{array}{l}\text { Scenario 3 } \\
\text { (performance) }\end{array}$ \\
\hline Source & Jimmy's Friend & Jimmy's Friend & A transaction event \\
\hline Stimulus & $\begin{array}{l}\text { Tries to crack a } \\
\text { password }\end{array}$ & Tries to sniff transaction & Stochastic event \\
\hline Artifact & System data & $\begin{array}{l}\text { System data } \\
\text { communication }\end{array}$ & $\begin{array}{l}\text { Course Registration } \\
\text { System }\end{array}$ \\
\hline Environment & Open/online & Open/online & Normal mode \\
\hline Response & $\begin{array}{l}\text { Keep the password } \\
\text { secret }\end{array}$ & Protect confidential data & $\begin{array}{l}\text { Process the requested } \\
\text { transaction }\end{array}$ \\
\hline $\begin{array}{l}\text { Response } \\
\text { Measure }\end{array}$ & $\begin{array}{l}\text { Password is remained } \\
\text { secret }\end{array}$ & $\begin{array}{l}\text { No confidential data is iting } \\
\text { revealed }\end{array}$ & $\begin{array}{l}\text { Latency: User waiting } \\
\text { time should be less than } \\
1 \text { minute }\end{array}$ \\
\hline
\end{tabular}

The approach in [27] suggests selecting security design solutions in terms of tactics, but the framework does not provide means to model alternative tactics for each scenario. ATAM helps the designer evaluate each alternative. The evaluator creates a "Quality Attribute Utility" tree where utility is the expression of the overall goodness of the system. A utility tree begins with utility as the root node. Quality requirements are placed as children of utility. Scenarios are placed at leaves of the tree. Figure 4-17 gives the utility tree for the example system. The difficulty to achieve and importance of each scenario is ranked by high/medium/low scheme. 


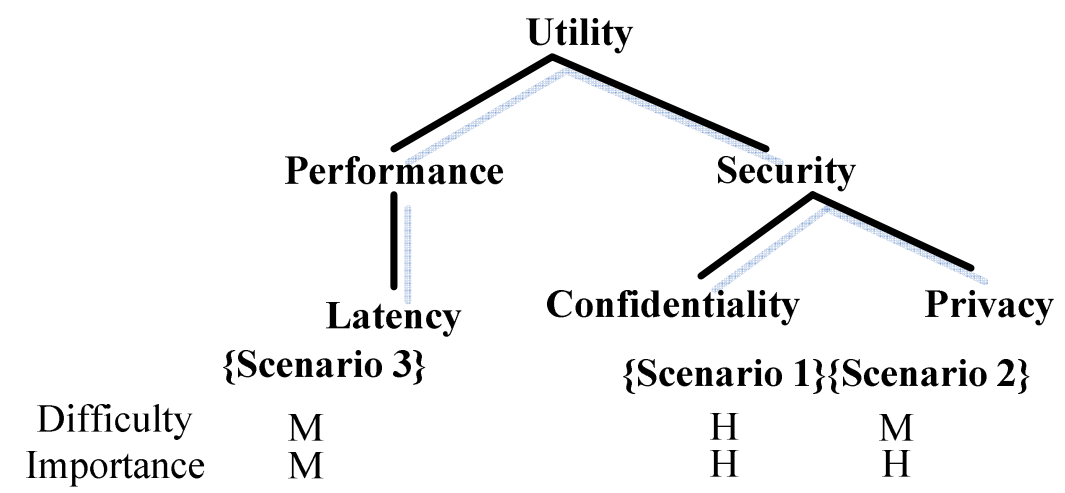

Figure 4-17. ATAM utility tree for the quality requirements of Course Registration System

Utility tree does not model the contributions of quality requirements on each other. Besides, this approach does not mode trade-offs among various quality scenarios. It does not provide the means to express the satisfaction degree of scenarios. One of the main weaknesses of utility tree approach is that it does not specify which actor imposes each scenario; therefore, the conflicts and trade-offs among goals are not expressed explicitly.

The goal model of the example system is given in Figure4-18 The focus of models is limited to Jimmy actor. The model includes the quality properties which we discussed earlier in form of softgoals. 


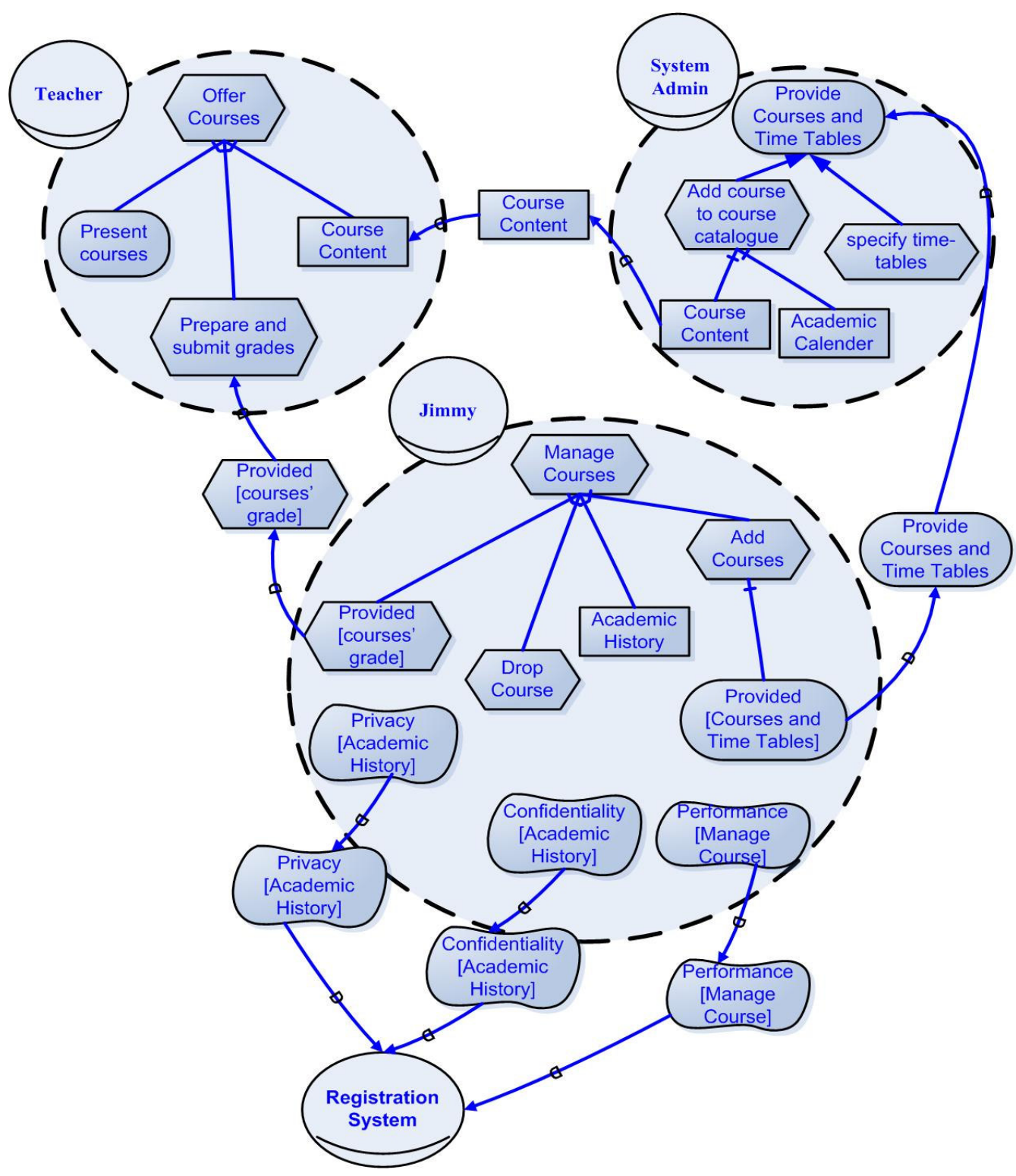

Figure 4-18. Goal model for the Course Registration System

Figure 4-19 gives the malicious goal model affecting Jimmy's goals. This model includes threats and security design alternatives. 


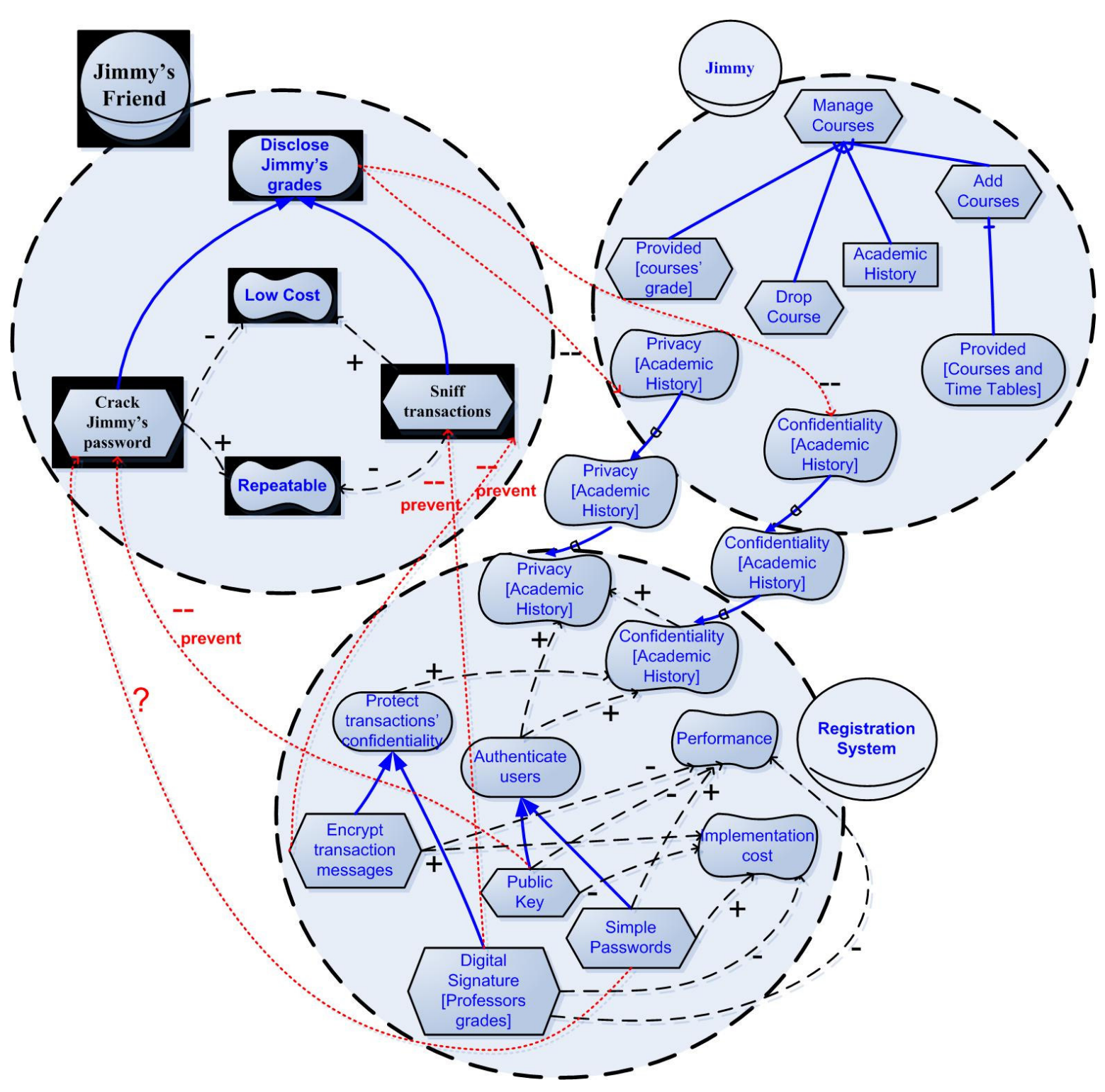

Figure 4-19. Security goals and trade-offs, modeled using the proposed notation. The impact and trade-offs of each security alternative are modeled using the contribution links

\subsubsection{Tactics, Security Goals and Trade-off Analysis}

Based on the ATAM recommendations, scenarios that have at least one high priority of importance or difficulty should be analyzed in more detail to examine if the decided tactics satisfy the scenario. The result is a "architectural approach analysis" table 
for each scenario. "Architectural approach analysis" table for the example system is presented in Table 4-2, which compares alternative tactics.

Table 4-2. Architectural approach analysis for Course Registration Case study

\begin{tabular}{|c|c|c|c|c|}
\hline Scenario 1 & \multicolumn{4}{|c|}{$\begin{array}{l}\text { Password should be remained secret when Jimmy's friend } \\
\text { tries to crack a password }\end{array}$} \\
\hline Tactic & Sensitivity & Trade-off & Risk & Nonrisks \\
\hline Simple password & performance & & & \\
\hline Public Key & & performance & & performance \\
\hline Scenario 2 & \multicolumn{4}{|c|}{$\begin{array}{l}\text { No confidential data would be revealed when Jimmy's } \\
\text { friend tries to sniff transaction }\end{array}$} \\
\hline Tactic & Sensitivity & Trade-off & Risk & Nonrisks \\
\hline $\begin{array}{ll}\text { Encrypt } & \text { transaction } \\
\text { messages } & \end{array}$ & & performance & per & \\
\hline Digital Signature & & performance & perfo & \\
\hline
\end{tabular}

The "architectural approach analysis" table does not provide means to express the impacts of each tactics on stimuli (attacks). Trade-offs among quality scenarios and tactics in the "Architectural Approach Analysis" table are indirect and implicit, since trade-off and risk points, instead of referring to quality scenarios, point to affected quality properties. Moreover, it does not capture the degree of sensitivity or trade-off between tactics and qualities. The analysis method does not provide any means to add evaluator's perception about degree of success of tactics or stimuli.

Figure 4-20 depicts the result of trade-off analysis using security goal model evaluation. The trade-offs of the chosen alternative and their impacts on threats are specified using the labels. The result of evaluation is that Encrypt transaction messages and Public Key mechanisms cause losing the performance. The implementation cost is not hurt by Public Key, it is but improved by Encrypt transaction messages, so there is a conflict on this goal. Both possible attacks would be controlled by these mechanisms. The performance goal is partially denied by the selected mechanisms. 


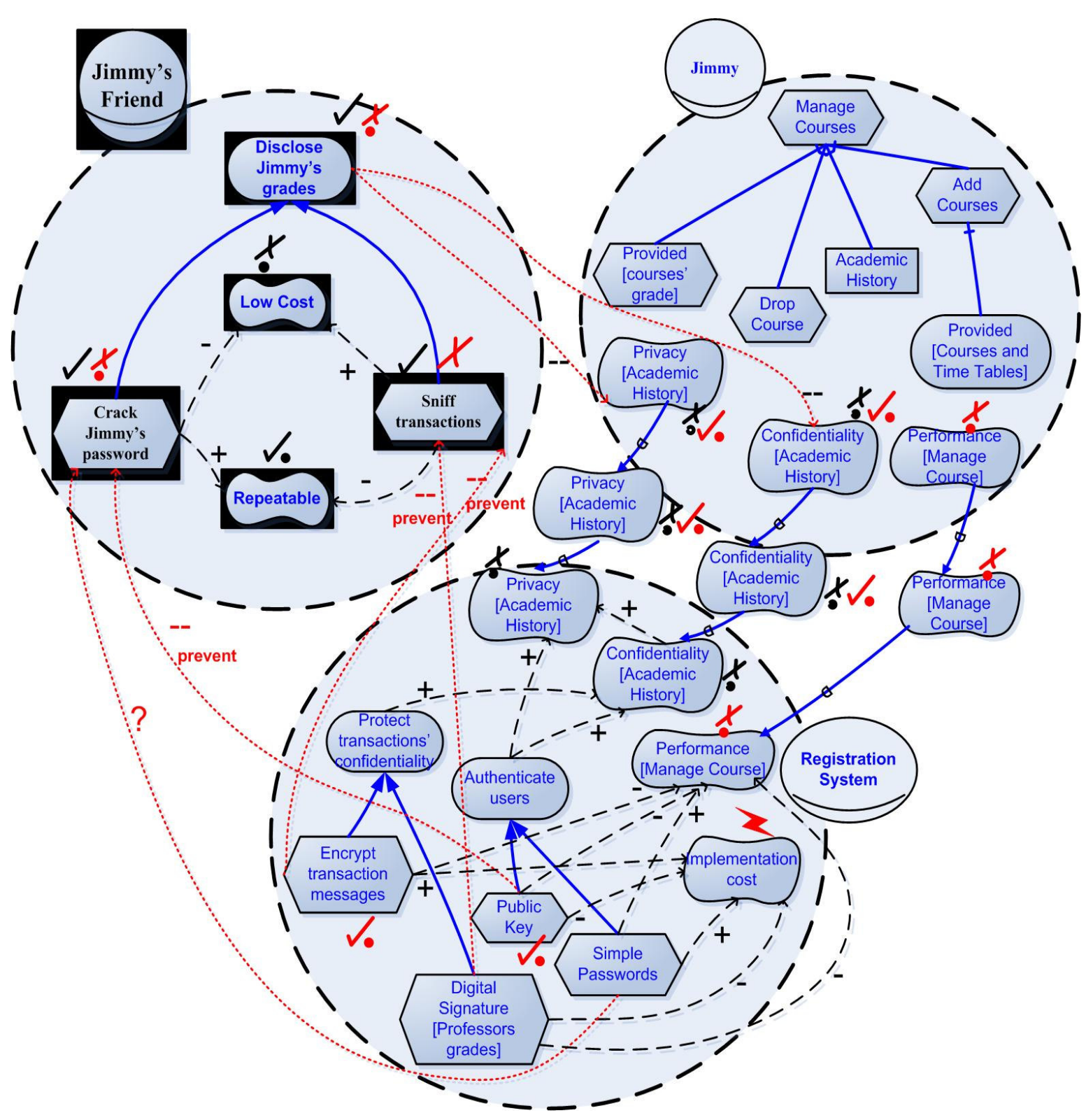

Figure 4-20. Security trade-off analysis, using the proposed evaluation method

The model in Figure 4-20 and the result of ATAM analysis in Table 4-2 conclude almost the same results. However, the goal model enables the designers to express the trade-off relations explicitly. Security goal models represent scenarios in terms of goals and softgoals, security scenario in terms of malicious goals, and tactics in terms of security mechanisms. Trade-offs among goals are explicitly modeled using contribution 
links. The goal model evaluation results are represented using satisfaction or denial labels, and represent the trade-off analysis results graphically and explicitly.

\subsection{Conclusions}

The case studies and comparison of resulting models revealed strong and weak points of the previous works and our proposed technique. The resulting models of the proposed method contain malicious goals and their contributions, which provide a malicious behavior perspective of the system. The alternative security mechanisms that each actor may select to protect his goals against the malicious behavior are added to the model, and trade-offs that mechanisms impose are incorporated into the models. Finally, the trade-off analysis evaluates the final impact of each alternative solution on other goals.

The main advantage of the proposed modeling technique is the explicit expressing of the concept of trade-offs among security mechanisms, threats and other goals of the actors. The goal model evaluation procedure and the resulting evaluation labels, which indicate the satisfaction or denial of the goals, provide an explicit way to represent the consequences of each alternative option, and help the designer explicitly make trade-offs based on an informed judgment. However, further studies need to be performed to evaluate the correctness of the decisions that designer make by employing various modeling and analyzing techniques.

The main issue related to the resulting models in $[22,25,26]$ is the lack of an explicit expression of the trade-offs among alternative solutions and goals of various actors. The resulting models in [22] do not capture the goals and intentions behind malicious behavior, and therefore, the models do not specify how a security alternative 
protects the goals of actors against the threats and attacks. Moreover, the resulting models in [22] do not specify which actor should perform a security mechanism, and the consequences of security mechanisms on goals of other actors are not modeled and analyzed.

The resulting models in $[25,26]$ do not express the trade-offs explicitly. In these approaches, the $i^{*}$ notation is extended with a new modeling notation for expressing the concept of threat, but the threats are not linked to malicious actors who impose the threat, which reduce the expressiveness and understandability of the models. Besides, the impact of security mechanisms on threats is not captured in the models. Therefore, the designer makes the trade-offs while the effectiveness of alternative solutions to protect the actors' goals against the threats is not considered.

The resulting artifacts of applying ATAM [27] for security trade-off analysis do not explicitly consider security-specific aspects such as threats and vulnerabilities; therefore, the artifacts do not capture the impact of threats on quality goals of actors. Besides, ATAM approach expresses the trade-offs among tactics and quality properties in a series of table, which comparing to graphical goal models, are less expressive and easy to understand. 


\section{The Software Security Trade-offs Knowledge}

\section{Base}

In the process of security trade-offs modeling and analyzing, which is described in Chapter three, designers rely on security knowledge gained from previous experiences, empirical studies, subjective knowledge, or common sense. Many recurring patterns of attacks and vulnerabilities have been identified by longtime software security practitioners [36]. On the other hand, software designers lack such knowledge about common vulnerabilities and attacks for different platforms, domains and applications, and security experts are expensive craftsman. McGrew asserts that the software security industry does not have enough security master craftsmen to effectively apprentice and train the world's software developers [37].

McGraw states that while critical software security knowledge and expertise can be compiled and shared widely [37], one of the challenges facing software security is the lack of an easily accessible common body of security knowledge [36]. Therefore, gathering and cataloguing reusable software security trade-offs knowledge in an organized, understandable and accessible catalogue would be beneficial for software designers. In this context, what are desirable characteristics of such a software security knowledge base $(\mathrm{KB})$ ? What knowledge items should be stored? What level of abstraction is appropriate for enabling the trade-off analysis? And finally, how should other formats of security knowledge be transformed and integrated into the KB?

In this chapter, first we survey various security knowledge sources considering and comparing properties and limitations of each one to extract properties of desired $\mathrm{KB}$ 
which includes required knowledge for making security trade-offs. We refer to such $\mathrm{KB}$ as software security trade-offs KB. Based on the captured criteria, we propose the schema of a $\mathrm{KB}$ for gathering and cataloguing security trade-offs knowledge in a reusable, structured, and searchable structure. We employ the extended $i^{*}$ notation for codifying the knowledge in the proposed KB. Finally, we discuss the units of knowledge of the proposed schema, and we discuss the requirements and criteria for transforming various formats of security knowledge to the proposed $\mathrm{KB}$ models.

However, this work is a preliminary analysis to obtain a $\mathrm{KB}$ schema, to complete the proposed goal oriented approach. This work needs a more extensive study drawing from broader perspectives including studying knowledge managements and knowledge engineering.

\subsection{Existing Security Knowledge Sources}

Designing security-critical systems requires knowledge and security expertise in various fields such as computer networks, operating systems, communication protocols, cryptography algorithms, and access control methods. Software designers usually lack such expertise and skills, and master craftsmen in security engineering field are typically expensive and usually unavailable. Therefore, designers require a source of knowledge about potential vulnerabilities and attacks, existing security safeguards, trade-offs among security mechanisms, and the impact of each one on security requirements and other design objective. Such knowledge could be acquired from various sources. We have identified four major categories of software security knowledge sources, based on the format and structure of knowledge sources, degree of formality, subject, and knowledge items: 
1. Security text knowledge: white papers such as [41], textbooks such as [32,33], guidelines like NIST [30], SANS [39], standards, checklists like SANS [39], and products' data sheets and resources provided by the vendors.

2. Security experiences' documents: collected cases for Case Based Reasoning, Design Rationale, and previous projects documents.

3. Security design patterns: security design patterns such as open group patterns [42], requirement-driven approach to design patterns [43], patterns for agent systems [44]

4. Structured catalogues and knowledge bases: web portal knowledge bases such as CERT [45], the proposed KB schema in [37], and NFR security catalogues [18]

\subsubsection{Security Textual Knowledge}

Security textbooks usually include a wide variety of knowledge ranging from high-level concepts to technical details. Security guidelines such as NIST guidelines [30, 46] contain similar type of knowledge in a more organized structure, which can be used as a practical development guide. Security checklists such as SANS security checklists provide designers with tips to verify security of the design solution. Security standards include practical security recommendations with emphasis on following proven solutions which the community of practitioners agrees on.

Generally, content of text knowledge sources is amorphous and unstructured, and security trade-offs are not often expressed explicitly; therefore, browsing, retrieving, manipulating, and expanding text knowledge sources is not efficient. Since text knowledge is represented informally and in natural languages, the designer needs to 
interpret the text content, which may lead to misinterpretation and inaccuracy of the captured information.

The unit of knowledge in text sources can be a sentence, a paragraph, a section or a chapter. Generally, the unit of knowledge of text sources is not fixed, and the smallest chunk of reusable knowledge might be a whole section or chapter. The text knowledge units are tightly coupled which burdens separating knowledge clusters from each other. These characteristics make the knowledge retrieval inefficient, since the designer needs to browse the whole content to extract the required information.

In particular, textbooks and guidelines are descriptive, abstract and theory oriented. Standards mostly address a specific context and domain. Checklists aim to remind the designer to follow certain recommendations and avoid pitfalls in technical and developmental details. In sum, text knowledge sources typically do not provide the designers with explicit knowledge required for analyzing security solutions and their trade-offs.

\subsubsection{Security Experiences' Documents}

Design rationale refers to the knowledge and reasoning behind a design decision. Design rationale is an explanation of why an artifact or some part of an artifact is designed the way it is [47]. Design rationale is used to record the rationale behind decisions, and therefore, trade-offs related to specific alternatives solutions are described explicitly. Documents of previous experiences, if gathered from numerous sources and projects, would contain recurring attack patterns and descriptions of successful or defeated solutions. 
However, documents of previous projects and experiences are limited to threats and safeguards which were analyzed and tested in a particular context in the past, and as a result, they lack generality and may not be applicable in a different context. Although both case-based reasoning and design rationale are rich sources of security design knowledge, they do not correspond with latest security attacks, recently discovered vulnerabilities, and new available security solutions. However, updating knowledge is an issue for other security knowledge sources as well. In addition, experience documents are not formally structured, and most of the mentioned issues related to informality of text-based knowledge sources are weak points of experience documents as well.

\subsubsection{Security Design Patterns}

Design patterns encapsulate experts' knowledge in form of proven solutions to common problems. Patterns are mostly structured text in form a three-part rule which expresses a relation between a certain context, a problem and a solution. One of the most comprehensive catalogues of security patterns is published by the Open Group [42]. In these catalogues, patterns are described based on the "Gang of four" format [48]. In this format, for each pattern, the intent, motivation, applicability, structure, consequences, known uses, and related patterns are specified. Figure 5-1 gives the elements of security design patterns of Open Group and correlation between the elements which has been explicitly mentioned in [42]. 


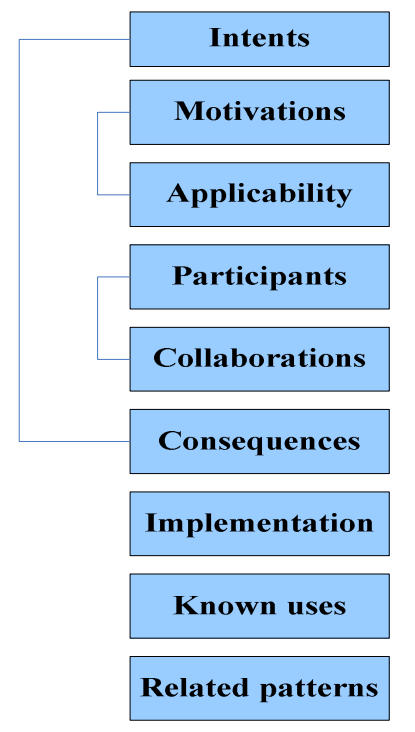

Figure 5-1. Elements of security design patterns of Open Group and correlation between the elements

Design patterns are more structured than other text format knowledge sources such as guidelines and design rationale. However, they are still in the format of informal text, and structure of design reasoning is not systematically organized [43]. Therefore, in [43], authors propose a requirement-driven approach to express design patterns to make the reasoning structure behind the design patterns explicit. This approach suggests modeling the impact of each design pattern to other NFRs and modeling how alternative solutions contribute differently to goals for characterizing patterns according to the NFRs. This goal oriented approach for expressing design patterns is extensively used in the current work to express security knowledge obtained from security patterns and other knowledge sources.

However, the proposed approach in [43] focuses on NFRs, and does not consider security patterns specifically, and as a result, does not model and catalogue vulnerabilities, threats, and security safeguards in the structure of patterns. The proposed approach lacks a systematic method for trade-off analysis among alternative 
design patterns and solutions. In addition, the resulting models may become large and complex, specially, if one integrates multiple design patterns into one goal model using the approach in [43] to analyze and compare alternative design patterns for a problem. The proposed approach does not provide procedures for browsing and extracting parts of the model. Finally, this work lacks a systematic method for transforming design patterns into the proposed NFR goal models in order to specify how elements of design patterns are mapped to different elements and parts of the NFR goal graph.

Authors in [44] suggest an approach for structuring security pattern for agent systems. The patterns are expressed in text accompanied with social dependencies model expressed by $i^{*}$ notation. The presented patterns express trade-offs explicitly in terms of “consequences". However, [44] does not provide a method for analyzing and evaluating the solution based on the trade-offs that patterns impose on other design objectives. Finally, the patterns are in form of informal texts, and most of the mentioned issues related to informality of text-based knowledge and text-based design pattern are weak points of these kinds of patterns as well.

\subsubsection{Structured Catalogues and Knowledge Bases}

The US-CERT Vulnerability Notes Database is a well-known web-based security catalogue which gathers security vulnerabilities in a searchable database. The notes are similar to alerts in which solutions may not be available for all the vulnerabilities [45]. US-CERT Vulnerability Notes catalogues vulnerabilities in various contexts, their impact and report reference. Generally, the structure of this knowledge source is limited to the vulnerabilities. SANS Top-20 lists of vulnerabilities is another web-based knowledge source which gathers the most critical vulnerabilities from the past year. 
Founded in 1901, National Institute of Standards and Technology (NIST) is a non-regulatory federal agency within the U.S. Computer Security Division (CSD) is one of eight divisions within NIST's Information Technology Laboratory. The mission of NIST's Computer Security Division is to improve information systems security. National Vulnerability Database (NVD), U.S. government repository of standards based vulnerability, is one of the web portals provided by NIST. NVD contains Common Vulnerabilities and Exposures (CVE) list that provides common names for publicly known information security vulnerabilities. NVD supports the Common Vulnerability Scoring System (CVSS) standard for all CVE vulnerabilities. The CVSS is an open standard for scoring vulnerabilities.

Figure 5-2 gives the schema of the described web-based knowledge catalogues and the relations among the catalogues. The emphasis of all these knowledge web portals is on gathering and cataloguing an updated list of vulnerabilities discovered for specific platforms, operating systems, protocols and products. These web portals rarely provide solutions for the vulnerabilities, and their application is limited to discovering new possible threats and maintaining system's security; therefore, they do not provide software designers the required knowledge to analyze the alternative security decisions and trade-offs each solution poses. 


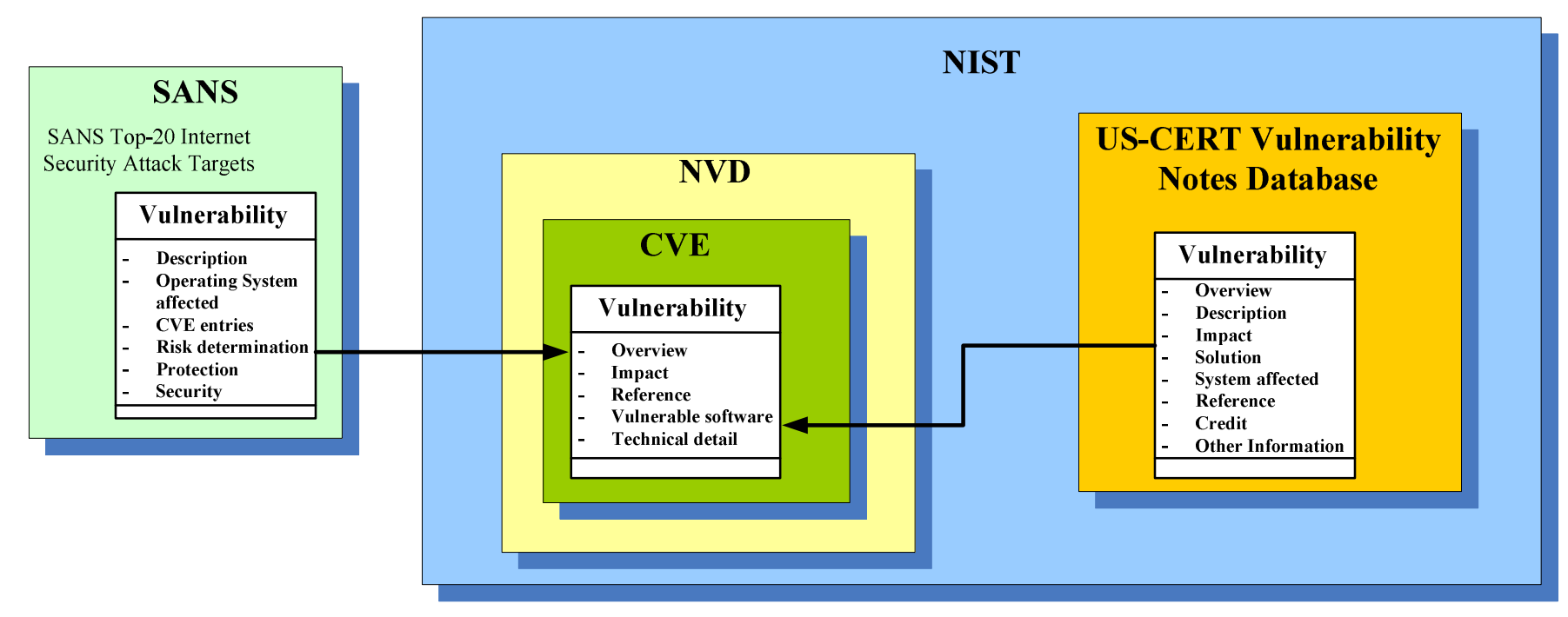

Figure 5-2. Relations between various knowledge web-portals

NFR security catalogues is another example of security knowledge catalogue. NFR is a framework for modeling and analyzing non-functional requirements [18]. In the NFR framework, non-functional requirements are expressed by notion of softgoals. Softgoals are goals that have no clear definition or criteria of satisfaction. In [18], the framework is employed to develop a Security Requirements Framework to provide the designers with a catalogue of security methods and trade-offs among them. It is suggested to gather a catalogue of security types such as confidentiality and integrity in the security types' catalogue diagram which shows different aspects of security.

Types are refined using decomposition, operationalization and argumentation. Decomposition methods can guide disambiguating and choosing appropriate notions of security from a rich, diversified set of security notions. Operationalization methods can guide the selection of specific security techniques. The interaction among security requirements and other non-functional requirements are modeled by correlations rules. 
Catalogues of correlations rules facilitate the systematic capture and reuse of knowledge of softgoal harmony and conflicts and trade-offs among Operationalization methods.

However, the NFR approach does not consider modeling threats, attacks, vulnerabilities, and assets. The methods in the catalogue of security operationalization are not related to security threats or vulnerabilities; therefore, the catalogue does not provide the designer with knowledge required for selecting security mechanisms for potential threats or discovered vulnerabilities.

Another example of software security $\mathrm{KB}$ is the schema a $\mathrm{KB}$ for software security proposed by Barnum and McGraw [37]. They identify seven knowledge catalogues: principles, guidelines, rules, attack patterns, historical risks, vulnerabilities, and exploits; and categorize them into three categories: prescriptive, diagnostic, and historical. Prescriptive knowledge category including principles, guidelines, and rules offers advice for what to do and what to avoid when developing security software. Diagnostic knowledge including attack patterns, exploits, and vulnerabilities help the designers to identify and deal with common problems that lead to security attacks. Historical knowledge includes the knowledge catalogue of historical risks.

The proposed schema of the software security KB in [37] does not consider cataloguing security mechanisms and assets corresponding to vulnerabilities and attacks. The most fundamental pieces of knowledge for analyzing the security trade-offs are the correlations among security mechanism, attacks and other requirements, and impact of security mechanisms on other NFRs and threats, but the proposed schema in [37] does not consider such knowledge items which explicitly express the trade-offs. 


\subsection{Knowledge Structure Properties}

This part discusses essential properties of a knowledge structure for trade-off analysis such as required knowledge items, proper knowledge unit, and suitable level of abstraction, formality, and domain dependency.

\subsubsection{Knowledge Items}

The foremost concern about a knowledge structure for software security trade-offs is the knowledge items it contains. As discussed in earlier chapters, security is about trade-offs among potential threats, existing safeguards and individual goals of multiple stakeholders. A KB to enable security trade-off analysis for designing software systems needs to contain knowledge items including:

1) Security mechanisms such as firewall, encryption, public key infrastructure.

2) Common attacks such as SQL injection, zero-day attack.

3) Common vulnerability points such as buffer overflow, password disclosure.

4) Assets such as data base records, communication channel.

Besides, relations among the concepts have significant role in trade-off analysis which most of knowledge sources lack:

5) Relation between attacks and safeguards

6) Relation between NFRs and safeguards

7) Relation among attacks, vulnerabilities and assets.

\subsubsection{General vs. Specific}

In [38] patterns are classified in four levels of abstraction: 1) concept 2) classes of pattern 3) patterns 4) examples. Level of abstraction of a KB is similar to this spectrum: 
from specific examples to high level concepts. We define the level of abstraction in four levels: 1) concepts 2) principles 3) patterns 4) examples. Examples include previous experiences such as employing a specific security mechanism in previous projects, or report of an attack. Patterns are formed from recurring examples by correlating an attack in a context and security solution which was employed to patch the vulnerability. Principles are proven rules that can be applied in every context, and at the highest level, the $\mathrm{KB}$ can be populated with general security concepts.

On the one hand, specific knowledge in a context is practical, since it is matched with the condition, and the designer does not need to interpret the knowledge and adjust it into the target context. On other hand, high level knowledge in general a context is more reusable, and can be applied in different conditions and circumstances [38], but the designer needs to fit the interpreted knowledge into the target context. This may reduce the accuracy of the extracted knowledge and efficiency of the applying the knowledge.

\subsubsection{Prescriptive vs. Descriptive}

McGraw asserts that prescriptive security knowledge offers advices for what to do and what to avoid when building secure software [37]. The prescriptive knowledge category provides the designers with alternative solutions to a problem. Descriptive knowledge contains high level concepts' explanations that can be used as guidelines. McGraw identifies diagnostic knowledge category, which helps practitioners recognize and deal with common problems that lead to security attacks. Attack patterns and common vulnerability points are catalogued as diagnostic knowledge category. 


\subsubsection{Formal vs. Informal}

Generally, formal knowledge representation facilitates systematic reasoning and analysis and automatic knowledge verification and validation. On the other hand, formal knowledge representation reduces the understandability of the stored and retrieved knowledge and is efficient as the knowledge communication language. Formally represented knowledge may need to be transformed to a less formal representation to be used in communication, modeling and analysis processes. In this way, expanding the KB is burdened with transforming implicit knowledge or text based documents to a formal representation which requires human's interpretation of source knowledge and translation to the target formal language.

\subsubsection{Domain-Independent vs. Domain-Specific}

Generally, the applicability boundary of domain-specific knowledge is limited to that domain. However, a high level abstract body of knowledge would not be applicable in real-world contexts. Although there are many common threats, attacks and vulnerabilities in various domains, there exist attack patterns particularly for each domain which requires particular security treatments. This requires that the KB contains software security trade-off knowledge for different domains.

\subsubsection{Units and Clusters of Knowledge}

One important concern to structure a body of knowledge is to define the smallest meaningful and reusable unit of knowledge in the cluster. It is desirable that the cluster of knowledge be composed of reusable independent subparts, which the whole cluster bears a more comprehensive piece of knowledge. Small units of knowledge increase the 
reusability, portability in different contexts, and understandability of the clusters, and facilitate reusing subparts of a body of knowledge. However, the units and subparts are more general than the whole cluster, and they need to be adjusted and integrated into the context, while the whole cluster fits in the target context with less adjustment.

\subsubsection{Usage of Knowledge}

Various knowledge sources have different usage in different development stage. We categorize usages as: 1) introductory to the security domain, 2) security requirements analysis, 3) design, 4) trade-off analysis, 5) test, and 6) monitor and maintenance.

Knowledge sources such textbooks are helpful to get familiar with security domain. In requirements analysis phase, more detailed and technical sources such as guidelines and standards are useful. In design phase, design patterns, standards and previous experience documents are useful knowledge sources. In test, monitor and maintenance phase, checklists and web portals provide required knowledge to constantly check the security of system design or operation.

Figure 5-3 gives a spectrum of generality and formality for various knowledge sources including the desired $\mathrm{KB}$. The generality spectrum ranges from very high level abstract form of security knowledge to technical details. Formality spectrum ranges from informal to semi-formal and formal knowledge representation. 


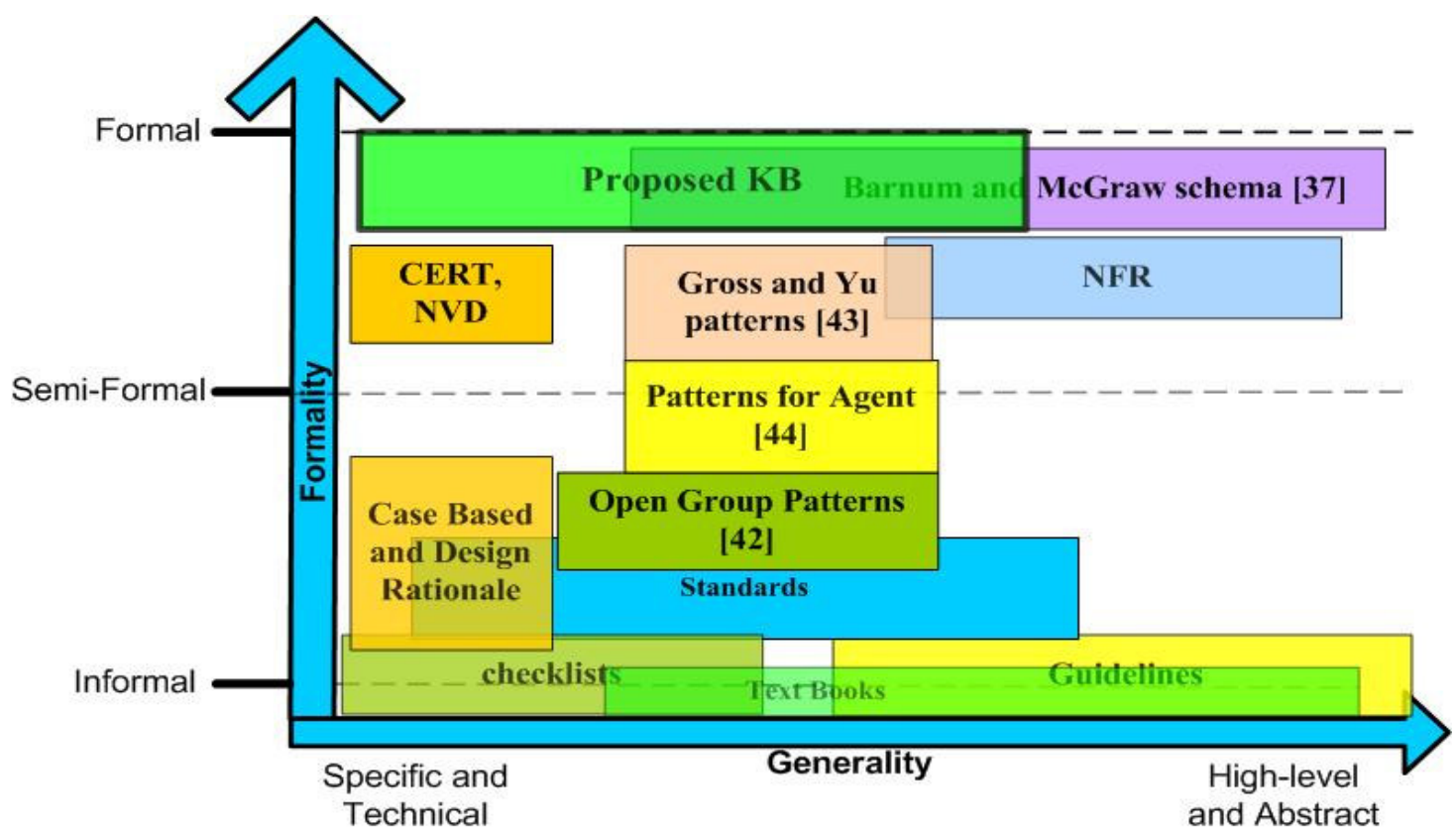

Figure 5-3. Spectrum of generality and formality of various security knowledge sources

Table 5-1 gives a comparison of various knowledge sources according to the discussed criteria, and usage of knowledge. 
Table 5-1. A comparison of various knowledge sources

\begin{tabular}{|c|c|c|c|c|c|c|c|c|c|c|c|c|c|c|c|c|c|c|c|c|c|c|c|c|c|c|c|}
\hline \multirow{2}{*}{\multicolumn{2}{|c|}{ Knowledge sources type }} & \multicolumn{4}{|c|}{ Generality } & \multirow[b]{2}{*}{ 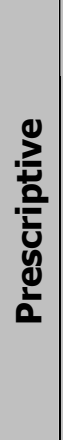 } & \multirow[b]{2}{*}{ 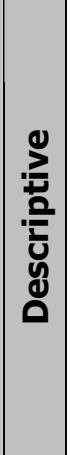 } & \multirow[b]{2}{*}{ 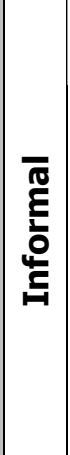 } & \multirow[b]{2}{*}{ 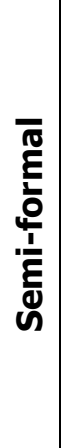 } & \multirow[b]{2}{*}{ 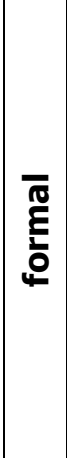 } & \multirow[b]{2}{*}{ 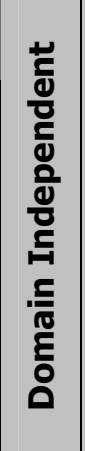 } & \multirow[b]{2}{*}{ 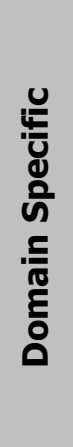 } & \multirow[b]{2}{*}{ 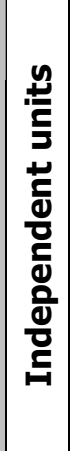 } & \multirow[b]{2}{*}{ 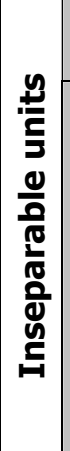 } & \multicolumn{7}{|c|}{ Knowledge Item } & \multicolumn{6}{|c|}{ Usage } \\
\hline & & 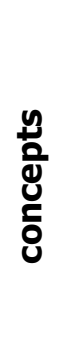 & $\begin{array}{l}\frac{y}{2} \\
\frac{2}{0} \\
\frac{2}{2}\end{array}$ & 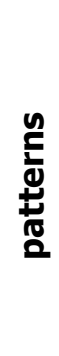 & $\frac{y}{\frac{y}{a}}$ & & & & & & & & & & 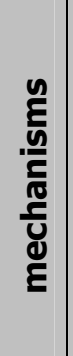 & $\begin{array}{l}\frac{y}{u} \\
\mathbb{E} \\
\frac{ \pm}{0}\end{array}$ & 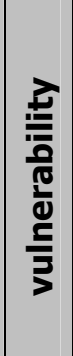 & 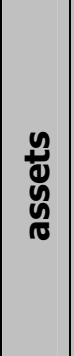 & 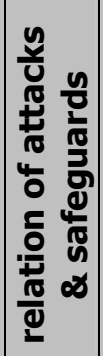 & 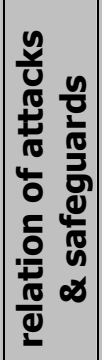 & 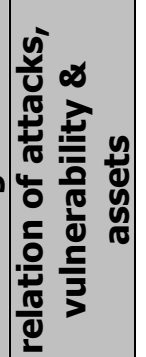 & 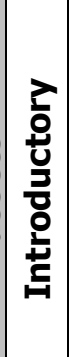 & 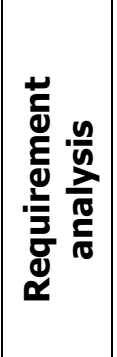 & $\begin{array}{l}\frac{5}{\bar{y}} \\
\frac{d}{0}\end{array}$ & 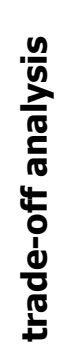 & 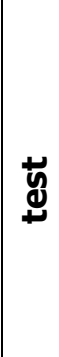 & 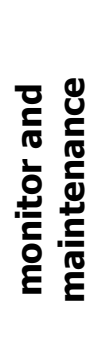 \\
\hline \multirow{4}{*}{$\begin{array}{c}\text { Textual } \\
\text { knowledge }\end{array}$} & Textbooks & $\sqrt{ }$ & $\sqrt{ }$ & $\sqrt{ }$ & $\sqrt{ }$ & $\sqrt{ }$ & $\sqrt{ }$ & $\sqrt{ }$ & $\times$ & $x$ & $\sqrt{ }$ & $\sqrt{ }$ & $x$ & $\sqrt{ }$ & $\sqrt{ }$ & $\sqrt{ }$ & $\sqrt{ }$ & $\times$ & $\sqrt{ }$ & $x$ & $x$ & $\sqrt{ }$ & $\sqrt{ }$ & $x$ & $x$ & $x$ & $x$ \\
\hline & $\begin{array}{c}\text { Guidelines (NIST, } \\
\text { SANS) }\end{array}$ & $\sqrt{ }$ & $\sqrt{ }$ & $x$ & $x$ & $\sqrt{ }$ & $\sqrt{ }$ & $\sqrt{ }$ & $\times$ & $x$ & $x$ & $\sqrt{ }$ & $x$ & $\sqrt{ }$ & $\sqrt{ }$ & $\sqrt{ }$ & $\sqrt{ }$ & $\sqrt{ }$ & $\sqrt{ }$ & $x$ & $\sqrt{ }$ & $\sqrt{ }$ & $\sqrt{ }$ & $\sqrt{ }$ & $\times$ & $x$ & $x$ \\
\hline & Standards & $\sqrt{ }$ & $\sqrt{ }$ & $\sqrt{ }$ & $x$ & $\sqrt{ }$ & $\sqrt{ }$ & $\sqrt{ }$ & $\times$ & $x$ & $\sqrt{ }$ & $\sqrt{ }$ & $x$ & $\sqrt{ }$ & $\sqrt{ }$ & $\sqrt{ }$ & $\sqrt{ }$ & $\sqrt{ }$ & $x$ & $x$ & $x$ & $\times$ & $\sqrt{ }$ & $\sqrt{ }$ & $x$ & $\sqrt{ }$ & $\sqrt{ }$ \\
\hline & Checklists & $\times$ & $\sqrt{ }$ & $x$ & $x$ & $\sqrt{ }$ & $x$ & $\sqrt{ }$ & $\times$ & $x$ & $\sqrt{ }$ & $\sqrt{ }$ & $\sqrt{ }$ & $x$ & $\sqrt{ }$ & $\sqrt{ }$ & $\sqrt{ }$ & $\sqrt{ }$ & $x$ & $x$ & $\times$ & $\times$ & $\sqrt{ }$ & $x$ & $x$ & $\sqrt{ }$ & $\sqrt{ }$ \\
\hline \multirow{2}{*}{$\begin{array}{l}\text { Experience } \\
\text { Documents }\end{array}$} & $\begin{array}{c}\text { Case Based } \\
\text { Reasoning }\end{array}$ & $\times$ & $\times$ & $x$ & $\sqrt{ }$ & $\sqrt{ }$ & $x$ & $\sqrt{ }$ & $\times$ & $x$ & $x$ & $\sqrt{ }$ & $x$ & $\sqrt{ }$ & $\sqrt{ }$ & $\sqrt{ }$ & $\sqrt{ }$ & $\sqrt{ }$ & $\sqrt{ }$ & $\sqrt{ }$ & $\sqrt{ }$ & $\times$ & $\sqrt{ }$ & $\sqrt{ }$ & $\sqrt{ }$ & $x$ & $x$ \\
\hline & Design Rationale & $\times$ & $\times$ & $x$ & $\sqrt{ }$ & $\sqrt{ }$ & $x$ & $\sqrt{ }$ & $\times$ & $x$ & $x$ & $\sqrt{ }$ & $x$ & $\sqrt{ }$ & $\sqrt{ }$ & $\sqrt{ }$ & $\sqrt{ }$ & $\sqrt{ }$ & $\sqrt{ }$ & $\sqrt{ }$ & $\sqrt{ }$ & $\times$ & $\sqrt{ }$ & $\sqrt{ }$ & $\sqrt{ }$ & $x$ & $x$ \\
\hline \multirow{3}{*}{$\begin{array}{l}\text { Design } \\
\text { Patterns }\end{array}$} & Open Group & $\times$ & $\times$ & $\sqrt{ }$ & $x$ & $\sqrt{ }$ & $x$ & $x$ & $\sqrt{ }$ & $x$ & $\sqrt{ }$ & $\times$ & $x$ & $\sqrt{ }$ & $\sqrt{ }$ & $\sqrt{ }$ & $\sqrt{ }$ & $\times$ & $\sqrt{ }$ & $\sqrt{ }$ & $x$ & $\times$ & $x$ & $\sqrt{ }$ & $\sqrt{ }$ & $x$ & $x$ \\
\hline & $\begin{array}{l}\text { Gross and Yu } \\
\text { patterns [43] }\end{array}$ & $\times$ & $\times$ & $\sqrt{ }$ & $\times$ & $\sqrt{ }$ & $\times$ & $x$ & $\sqrt{ }$ & $x$ & $\sqrt{ }$ & $x$ & $\sqrt{ }$ & $x$ & $\sqrt{ }$ & $\sqrt{ }$ & $\sqrt{ }$ & $x$ & $\sqrt{ }$ & $\sqrt{ }$ & $x$ & $\times$ & $x$ & $\sqrt{ }$ & $\sqrt{ }$ & $x$ & $x$ \\
\hline & $\begin{array}{c}\text { Patterns for agents } \\
{[44]}\end{array}$ & $\times$ & $\times$ & $\sqrt{ }$ & $x$ & $\sqrt{ }$ & $\times$ & $x$ & $\sqrt{1}$ & $x$ & $\sqrt{ }$ & $x$ & $\sqrt{ }$ & $x$ & $\sqrt{ }$ & $\sqrt{ }$ & $\sqrt{ }$ & $\times$ & $\sqrt{ }$ & $\sqrt{ }$ & $\times$ & $\times$ & $x$ & $\sqrt{ }$ & $\sqrt{ }$ & $x$ & $x$ \\
\hline \multirow{3}{*}{$\begin{array}{c}\text { Catalogues } \\
\text { and KB }\end{array}$} & Web portals & $\times$ & $x$ & $x$ & $\sqrt{ }$ & $\sqrt{ }$ & $\times$ & $x$ & $\sqrt{ }$ & $x$ & $x$ & $\sqrt{ }$ & $\sqrt{ }$ & $x$ & $\times$ & $\sqrt{ }$ & $\sqrt{ }$ & $\sqrt{ }$ & $x$ & $\times$ & $\times$ & $\times$ & $\sqrt{ }$ & $x$ & $x$ & $\sqrt{ }$ & $\sqrt{ }$ \\
\hline & $\begin{array}{c}\text { Barnum and McGrew } \\
\text { schema[37] }\end{array}$ & $\times$ & $\sqrt{ }$ & $\sqrt{ }$ & $\sqrt{ }$ & $\sqrt{ }$ & $\sqrt{ }$ & $x$ & $x$ & $\sqrt{ }$ & $\sqrt{ }$ & $\sqrt{ }$ & $\sqrt{ }$ & $x$ & $\times$ & $\sqrt{ }$ & $\sqrt{ }$ & $x$ & $x$ & $x$ & $x$ & $x$ & $x$ & $\sqrt{ }$ & $\sqrt{ }$ & $x$ & $x$ \\
\hline & NFR & $\times$ & $\times$ & $\sqrt{ }$ & $x$ & $\sqrt{ }$ & $x$ & $x$ & $\sqrt{1}$ & $x$ & $\sqrt{ }$ & $\times$ & $\sqrt{ }$ & $x$ & $\sqrt{ }$ & $\times$ & $x$ & $\times$ & $x$ & $\sqrt{ }$ & $x$ & $\times$ & $x$ & $\sqrt{ }$ & $\sqrt{ }$ & $x$ & $x$ \\
\hline
\end{tabular}




\subsection{The Software Security Trade-offs KB}

Security issues are ultimately about trade-offs among alternative security mechanisms, security goals, other NFRs and goals of multiple stakeholders. This section presents the proposed schema of the software security trade-offs KB. The elements of the schema are captured from knowledge items which we discussed earlier in section 5.2.1. The proposed schema provides a structure to capture and catalogue prescriptive and formal security knowledge in form of independent reusable units of examples and patterns for a specific domain or general conditions.

\subsubsection{The KB Schema}

One essential feature of the security trade-offs $\mathrm{KB}$ is to catalogue NFR goals (besides security goals) and the contributions of security decisions to the other goals. As mentioned earlier in section 5.2.1, the KB should catalogue common threats and attacks, vulnerabilities, assets, security mechanisms, impact of mechanisms on attacks, and contributions of solutions to the goals.

Figure 5-4 presents the schema of the proposed $\mathrm{KB}$ which contains the required knowledge items to decide on alternative security design solutions. The extended $i^{*}$ notation which was introduced in Chapter three is adopted as the graphical codification of knowledge items in the proposed schema. Detail descriptions of mapping between elements of the schema and modeling constructs of the graphical notation is provided in Chapter three as well. The meta-model of the proposed notation and the schema of the $\mathrm{KB}$ are identical, since the gathered knowledge in the $\mathrm{KB}$ should cover all required 
modeling elements which the designer may required to employ in the security trade-offs models.

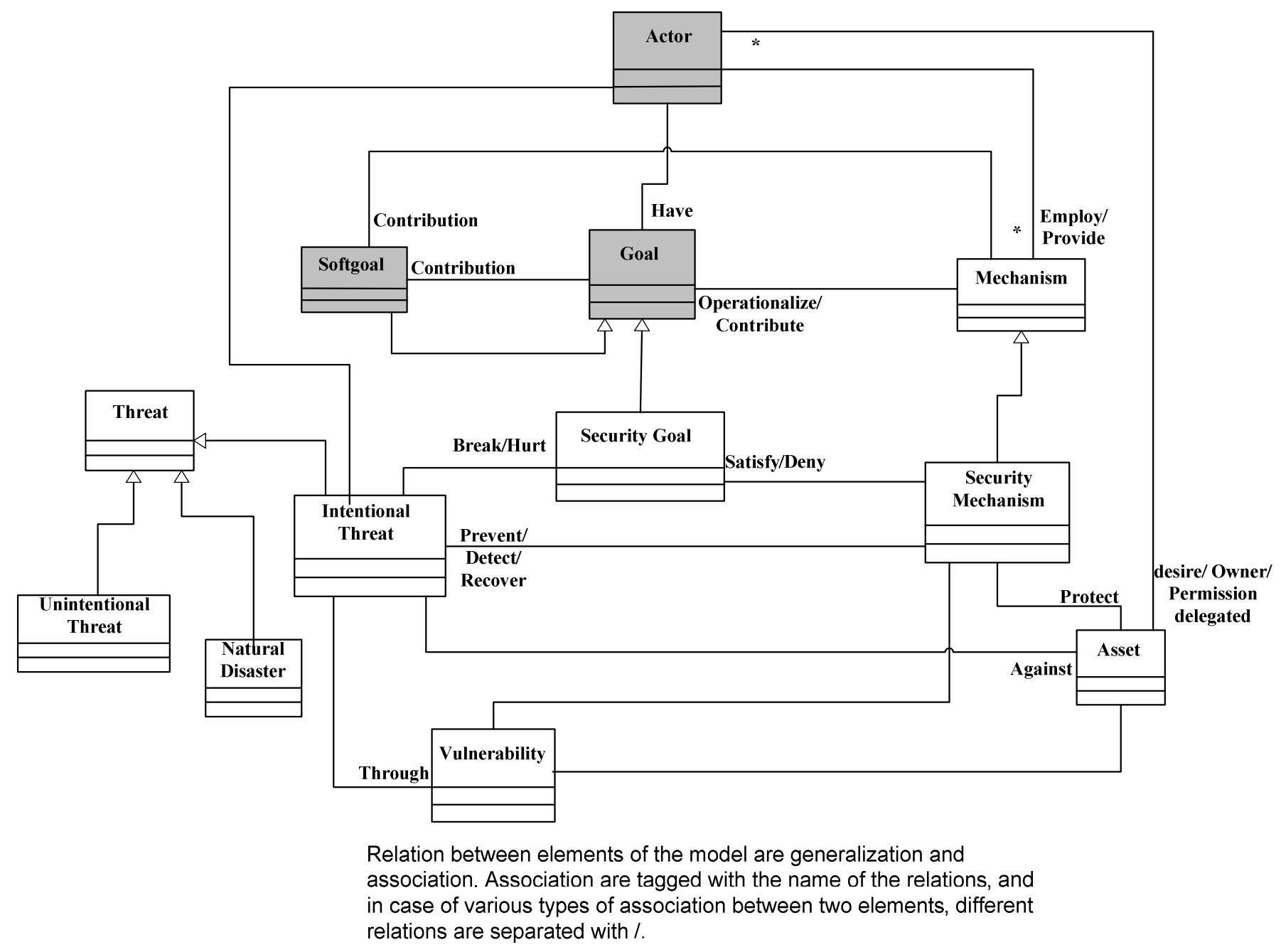

Figure 5-4. The knowledge base schema. The shadowed elements are taken from $i^{*}$ notation

\subsubsection{Unit of Knowledge}

Generally, particular elements of the KB schema such as a single vulnerability point are not knowledge items, since it is not attached to a context. A body of related information in a context that can be reused and applied in the real world condition is counted as knowledge; therefore, a combination of the elements of the schema forms the 
unit of knowledge. In this regard, what combinations of elements of the schema are reusable and meaningful that can be counted as knowledge unit?

This depends on the usage of knowledge, navigation path through the body of knowledge, and type of the questions which the knowledge user inquires from the KB to decide on security trade-offs. To analyze security trade-offs designers, developers and maintainers may have various inquiries:

1. What are alternative solutions to prevent/detect/recover a particular threat or vulnerability?

2. What attacks threaten the system through a particular vulnerability?

3. What assets (or services) are at risk of a particular attack? (And vice versa, what attacks threaten a particular asset?)

4. What are the impacts and consequences of applying a particular security solution on goals and attacks?

Figure 5-5 summarizes various knowledge units which are retrieved from the KB according to each of the above mentioned queries. Figure 5-5-A, bears the requested knowledge of the fourth query. The user navigates this body of knowledge to find the consequences of applying a specific security mechanism on softgoals and potential threats. 

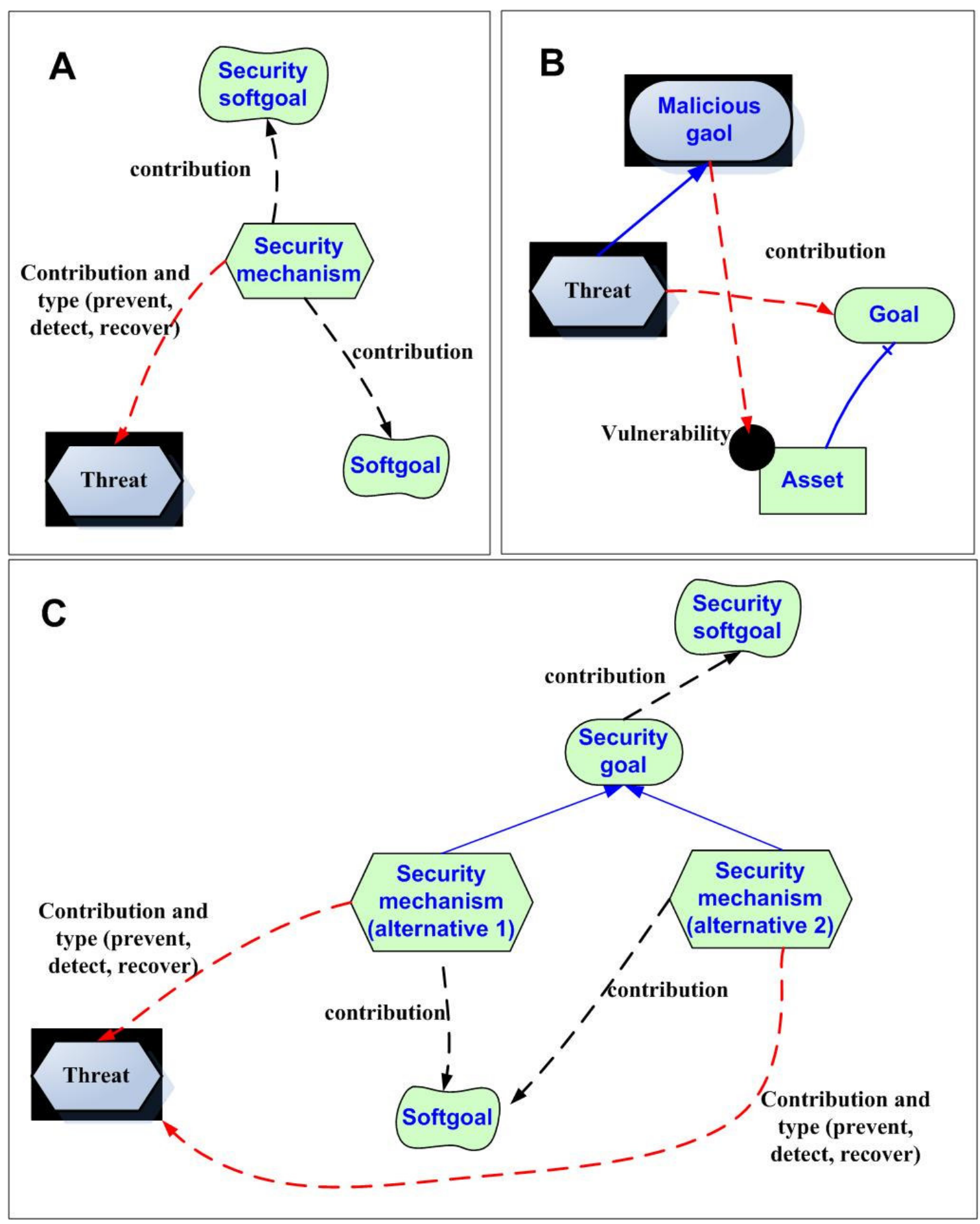

Figure 5-5. Various knowledge units of the schema 
Figure 5-6 gives the elements of the schema which shapes the knowledge unit of Figure 5-5-A.

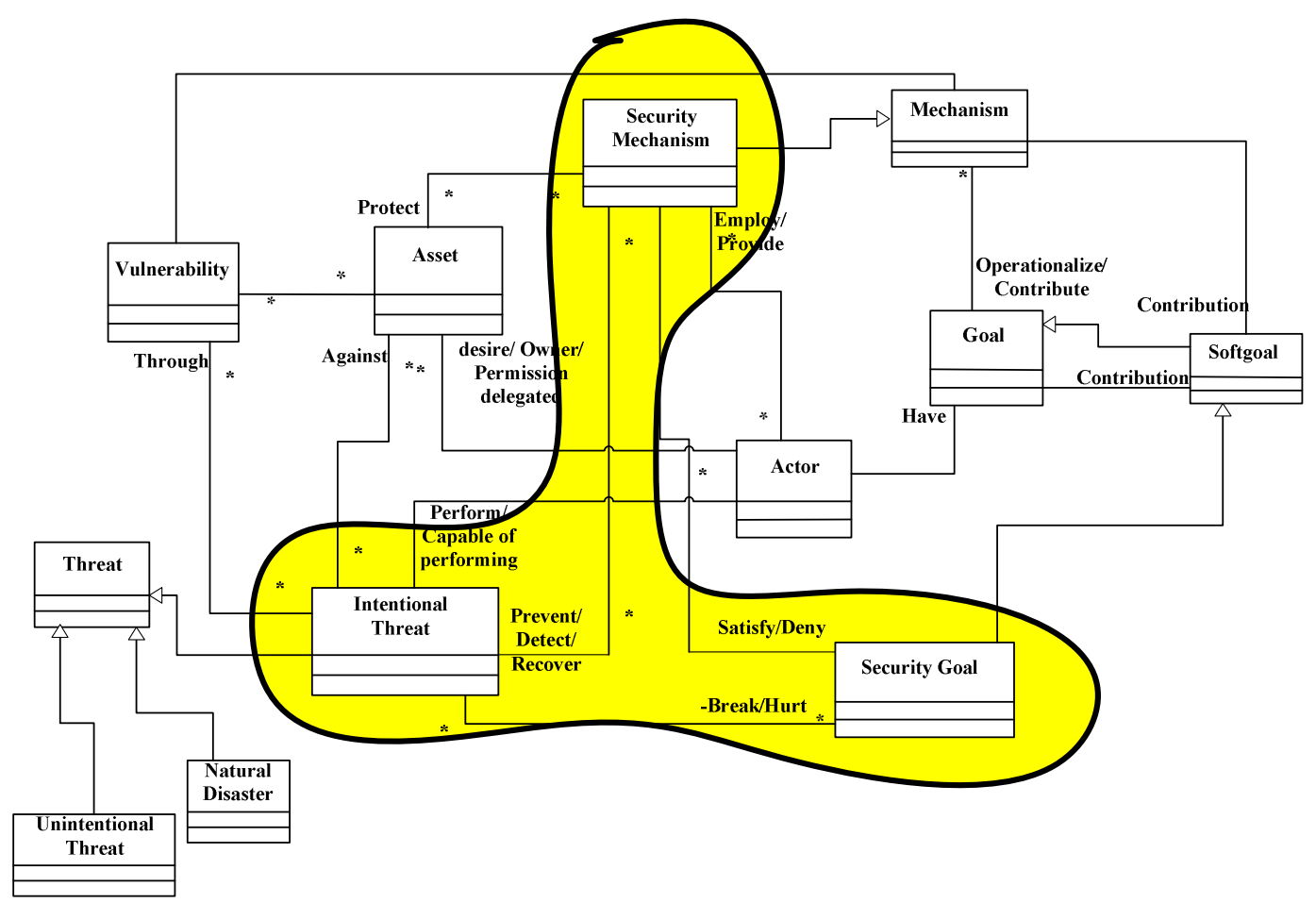

Figure 5-6. Elements of the schema which shapes the knowledge unit of Figure 5-5-A and Figure 5-5-C are located in the highlighted area.

Figure 5-5-C, which is a more elaborated knowledge unit of Figure 5-5-A, represents the unit of knowledge which contains security solutions that prevent/detect/recover an attack. The impact of each security solution on softgoals and malicious goals are expressed by contribution links. This knowledge unit bears the requested knowledge of the first query. This unit can be navigated from a particular attack to find alternative security solutions which can prevent/detect/recover the attack. Figure 5-5 gives the elements of the schema which shapes the knowledge unit of Figure $5-5-\mathrm{C}$ 
Figure 5-7 gives a sample knowledge unit of Figure 5-5-C. The sample knowledge unit gives two alternative ways to achieve prevent password guessing goal. The unit includes the contribution of each mechanism on log-in convenience softgoal and the malicious task of automated password guessing which usually employed software tools to check all possible passwords [46].

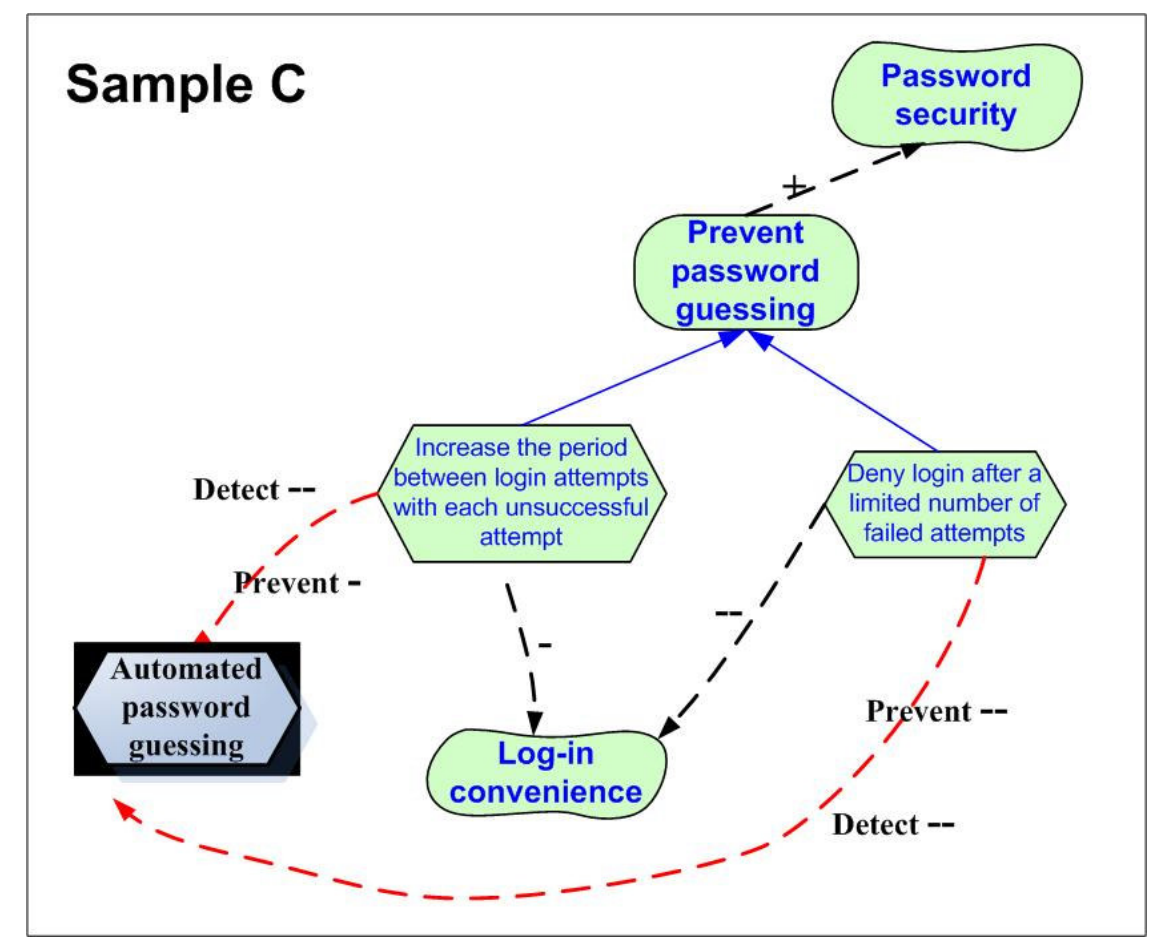

Figure 5-7. A sample knowledge unit

Figure 5-5-B depicts the unit of knowledge which the KB returns for the second and third queries. This knowledge unit includes common vulnerability of an asset and corresponding attack which may happen through that vulnerability. The user may start navigating this piece of knowledge from a particular attack to find the corresponding target assets and the vulnerabilities which can be abused as a backdoor to exploit the attack. The navigation can be initiated from a particular asset and its vulnerability points 
to find the potential attacks which threaten the system through the vulnerability. Figure 5-

8 gives the elements of the schema which shapes the knowledge unit of Figure 5-5-B.

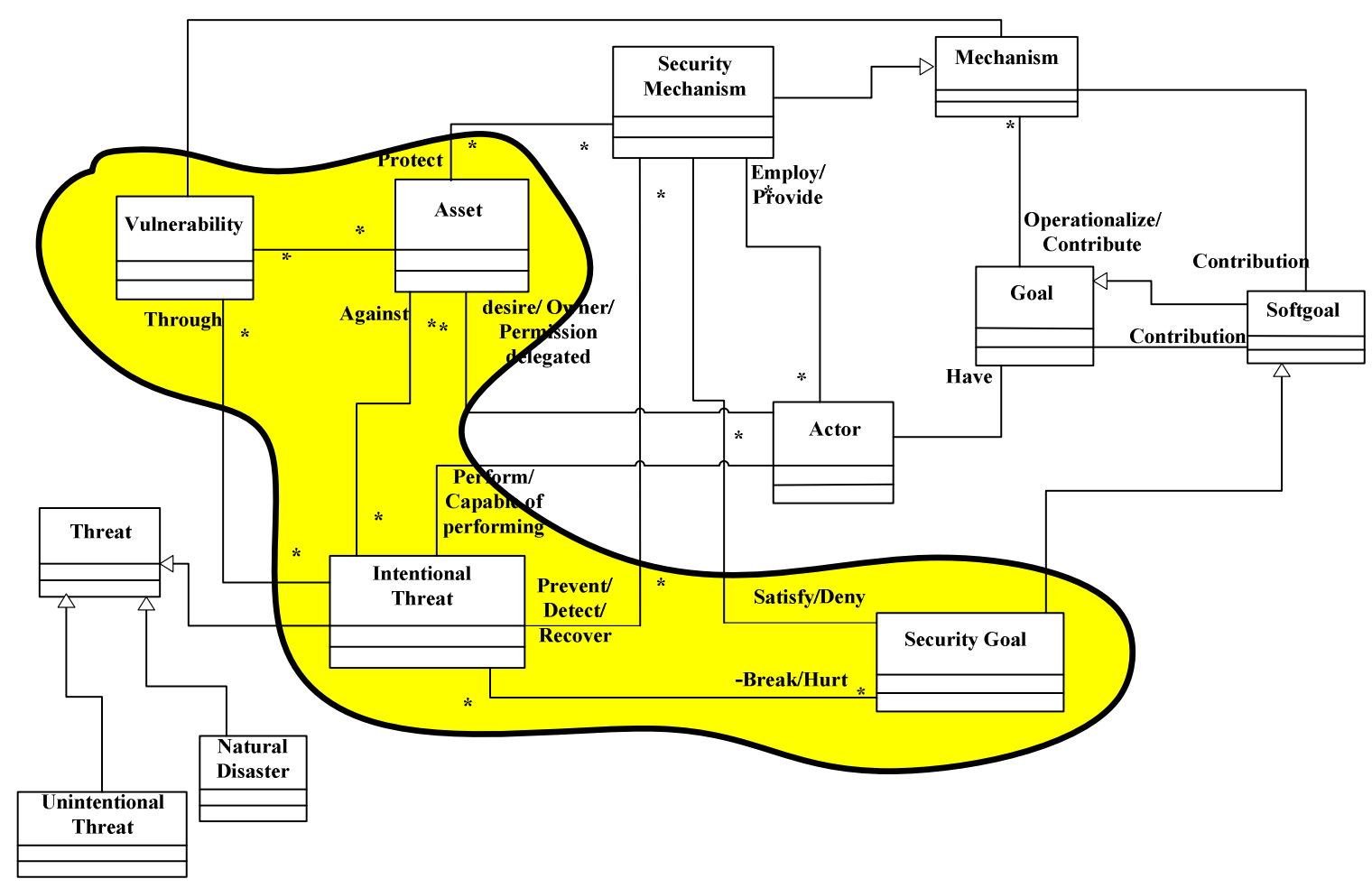

Figure 5-8. Elements of the schema which shapes the knowledge unit of Figure 5-5-B are located in the highlighted area.

Figure 5-9 gives a sample of this knowledge unit. Email has increasingly been used as a means for sending binary files in the form of attachments for day-to-day collaboration in many organizations. However, e-mail attachments can be a critical backdoor for zero-day attacks, since many forms of malware, including viruses, worms and spywares infect workstations through e-mail attachments [46]. 


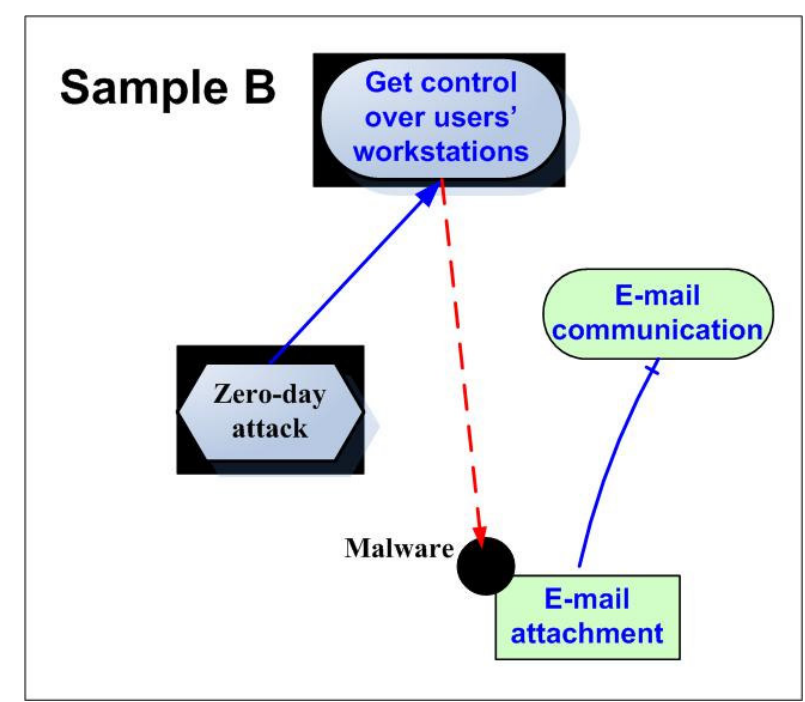

Figure 5-9. A sample for knowledge unit of Figure 5-5-B

However, based on the usage, the unit of knowledge can be a larger chunk of elements, and contains an attack, the related vulnerability, and security mechanism which control the attack.

\subsubsection{Populating the KB}

The process of transforming different knowledge formats to the $i^{*}$ goal oriented trade-offs models is not a straightforward process, since transformation is based on the human interpretation of the source knowledge. The human transformer needs to decide what parts of the source knowledge should be directly integrated into the KB models, which parts should be excluded, and what necessary elements to maintain the integrity and meaningfulness of the resulting models are missed in the source knowledge and need to be added to the knowledge models. However, if the transformer needs to add extra elements to the perceived information, the resulting models may not reflect the knowledge source. In this context, traceability of source knowledge to resulting models is 
an important concern. It is essential to denote elements and parts of the knowledge model to the corresponding parts of the source knowledge.

In addition, transforming process should be repeatable, and in case of duplicated transformations the resulting models should be consistent. Using a Wiki approach to populate the $\mathrm{KB}$, incorrect interpretations or inaccurate knowledge models may be corrected and completed by other transformers. Finally, populating the KB is not limited to transforming source knowledge to models, and the resulting $\mathrm{KB}$ should be expandable and modifiable.

To transform knowledge from one format to the other, the human transformer needs a defined map between meta-model of source and destination knowledge formats. However, most of the knowledge sources such as textbooks and standards are amorphous and unstructured. Therefore, in order to transform the text format knowledge to the knowledge models of the proposed schema, required knowledge items should be inquired from the text. For example:

- What are the usages of the mechanism?

- What goals can be achieved by employing the mechanism?

- How these goals may be threatened?

- How the attacks work?

- What are the malicious goals behind a security attack? Why an entity may threaten the goals?

- What is the impact of the attacks on the goals of the mechanism?

- What alternative security mechanisms may prevent or detect these threats, or the system can be recovered in case of successful attacks? 
- How the security mechanisms work? (What goals should be achieved, and what tasks and recourses are required to set up the security mechanism?

- Who is responsible to achieve these goals and perform the tasks?

- What are the consequences and impacts of applying these mechanisms on goals and threats?

The next chapter provides two example cases of transforming a text based body of knowledge and knowledge of a security design pattern to the elements of the proposed knowledge schema. 


\section{Example Cases of Populating the Knowledge Base}

This chapter gives two examples of transforming knowledge from a source to the proposed $\mathrm{KB}$ schema, and populating the $\mathrm{KB}$ with the elicited knowledge. To illustrate the transformation process, we transform software security knowledge from two knowledge formats, and model the interpreted knowledge in the structure of the proposed KB schema, using the extensions to $i^{*}$ as the graphical codification. The KB is populated by parts of a book chapter from [32], and a security design pattern from [42].

"Security engineering: a guide to building dependable distributed systems" [32], by Ross Anderson is a well known book in security engineering domain. The knowledge presented in third chapter of this book is transformed to the $\mathrm{KB}$ schema for illustrating how an amorphous and unstructured body of knowledge is transformed to the proposed knowledge format.

Security design patterns of Open Group are also well known design patterns in security domain. We describe the mapping between structure of these design patterns and the proposed KB schema. "Secure Communication" pattern from this group is transformed to the $\mathrm{KB}$ models as an illustration of how a semi-structured body of knowledge can be transformed to the proposed knowledge models.

\subsection{Transforming a Book Chapter to the KB Models}

Chapter three of [32] is dedicated to passwords, since passwords are one of the most important and yet difficult design problems in many secure system. Anderson discusses psychological and technical issues of using passwords as authentication and 
access control means, and a large part of this chapter gives technical protection mechanisms of passwords.

The text-based body of knowledge is not structured, and as a result, required knowledge should be extracted, interpreted, and transformed to the target models. To transform amorphous knowledge of the textbook chapter into the $\mathrm{i}^{*}$ goal model, the human transformer needs to extract the answer of the questions which we introduced in the previous chapter:

- What are the usages of passwords?

- The answer to this question forms the high level goals in the model.

- What goals can be achieved by employing passwords?

$\circ$ The answer to this question specifies the goal refinements.

- How these goals may be threatened?

- The answer to this question provides the possible attacks against the actors' goals.

- How the attacks work?

- The answer to this question specifies the required malicious goals and tasks to perform an attack.

- What are the malicious goals behind a security attack? Why an entity may threaten the goals?

- The answer to this question specifies the malicious actors' high level goals.

- What is the impact of the attacks on the goals of the passwords' owners? 
- The answer to this question denotes the contribution links between attacks and goals of other actors.

- What alternative security mechanisms may prevent or detect these threats, or the system can be recovered in case of successful attacks?

- The answer to this question provides the alternative goals and tasks which can satisfy security goals and protect the actors' goals from malicious behavior.

- How the security mechanisms work? (What goals should be achieved, and what tasks and recourses are required to set up the security mechanism?)

$\circ$ The answer to this question gives the goal refinements and task decomposition for the security goals and tasks.

- Who is responsible to achieve these goals and perform the tasks?

- The answer to this question illustrates the actors to which the security goals and tasks should be assigned.

- What are the consequences and impacts of applying these mechanisms on goals and threats?

- The answer to this question denotes the contribution links between security mechanisms and malicious tasks and goals.

Asking the listed questions, we summarized the text knowledge of this chapter into a model. Figure 6-1 gives the resulting knowledge model extracted from the book chapter. For example, the model presents that the attacker has a number of alternative ways to steal a password. Each alternative has effects on other attackers' softgoals. 


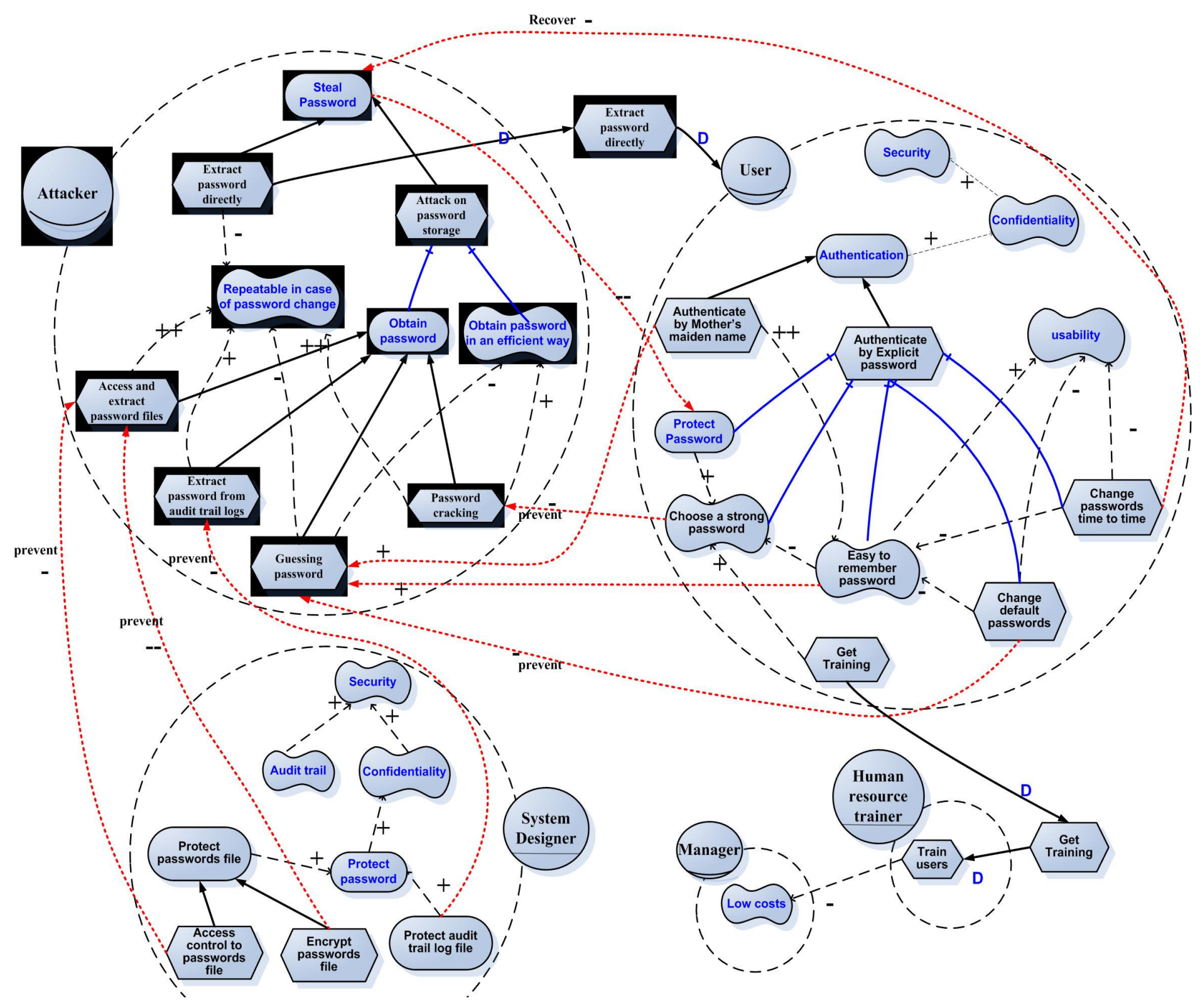

Figure 6-1. Resulting knowledge model from transforming a textbook chapter into the KB schema

Knowledge of threats, security solutions and the trade-offs they impose is not structured and organized in the textbook; therefore, human interpretation is required to extract the relevant items from the amorphous body of textual knowledge. In this context, 
1. what parts of the body of source knowledge should be directly incorporated into the knowledge models?

2. What parts should be changed and adjusted into the models?

3. What new items need to be added to make the resulting knowledge model intelligible yet consistent with the source knowledge?

Figure 6-2 specifies how various elements of knowledge model are obtained from the textbook. Shadowed areas indicate added or interpreted knowledge items, and other non-shadowed elements are obtained directly from the textbook. For example, the textbook introduces Authenticate by Mother's maiden name as a method for user authentication, but the impact of this method on the softgoal of Easy to remember [password] is not explicitly mentioned; therefore, the contribution link between the softgoal and the method is interpreted from the chapter discussions on psychological issues related to passwords.

In some cases, to ensure the significance of the resulting model, new elements are required to be added to the knowledge model. For instance, the textbook points to necessity of users' training to Choose strong passwords, but the book content does not consider that users' training requires Human resource trainer, which contributed negatively on low cost softgoal of the Manager. Hence, these items are elicited and added to improve the integrity and completeness of the resulting models. Eliciting new knowledge items indicates incompleteness and insufficiency of the sources knowledge. Further research is required to study how new knowledge items should be systematically elicited, and how 
additional knowledge sources can be employed to cover the incompleteness of one source.

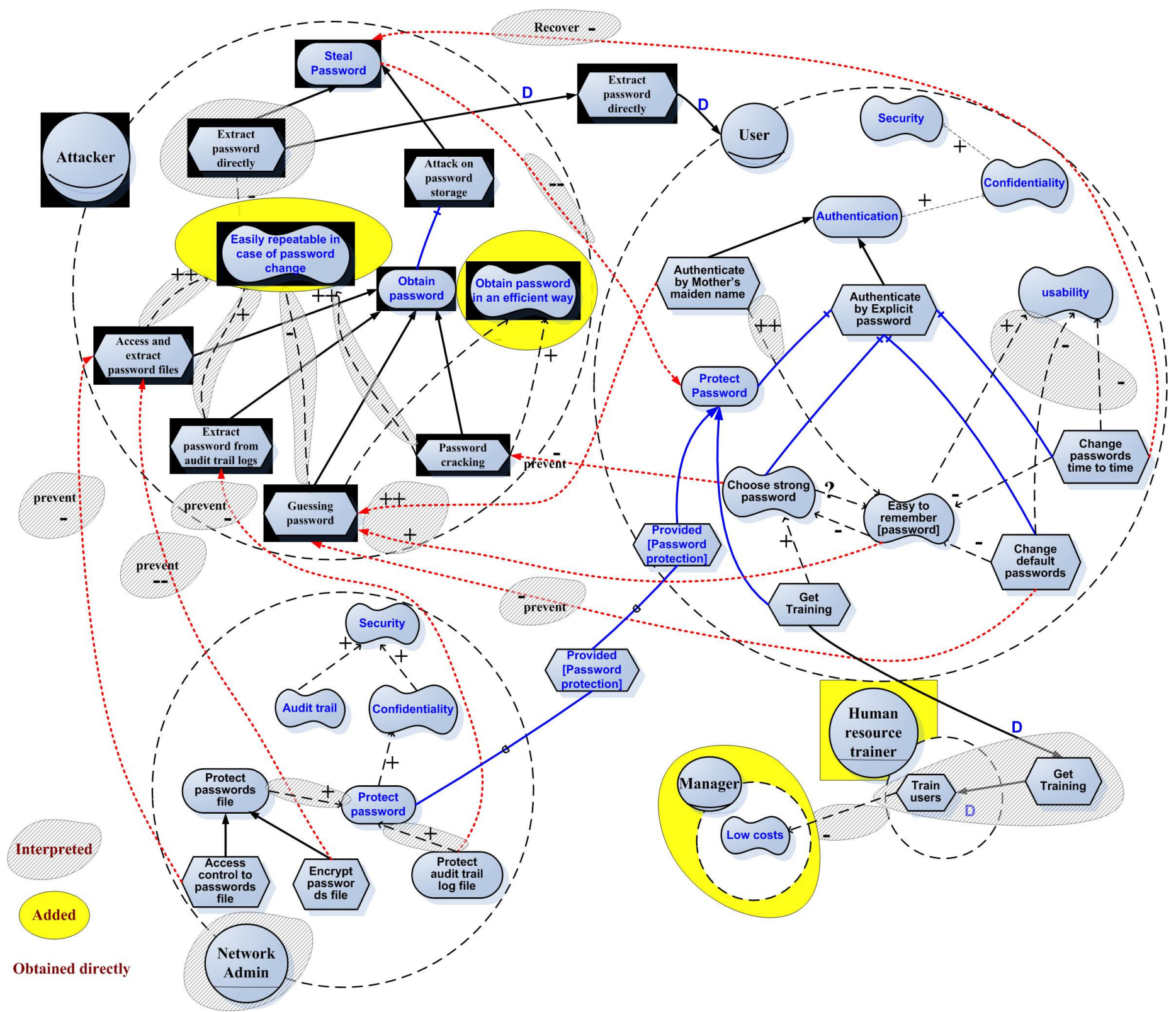

Figure 6-2. Resulting knowledge models with highlights which indicate how textbook content are transformed to $\mathrm{KB}$ schema elements 


\subsubsection{Extracting Knowledge Units from the Knowledge Chunk}

Previous section gives an example of transforming the knowledge gathered in a chapter of a textbook to our proposed knowledge models. The questions that arise here are: what are the knowledge units in the body of knowledge shown in Figure 6-2? And how the knowledge units can be extracted from the knowledge chunk? As discussed in the previous chapter, the knowledge units correspond to the questions that the designer asks to make trade-offs.

For example, one may ask "what are the alternative solutions to prevent Access and extract password files threat?" The result of this inquiry retrieves the unit of knowledge highlighted in Figure 6-3.

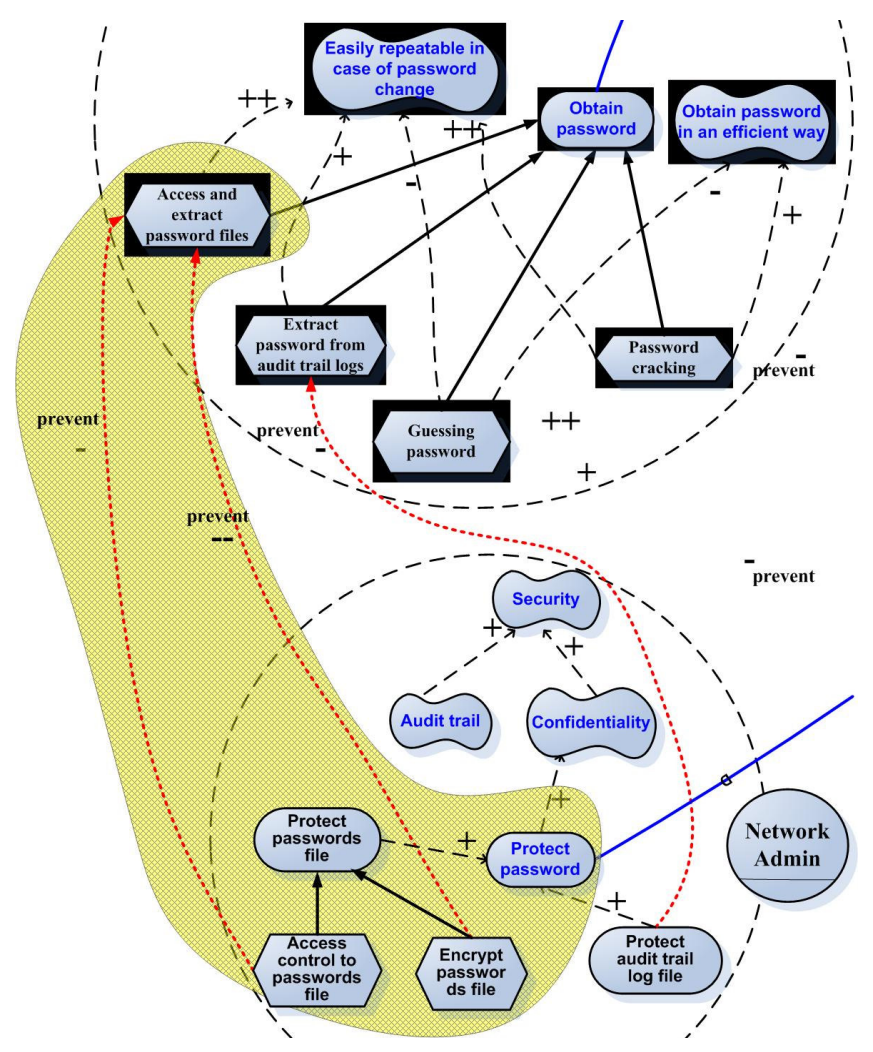

Figure 6-3. A unit of knowledge from the whole knowledge chunk of Figure 6-2, which corresponds to the knowledge unit of Figure 5-5-C. The retrieved knowledge unit is shadowed in the knowledge chunk. 
Each knowledge unit is elicited according to a design question and an algorithm to extract the unit from the chunk. The algorithm that extracts this unit of knowledge (Figure 5-5-C) from the knowledge chunk works as below:

- Locate the threat which the query refers to

- Extract all prevent contribution links which point to the threat

- Extract all alternative tasks (or goals) that the contribution links originate from

- Extract any higher goal or softgoals which alternatives contribute to

\subsection{Mapping Security Design Patterns to the KB Models}

A security design pattern is a semi-structured body of knowledge, and transforming a pattern to a new knowledge format requires less human interpretation than textual knowledge requires. However, the knowledge items in the design pattern should be mapped to elements of the schema. In this part, the knowledge provided in "security communication" pattern [42] is transformed and populated into to the proposed KB schema.

In this context, the main concern is how to map the knowledge items of the design patterns to the elements of the KB schema. The structure of security design patterns in [42] consists of: Intent, Motivation, Applicability, Participants, Collaborations, Consequences, Implementation, Known Uses, and Related Patterns:

- Intents are mapped to the high level goals in the target knowledge model.

- Motivations are scenarios of design problems which are expressed in terms of high level malicious goals and refinement of threats and attacks. 
- Applicability of patterns are expressed using goals related to the domain.

- Participants are be mapped to the actors.

- Collaborations are expressed in terms of actors' goals.

- Consequences in design patterns express the trade-offs which are modeled using $i^{*}$ contributions links.

- Implementation part in the design pattern can be used to refine the goals, tasks, and resources related to security mechanisms.

- Known uses are examples of the patterns found in real systems from different domains.

- Related patterns part contains related patterns and differences.

Figure 6-4 summarizes the mapping between structure of design patterns and the KB schema elements. The proposed schema of the KB does not directly consider "related patterns" and "known uses" as knowledge items in the schema. However, using meansend relation of $i^{*}$ notation, alternative mechanisms for achieving a goal can be expressed. 


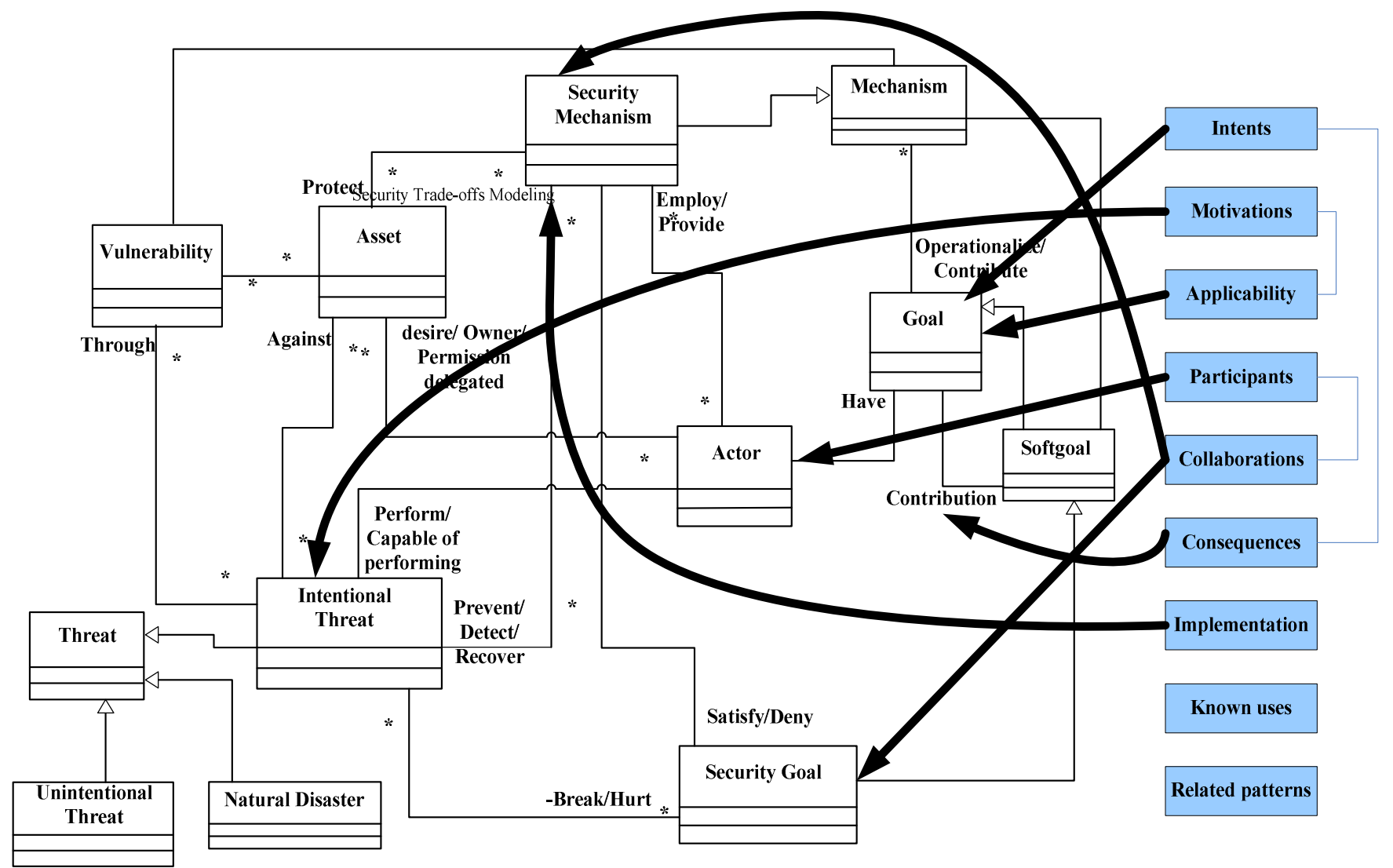

Figure 6-4. Mapping between elements of security design patterns and elements of the proposed KB schema

Figure 6-5 gives the resulting knowledge model of the "security communication" pattern. 


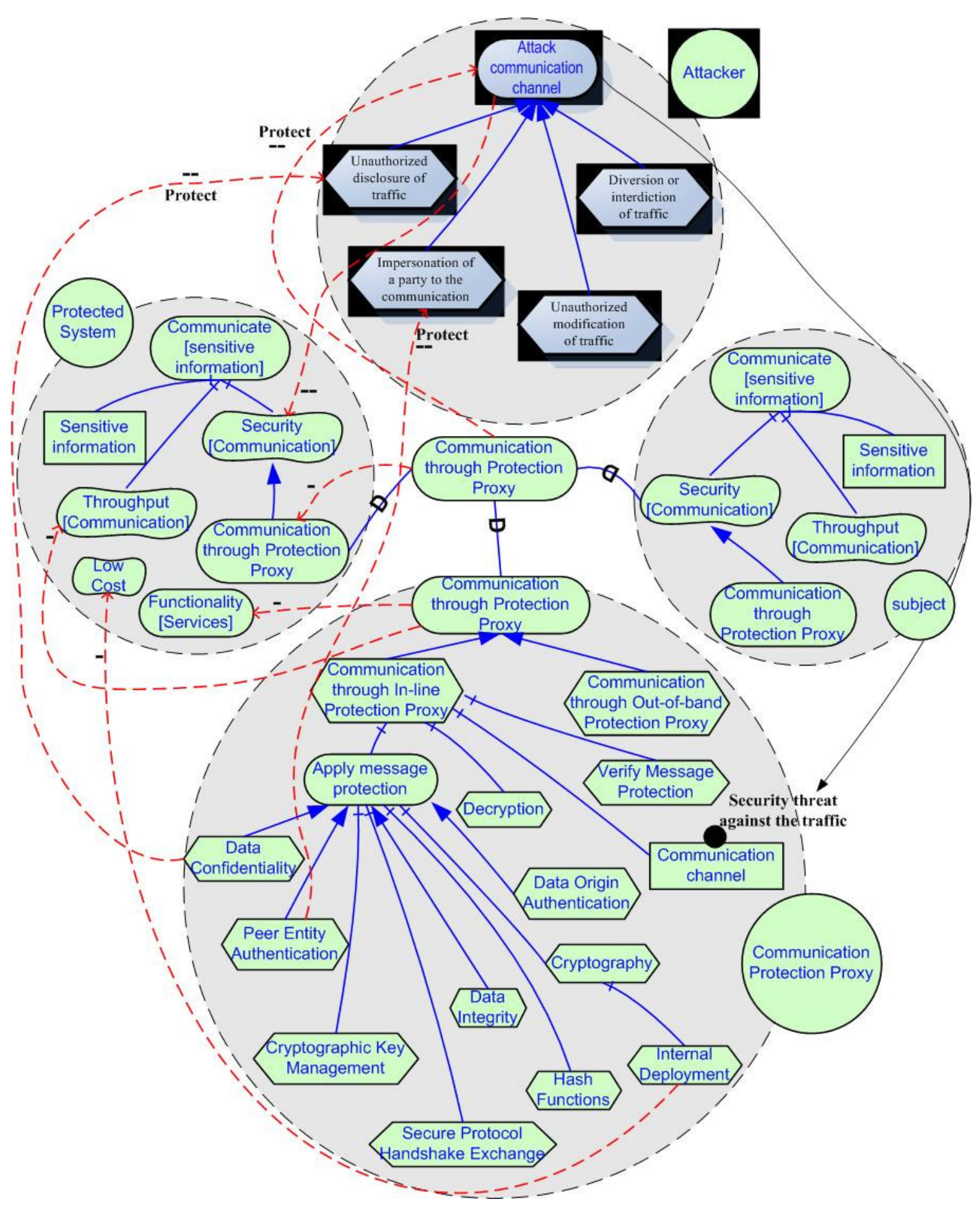

Figure 6-5. Resulting knowledge models from transforming "secure communication" design patterns to the proposed KB schema 
Transforming the pattern to the goal-oriented knowledge model reveals a number of incomplete and inaccurate parts in the pattern:

- The relation between threats in motivation part of the pattern and the security mechanisms in implementation part is not explicitly and directly specified. Although the pattern discusses what security mechanisms protect the system against various kinds of threats, the mentioned threats are not directly related to the threats introduced as the motivation.

- The consequence part of the pattern does not provide the designer with the degree of the impact of the pattern's solution on other goals; therefore, the contribution links in the resulting model need to be tagged by interpretation of the modeler.

- Moreover, the type of the impact of security mechanisms on threats is limited to "protect", while the security mechanisms may detect or prevent a threat, or recover the system after occurrence of an attack.

- The collaboration part of the pattern introduces two different approaches to communication proxy: inline proxy and out-of-band proxy, but the consequences of each one are not specified.

Figure 6-6 gives the knowledge model including guides which traces each knowledge element to the source of the knowledge item in the pattern. 


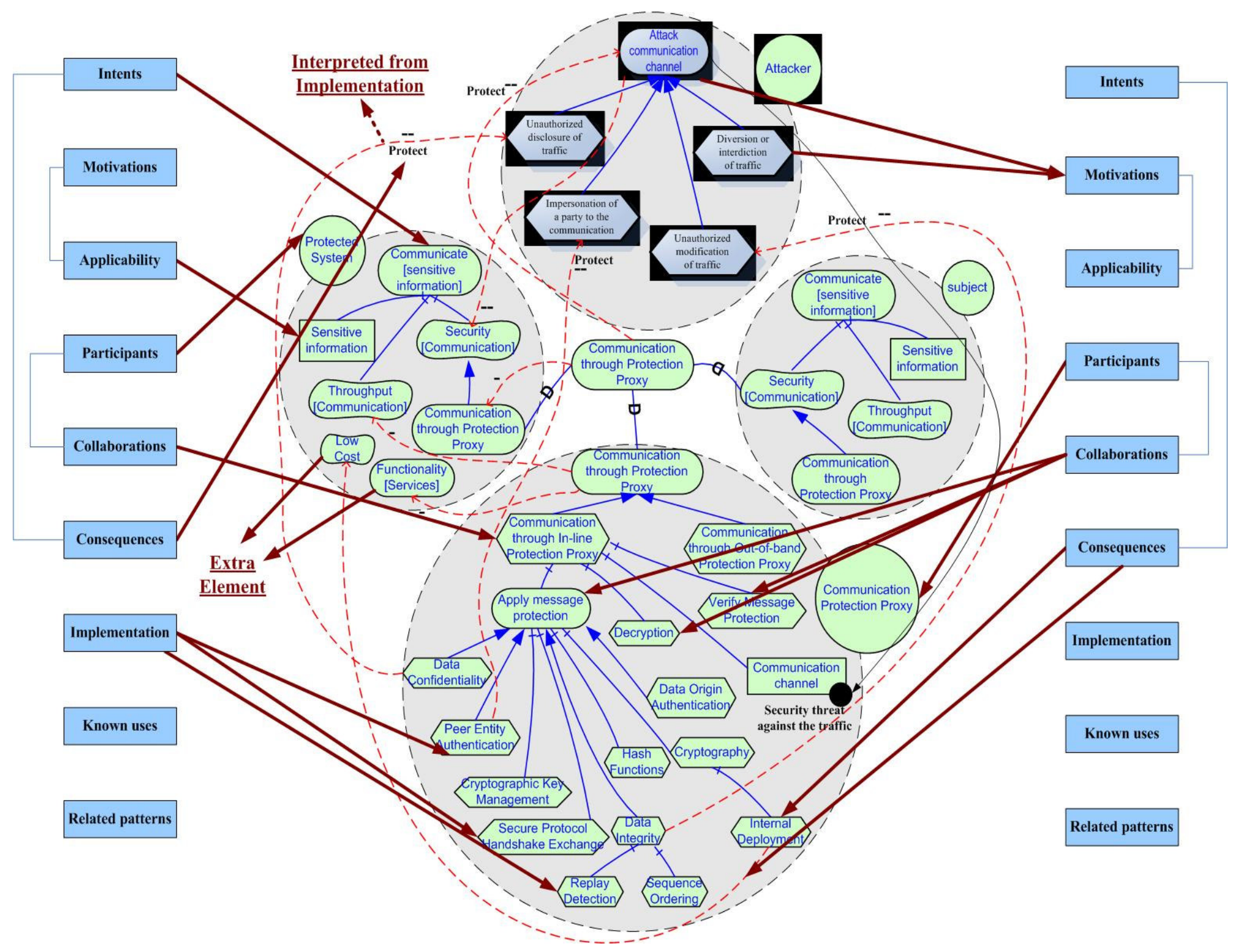

Figure 6-6. Resulting knowledge models with tags which indicate the map between security design patterns and KB schema elements

\subsubsection{Extracting Knowledge Units from the Knowledge Chunk}

This part discusses the other knowledge unit that can be extracted from the chunk of knowledge. One may ask "what attacks threaten the system through Security threat against the traffic vulnerability?" The result of this inquiry retrieves the unit of knowledge 
highlighted in Figure 6-7, which correspond to the introduced knowledge unit in Figure 5-5-B.

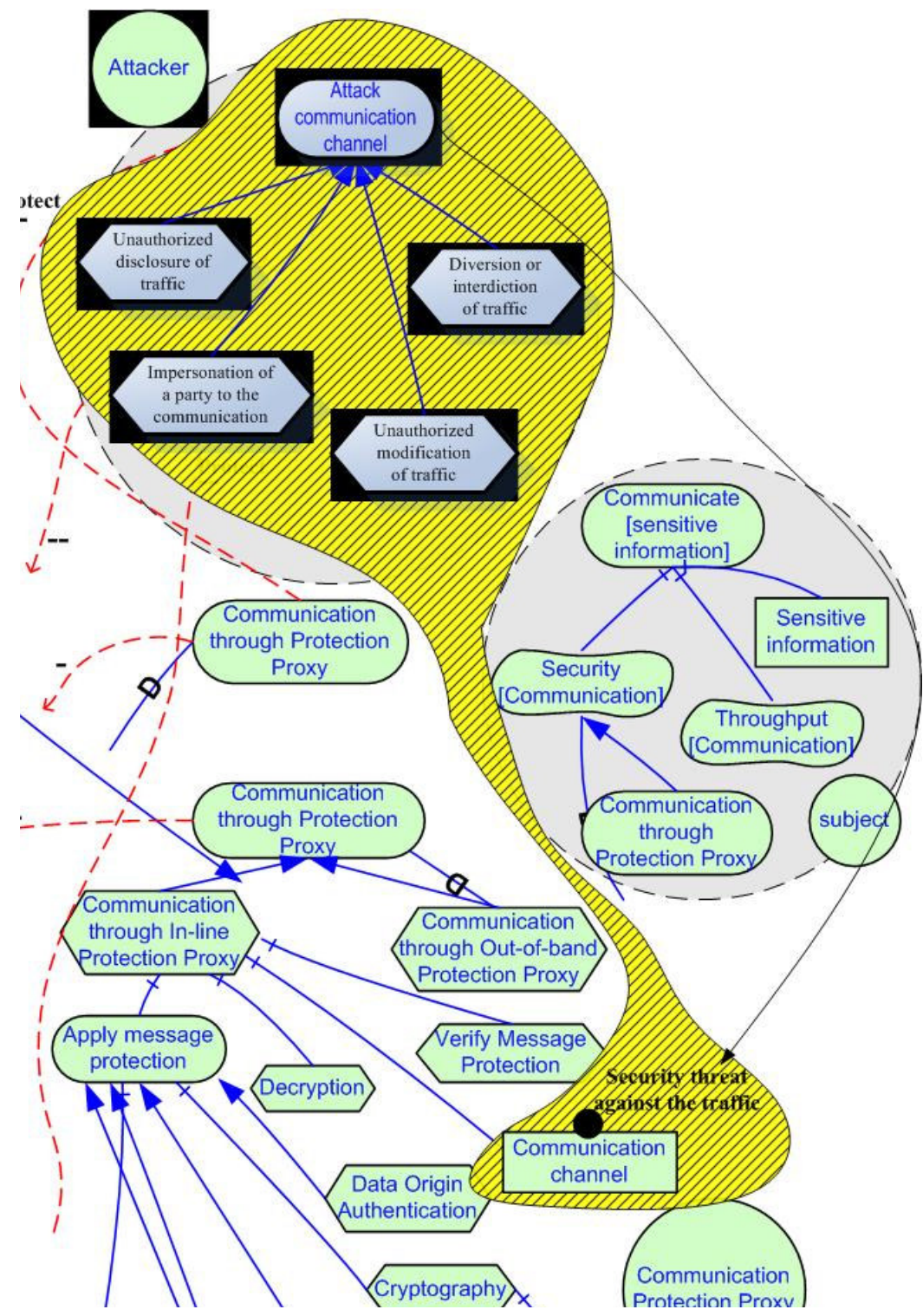

Figure 6-7. A unit of knowledge from the whole knowledge chunk of Figure 6-5, which corresponds to the knowledge unit of Figure 5-5-B

The algorithm that extracts this unit of knowledge (Figure 5-5-B) from the knowledge chunk works as below:

- $\quad$ Locate the vulnerability which the query refers to 
- Extract the element (resource or goal) that the vulnerability is assigned to

- Extract all malicious goals which are linked to the vulnerability

- Extract all tasks or alternative attacks under obtained malicious goals

\subsection{Lessons Learnt and Future Works}

We discussed how two different formats of knowledge: text based knowledge as an amorphous unstructured body of knowledge, and design patterns as a semi-formal structured chunk of knowledge can be transformed to the proposed KB models. The transformation is based on human judgment and interpretation, which affects the resulting models. As a result, it is likely to have two different knowledge models from the same knowledge source that are developed by different transformers or by the same transformer in different times. In addition, the organization and presentation of the knowledge in the source is reflected in the resulting knowledge models.

We briefly discussed the algorithms to retrieve a sub-graph (knowledge unit) from the large chunk of knowledge. However, a question is remained unanswered that how designers may use the resulting models. Is it more useful and efficient to present the knowledge in a large and complicated chunk of goal model? Or dealing with numerous but simple units of knowledge which are extracted from the large chunk is more practical and efficient? Answering these questions requires further empirical studies involving real-world practitioners.

We concluded that transforming and representing security knowledge in the goal model format is beneficial in several dimensions: Transforming knowledge from a source to goal oriented knowledge models may lead to discovering incompleteness, insufficiency, and inconsistencies in the source knowledge. The goal model 
representation of knowledge of design patterns provides the means to evaluate usefulness and suitability of the pattern in the context of the problem by taking advantage of goal model evaluation, which was illustrated in the Chapter four in a number of case studies. In this way, the designer examines if the intents of the pattern, which is expressed as a high-level goal in the goal model, is satisfied. An integrated goal model of multiple design patterns can express the trade-offs among different design patterns, and goal model evaluation helps the designer decide among alternative patterns as well.

The question which arises in this context is that do different structures of the same knowledge content bear different semantics? Does the goal-oriented approach for representing knowledge lead to a different interpretation of knowledge? Does the goaloriented approach for representing knowledge provide stronger semantic than the ordinary text-based parent representation? These concerns would be studied in future empirical studies. In addition, this study was limited to transforming two knowledge sources to the proposed knowledge model, and in future works, we aim to examine transforming more diverse knowledge sources to the proposed knowledge models. 


\section{Contributions, Conclusions, Limitation, and Future Works}

\subsection{Contributions and Conclusions}

In this work, we began by considering the criteria for a conceptual modeling technique that enables designers to model and analyze security trade-offs among competing goals of multiple actors to achieve a good-enough security. We studied existing approaches to trade-off analysis, and identified limitations of these approaches. The main contribution of this work is a goal-oriented approach for modeling security aspects such as threats, vulnerabilities, assets, security goals and trade-offs they impose to other goals of multiple actors. The notation is proposed based on the discussed criteria and limitation of previous works. The modeling notation is accompanied with a qualitative trade-off analysis and decision making procedure which is based on goal model evaluation. The procedure provides the designers with an assessment of security mechanisms' impact on actors' goals and threats.

To model and analyze security trade-offs the designer needs a source of security knowledge to support the decision making process. The other main contribution of this work is a software security trade-offs Knowledge Base from which the designer can make inquiries about potential threats, attacks, and vulnerability points in a context, as well as existing security solutions and their impact on threats and goals of actors. We discussed the practical issues regarding to populating the $\mathrm{KB}$ from various knowledge sources, and we described problems of transforming different knowledge format to the $\mathrm{i}^{*}$ knowledge models. 
Explicit representing security requirements and security knowledge in form of goal models provides a proper basis for analyzing consequences of applying alternative security mechanisms and visually expressing the concepts of trade-offs in the goal models of the system. This guides the designer to make the trade-offs based on an informed judgment, while other design concerns such as level of performance and usability are still considered and incorporated in decision makings.

The main conclusions and contributions of this work are listed and described as below:

$\rightarrow$ Criteria for a conceptual modeling technique for security trade-off analysis: We began by considering the criteria of a proper conceptual modeling technique for dealing with security trade-offs. We concluded that a conceptual modeling technique that expresses the security trade-offs and enables security trade-off analysis should model three kinds of concepts: i) Goals, ii) Actors and iii) Security specific concepts.

$\rightarrow$ Main advantages and disadvantages of existing approaches: We studied existing approaches to trade-off analysis, and identified limitations of these approaches. ATAM and CBAM are two general purpose architectural trade-off analysis methods which do not explicitly express and model security trade-offs. STVD and AORDD propose quantitative approaches for analyzing trade-offs by taking advantage of BBN. However, inaccuracy and unavailability of quantitative measures are among the limitations of such approaches. Goal and agent oriented approaches such as secure 
Tropos provide a suitable basis for trade-off analysis. However, existing goal oriented approaches don not express security trade-offs explicitly.

$\rightarrow$ A goal oriented notation for modeling security trade-offs: The main contribution of this work is a goal oriented notation for modeling security trade-offs and other security aspects such as threats, vulnerabilities, assets, and security goals. We propose a meta-model of security concepts which covers the mentioned concepts. Taking advantage of its goal and agent orientation, we have added extensions to the $\mathrm{i}^{*}$ modeling notation for expressing security concepts such as attacks and vulnerability points. Table 7-1 summarizes properties of the proposed method regarding the criteria of a proper conceptual modeling technique for modeling and analyzing security trade-offs.

Table 7-1. Summary of properties of proposed approach according to the criteria of conceptual modeling technique

\begin{tabular}{|l|l|}
\hline Method Requirements & Suggested approach \\
\hline Goals & Modeled using goals and softgoals elements of i* \\
\hline Relations of goals & $\begin{array}{l}\text { Modeled using i* goal dependency modeling. } \\
\text { Competition and trade-offs are modeled by contribution } \\
\text { links and relation between attacks and goals. }\end{array}$ \\
\hline $\begin{array}{l}\text { Extents and measures of } \\
\text { goals }\end{array}$ & $\begin{array}{l}\text { Modeled qualitatively by contribution links of type -, - -, } \\
+,++\end{array}$ \\
\hline $\begin{array}{l}\text { Inaccurate or incomplete } \\
\text { knowledge }\end{array}$ & $\begin{array}{l}\text { Modeled by unknown contribution links, and goal model } \\
\text { evaluation propagates them to related elements }\end{array}$ \\
\hline $\begin{array}{l}\text { Goals contribution } \\
\text { structure }\end{array}$ & $\begin{array}{l}\text { Structured by sub-goals, task decomposition, } \\
\text { contribution links }\end{array}$ \\
\hline Multiple actors & $\begin{array}{l}\text { Multiple malicious and non malicious actors can be } \\
\text { modeled }\end{array}$ \\
\hline $\begin{array}{l}\text { Trade-off within a single } \\
\text { actor or across actors }\end{array}$ & $\begin{array}{l}\text { Trade-off within a single actor or across actors can be } \\
\text { modeled }\end{array}$ \\
\hline $\begin{array}{l}\text { Security specific trade-off } \\
\text { concepts }\end{array}$ & $\begin{array}{l}\text { Modeled by security extensions to i* notation derived } \\
\text { from the meta-model }\end{array}$ \\
\hline Trade-off analysis method & $\begin{array}{l}\text { Security goal model evaluation technique supports } \\
\text { qualitative trade-off analysis }\end{array}$ \\
\hline
\end{tabular}

$\rightarrow$ A security trade-off analysis procedure: The trade-off analysis procedure, which is based by on goal model evaluation, evaluates consequences of alternatives 
security mechanisms on actors' goals and threats. Goal model evaluation is the procedure to ensure that actors' top level goals are satisfied by the choices they have made. The evaluation starts with the assumption that the attacker would be successful in performing the malicious tasks, and then the rest of the procedure examines which goals and softgoals of other actors are partially or fully satisfied (denied) for each alternative security design solution.

$\rightarrow$ Three case studies of modeling and analyzing trade-offs: The proposed modeling notation and analysis technique are illustrated through three example cases which were previously studied in similar works. The comparison of resulting models reveals that the proposed modeling notation explicitly and visually expresses security trade-offs among various security mechanisms and alternative solutions. This approach results in models which contain advantages and disadvantages of each alternative, conflicts, trade-offs and impacts of each decision.

$\rightarrow$ Properties of software security knowledge sources: We surveyed various security knowledge sources considering and comparing properties and limitations of each one to extract properties of a desired software security trade-offs KB. We have identified four major categories of software security knowledge sources: security textual knowledge, security experiences' documents, security design patterns, and structured catalogues and knowledge bases. We discussed properties of each knowledge structure such as its knowledge items, generality, formality, domain independence, and units of knowledge.

$\rightarrow$ A preliminary schema for a software security trade-offs knowledge base:

Another main contribution of this work is a software security trade-offs knowledge base 
to catalogue security knowledge such a common threats and their corresponding security mechanisms, NFR goals (besides security goals) and the contributions of security decisions to the other goals. The schema of the $\mathrm{KB}$ is similar to the meta-model of the proposed notation to cover required elements which the designer needs to incorporate into the trade-off models, and the knowledge in the $\mathrm{KB}$ is codified using the proposed notation.

$\rightarrow$ Unit of knowledge of the proposed knowledge base schema: We proposed various reusable and meaningful combinations of the elements of the KB schema as the knowledge units. The structure and combination of elements in the unit of knowledge depends on the usage of knowledge, navigation path through the body of knowledge, and type of the questions which the designer inquires from the KB to make security tradeoffs. We suggested algorithms for retrieving a sub-graph of the knowledge chunk for extracting a knowledge unit, based the question that the designer inquiries from the KB.

$\rightarrow$ Populating the knowledge base (two samples): Populating the KB requires a human transformer review the source knowledge, interpret and elicit the knowledge, and transform the knowledge into the proposed $\mathrm{KB}$ schema. We transformed software security knowledge from two knowledge structures: parts of a book chapter and a security pattern. The results of these case studies revealed that transforming knowledge from a source to goal oriented knowledge models may lead to discovering incompleteness, insufficiency, and inconsistencies in the source knowledge. The human transformer may incorporate some parts of the knowledge source directly to the target knowledge format, or may need to interpret or change some parts of the sources knowledge, or add extra elements to the final knowledge models. Finally, the goal model 
representation of knowledge provides the means to evaluate usefulness and suitability of knowledge in the context of the problem.

\subsection{Limitation and Future Works}

This work introduces a technique for modeling and analyzing security trade-offs in a multi-agent environment. In the proposed technique, designers incorporate common threats and vulnerabilities into the goal model, add impact of threats and alternative solutions on goals of actors, and examine the risk of attacks and success of corresponding security solutions by goal model evaluation. In this process, designers may rely on security knowledge gained from various knowledge sources including the proposed KB. However, the proposed framework needs to be organized in a methodological framework which provides the designer with a step by step process for modeling activities, analysis procedure, and knowledge acquisition. In future work, we aim to adapt the trade-off analysis methodological framework for integration into everyday design practice.

Although the $\mathrm{i}^{*}$ notation provides the proper basis for modeling and analyzing trade-offs, the models become large and complex quickly, and scalability issues have become a barrier for understandability and efficiency of the models. This work is limited in some other aspects, and needs further follow up works such as including risk analysis approaches, evaluating of the proposed techniques and knowledge base, and required empirical studies, evidences, and evaluations. 


\subsubsection{Further Case Studies for Evaluating the Modeling Notation and Analysis Method}

First of all, the case studies which exemplify the modeling notation and decision making procedure are limited to three case studies, which does not provide strong and enough evaluation on weaknesses and limitations of the proposed method. Further case studies need to be done to evaluate the expressiveness, completeness and sufficiency of the proposed modeling notation to express security trade-offs.

The usefulness, expressiveness and practicality of the proposed notation need to be evaluated by detail case studies and empirical experiments. In addition, further studies need to be performed to evaluate the correctness of the decisions that designer make by employing the proposed modeling and analyzing technique.

\subsubsection{The Need for further Empirical Studies on the Proposed Trade-off Analysis Framework}

In future work, we aim to conduct empirical studies such as interviews, ethnographies, and surveys to investigate how security designers make trade-offs in current practice, and what conceptual modeling technique may facilitate dealing with security trade-offs. Moreover, usefulness, expressiveness, comprehensiveness, and understandability of the proposed notation and analysis method needs to be evaluated by empirical studies involving practitioners who will be asked to develop models using the proposed framework, and developers who will be asked to interpret and describe models which are developed by the proposed notation. It is left to future works to study current practices for dealing with security trade-offs which are based on evaluating the 
compliance of security requirements with standards and checklists after the final decisions on system design is made.

\subsubsection{Further Studies on the Proposed KB}

Future empirical studies are needed to investigate what knowledge sources are used by software designers and Information Systems professionals while probably they are not security experts, how they use knowledge sources such as security standards, guidelines, textbooks, and previous experiences, and how the proposed KB may be employed. In general, the claims within this work could benefit by the design and execution of empirical studies to test the usefulness, comprehensiveness, and practicality of the proposed $\mathrm{KB}$ schema and the graphical knowledge models. This work could be strengthened by empirical evidences which specify what knowledge type of format such as text, graphical models, semi-structure text, etc. is useful, understandable, and practical in fieldwork. This study needs to be continued to investigate how practitioners may browse and use the proposed knowledge models, and do they prefer numerous small units of knowledge or one large complicated chunk.

We exemplified transforming knowledge and populating the $\mathrm{KB}$ through two cases. However, in order to assess the expressiveness and comprehensiveness of the $\mathrm{KB}$ schema and the graphical knowledge codifying notation, a wider variety of knowledge formats should be transformed to the proposed $\mathrm{KB}$ schema. It is left to future studies to investigate if different structures of the same knowledge content bear different semantics? Does the goal-oriented approach for representing knowledge lead to a different interpretation of knowledge? Does the goal-oriented approach for representing knowledge provide stronger semantic than the ordinary text-based parent representation? 
Finally, further research is required to study how additional knowledge items should be systematically elicited different knowledge sources to cover the incompleteness of one source. 


\section{References}

[1] IBM Internet Security Systems X-Force® 2006 Trend Statistics, Available at http://www.iss.net/documents/whitepapers/X_Force_Exec_Brief.pdf, IBM Internet Security Systems Executive Brief, January 2007

[2] Sandhu, R., Good-Enough Security: Toward a Pragmatic Business-Driven Discipline," IEEE Internet Computing, Vol. 07, No. 1, (2003) 66-68

[3] Adams, A., Sasse, M. A., Users are not the enemy, Communications of the ACM, v.42 n.12 (1999) 40-46

[4] Sasse, M. A., Computer Security: Anatomy of a Usability Disaster, and a Plan for Recovery. CHI (2003)

[5] DeWitt, A. J., Kuljis, J., Aligning Usability And Security-A Usability Study Of Polaris, In Proceedings of the 2006 Symposium On Usable Privacy and Security (2006)

[6] Houmb, S. H., Georg, G., Jürjens, J., France, R., An Integrated Security Verification and Security Solution Design Trade-off Analysis, In Integrating Security and Software Engineering: Advances and Future Visions, IDEA Group Publishing (2007) $190-219$

[7] Dieste, O., Juristo, N., Moreno, A. M., Pazos, J., Sierra, A., Conceptual Modelling in Software Engineering and Knowledge Engineering: Concepts, Techniques and Trends, Handbook of Software Engineering and Knowledge Engineering, Vol. 1, No. $1(2000)$ 
[8] Mylopoulos, J., Borgida, A., Yu, E., Representing software engineering knowledge, Automated Software Engineering, no. 4 (1997)

[9] McDermott, J., Fox, C., Using Abuse Case Models for Security Requirements Analysis, In Proceedings 15th. IEEE Annual Computer Security Applications Conference (1999) 55-64

[10] Jürjens, J., Secure Systems Development with UML, Springer Academic Publishers, Germany (2004)

[11] Moffett, J. D., Haley, C. B., Nuseibeh, B., Core Security Requirements Artefacts, Department of Computing, The Open University, Milton Keynes UK, Technical Report 2004/23 (2004)

[12] Haley, C. B., Moffett, J. D., Laney, R., Nuseibeh, B., A framework for security requirements engineering. In: Software Engineering for Secure Systems Workshop (SESS'06), co-located with the 28th International Conference on Software Engineering (ICSE'06) (2006) 35 - 42

[13] Lodderstedt. T., Basin. D., Doser. J., SecureUML: A UML-Based Modeling Language for Model-Driven Security, In Proceedings of the 5th International Conference on The Unified Modeling Language, Springer LNCS 2460 (2002) 426-441

[14] Houmb. S. H., Braber. F. D., Lund. M. S., Stølen. K., Towards a UML Profile for Model-Based Risk Assessment, In Proc. UML'2002 Satellite Workshop on Critical Systems Development with UML, (200) 79-91

[15] Johnson, P., Lagerstrom, R., Norman, P., Simonsson, M., Extended Influence Diagrams for Enterprise Architecture Analysis, In: Enterprise Distributed Object Computing Conference, EDOC '06. 10th IEEE International (2006) 3-12 
[16] Mayer, N., Rifaut, A., Dubois, E., Towards a Risk-Based Security Requirements Engineering Framework, 11th International Workshop on Requirements Engineering: Foundation for Software Quality (REFSQ'05), in conjunction with CAiSE'05, (2005)

[17] Dardenne, A., van Lamsweerde, A., Fickas, S., Goal-Directed Requirements Acquisition, in The Science of Computer Programming 20 (1993) 3-50

[18] Chung, L., Nixon, B. A., Yu, E., Mylopoulos, J., Non-Functional Requirements in Software Engineering, Kluwer Academic Publishing (2000)

[19] Yu, E., Modeling Strategic Relationships for Process Reengineering, PhD thesis, Department of Computer Science, University of Toronto, Canada (1995)

[20] Castro, J., Kolp, M., Mylopoulos, J., A requirements-driven development methodology, In Proc. of the 13th Int. Conf. on Advanced Information Systems Engineering, CAiSE'01 (2001) 108-123

[21] Liu, L., Yu, E., Mylopoulos, J., Analyzing Security Requirements as Relationships among Strategic Actors, In 2nd Symposium on Requirements Engineering for Information Security (SREIS) (2002)

[22] Liu, L., Yu, E., Mylopoulos, J., Security and Privacy Requirements Analysis within a Social Setting. In IEEE Joint International Conference on Requirements Engineering (2003) 151-161

[23] Giorgini, P., Massacci, F., Mylopoulos, J., Zannone, N., Modeling Security Requirements through Ownership, Permission and Delegation. In: 13th IEEE International Requirements Engineering Conference (2005) 167-176 
[22] Liu, L., Yu, E., Mylopoulos, J., Security and Privacy Requirements Analysis within a Social Setting. In IEEE Joint International Conference on Requirements Engineering (2003) 151-161

[23] Giorgini, P., Massacci, F., Mylopoulos, J., Zannone, N., Modeling Security Requirements through Ownership, Permission and Delegation. In: 13th IEEE International Requirements Engineering Conference (2005) 167-176

[24] Bresciani, P., Giorgini, P., Mouratidis, H., On Security Requirements Analysis for Multi-Agent Systems, Proceedings of 2nd International Workshop on Software Engineering for Large-Scale Multi-Agent Systems SELMAS, in conjunction with the 25th International Conference on Software Engineering (2003)

[25] Mouratidis, H., Giorgini, P., Manso, G., Philp, I, A Natural Extension of Tropos Methodology for Modelling Security, In Proceedings of the Workshop on Agentoriented methodologies, at OOPSLA (2002) 91-103

[26] Mouratidis, H., Giorgini, P., Manso, Modelling Secure Multiagent Systems, in the Proceedings of the 2nd International Joint Conference on Autonomous Agents and Multiagent Systems (2003) 859 - 866

[27] Bass, L., Clements, P., Kazman, R., Software Architecture in Practice, Second Edition, Addison Wesley (2003)

[28] Houmb, S. H., G. Georg, G., The Aspect-Oriented Risk-Driven Development (AORDD) Framework, In Proc. of the Int. Conf. on Software Development (SWDC.REX) (2005) 81-91

[29] Susi, A., Perini, A., Mylopoulos, J., The Tropos Metamodel and its Use, Informatica No. 29 (2005) 401-408 
[30] Grance, T., Stevens, M., Myers, M., Guide to Selecting Information Technology Security Products, Recommendations of the National Institute of Standards and Technology, NIST Special Publication 800-36 (2003)

[31] US-CERT Vulnerability Notes Database, United States Computer Emergency Readiness Team, http://www.kb.cert.org/vuls

[32] Anderson, R., Security Engineering: a guide to Building dependable Distributed systems, John Wiley and Sons (2001)

[33] Pfleeger, C. P., Pfleeger,S. L., Security in Computing, Prentice Hall, 3dr edition (2002)

[34] Horkoff, J., Using i* Models for Evaluation, Masters Thesis, University of Toronto, Department of Computer Science (2006)

[35] Lindland, O., Sindre, G., Solvberg, A., Understanding quality in conceptual modeling, Software, IEEE 11 (1994) $42-49$

[36] Nancy, R., Mead, Gary McGraw, "A Portal for Software Security" (with Gary McGraw), IEEE Security \& Privacy, 2(4), 2005, pp. 75-79

[37] Barnum, S., McGraw, G., Knowledge for Software Security, IEEE Security \& Privacy 3(2): 74-78 (2005)

[38] Laverdière, M-A., Mourad, A., Hanna, A., Debbabi, M., Security Design Patterns: Survey and Evaluation. In the Proceedings of the IEEE Canadian Conference on Electrical and Computer Engineering, CCECE'2006, May 7-10, 2006, Ottawa, Ontario, Canada, IEEE Press

[39] SANS Top-20 Internet Security Attack Targets (2006 Annual Update), Available at http://www.sans.org/top20/ (2006) 
[40] Elahi, G., Yu, E., A Goal Oriented Approach for Modeling and Analyzing Security Trade-Offs, To be appeared in Proceeding of 26th International Conference on Conceptual Modeling (ER2007), Auckland, New Zealand, November 2007

[41] Protect the Network Core From Internal Threats and Malware, by Mazu Networks, Available at http://cramsession.bitpipe.com/detail/RES/1185896904_326.html (2007)

[42] Blakley, B., Heath. C., Security Design Patterns, Technical Guide, Available at http://macedonia.uom.gr/ achat/articles/sec_patterns.pdf, The Open Group (2004)

[43] Gross, D., Yu. E., From Non-Functional Requirements to Design through Patterns, Requirements Engineering Journal, Vol 6, No. 1, 2001, pp. 18-36

[44] Mouratidis, H., P. Giorgini, M. Schumacher., Security Patterns for Agent Systems, In Proccedings of Eighth European Conference on Pattern Languages of Programs 25- 29, June 2003

[45] US-CERT Vulnerability Notes Database, United States Computer Emergency Readiness Team, http://www.kb.cert.org/vuls

[46] Tracy, M., Jansen, W., Scarfone. K., Butterfield. J., Guidelines on Electronic Mail Security, Recommendations of the National Institute of Standards and Technology, NIST Special Publication 800-45 (2007)

[47] Regli, W.C., Hu, X., Atwood, M., Sun, W., A Survey of Design Rationale Systems: Approaches, Representation, Capture and Retrieval

[48] Helm. R., Johnson. R., Vlissides. J., Design Patterns: Elements of Reusable Object-Oriented Software, Erich Gamma, Addison-Wesley, October 1994, ISBN: 0-201$63361-2$ 
[49] Alexander, I., Initial Industrial Experience of Misuse Cases in Trade-Off Analysis, In Proceedings of the 10th Anniversary IEEE Joint International Conference on Requirements Engineering (2002) 61-70

[50] Sindre, G., Opdahl, L., Eliciting security requirements by misuse cases, Proceedings of TOOLS Pacific, (2000) 120-131 